J. Noncommut. Geom. 6 (2012), 481-538

DOI $10.4171 / \mathrm{JNCG} / 98$
Journal of Noncommutative Geometry

(C) European Mathematical Society

\title{
A categorical approach to cyclic duality
}

\author{
Gabriella Böhm and Dragoş Ştefan
}

\begin{abstract}
The aim of this paper is to provide a unifying categorical framework for the many examples of para-(co)cyclic modules arising from Hopf cyclic theory. Functoriality of the coefficients is immediate in this approach. A functor corresponding to Connes's cyclic duality is constructed. Our methods allow, in particular, to extend Hopf cyclic theory to (Hopf) bialgebroids.
\end{abstract}

Mathematics Subject Classification (2010). 16E40, 18G30, 16W30.

Keywords. Para-(co)cyclic object, cyclic duality, (Hopf) bialgebroid, (co)monads, distributive laws and their (co)algebras.

\section{Contents}

1 $\Phi$-module functors and their morphisms $\ldots \ldots \ldots$. . . . . . . . . . . . . 485

2 Para-cocyclic objects and $\Phi$-module functors . . . . . . . . . . . . . . . . . . . . . 491

3 Examples from Hopf cyclic theory . . . . . . . . . . . . . . . . . . . 495

4 Para-cyclic objects . . . . . . . . . . . . . . . . . . . . . . . . . 504

5 Examples from Hopf cyclic theory . . . . . . . . . . . . . . . . . . 508

6 The cyclic duality functor . . . . . . . . . . . . . . . . . . 516

7 Back to the examples . . . . . . . . . . . . . . . . . . . 528

A Appendix: Modules, comodules and contramodules of Hopf algebroids . . . . . . . 531

References . . . . . . . . . . . . . . . . . . . 537

\section{Introduction}

Cyclic cohomology extends and unifies cohomology theories like de Rham cohomology and Lie algebra cohomology of matrices. It has applications e.g. in homological algebra, algebraic topology, Lie algebras, algebraic K-theory and so non-commutative differential geometry.

The foot-stone in cyclic cohomology theory is a so called cocyclic object, i.e., a cosimplicial object equipped with an isomorphism at each grade $n$, roughly implementing a cyclic permutation of the coface and codegeneracy morphisms. In particular, the $(n+1)$ st power of this cocyclic morphism is required to be the identity. The study of cocyclic objects, or a quest of their examples, can be divided to two 
steps. First one can deal with a more general structure, called a para-cocyclic object, obtained by relaxing the requirement about the $(n+1)$ st power of the cocyclic morphism at grade $n$ to be trivial. Truly cocyclic subobjects or quotients of para-cocyclic objects are then studied as a subsequent step.

There are many known examples of (para-)cocyclic objects, relevant for various purposes. A large family of examples, occurring as symmetries in non-commutative differential geometry, is associated to (co)module (co)algebras of bialgebras. The first example of this kind appeared in [9] where it was used by Connes and Moscovici to give a geometrical interpretation of the non-commutative Chern-character. Further examples of para-cocyclic objects, associated to (co)module (co)algebras of Hopf algebras were constructed by Hajac et al. in [12], where also non-trivial coefficients provided by (co)modules of the Hopf algebra were introduced. As a most important achievement, also criteria (on the coefficients) for the existence of truly cocyclic subobjects and quotients were found. These constructions were extended to bialgebras (extending Hopf algebras) by Kaygun in [16] and [17]. A new type of coefficients, so-called contramodules, was proposed by Brzeziński in [5]. In this way, currently there are known eight families of para-cocyclic objects associated to bialgebras: A cosimplicial object can be constructed from a module algebra or a comodule algebra, or from a module coalgebra or a comodule coalgebra $A$ (yielding four possibilities), using either a functor of the form $A \otimes(-)$ or a functor of the form $\operatorname{Hom}(A,-)$ (doubling the number of examples). In each case there turns out to be an appropriate choice of the coefficients resulting in a para-cocyclic structure.

Dually to (para-)cocyclic objects, one may consider (para-)cyclic objects, i.e., (para-) cocyclic objects in the opposite category. Using bialgebras, there can be constructed again eight families of examples.

As it was observed by Connes in [8], the category of cyclic objects and the category of cocyclic objects in a given category are isomorphic. This isomorphism, called cyclic duality, is not known to extend to the categories of para-cyclic and paracocyclic objects only to their appropriate subcategories. These (full) subcategories have those objects whose para-(co)cyclic morphisms are isomorphisms at each grade, cf. Khalkhali and Rangipour's work [18].

For para-(co)cyclic objects associated to (co)module (co)algebras of bialgebras, the para-(co)cyclic morphisms are not isomorphisms in general. They are isomorphisms, however, if the bialgebra in question is a Hopf algebra with an invertible antipode. In this case the eight families of associated para-cocyclic objects and the eight families of para-cyclic objects turn out to be pairwise related by cyclic duality.

The aim of this paper is to provide a general construction of para-(co)cyclic objects, including in particular existing constructions in terms of bialgebras together with their generalizations to bialgebroids, cf. [10]. We do not investigate here, however, the existence of truly (co)cyclic subobjects or quotients.

An important antecedent work of similar aims is Kaygun's paper [17], where a universal construction of para-(co)cyclic objects, including examples from bialgebras, 
was presented. The construction in this work is built on monoids and comonoids in symmetric monoidal categories. Therefore, while it is perfectly suitable to describe (co)module (co)algebras of bialgebras, it has to be generalized in order to be able to cope with bialgebroids over non-commutative base algebras. Such a generalization (under the names admissible septuple and its transposition map) was proposed in our previous work [4]. In Section 2 of the current paper we introduce a category in which admissible septuples and their transposition maps are special objects. This newly introduced category $\mathcal{A}$ comes equipped with a functor $Z^{*}$ from $\mathcal{A}$ to the category of para-cocyclic objects in the category of functors. As a consequence, any object in $\mathcal{A}$ induces a functor from a category (of coefficients) to the category of para-(co)cyclic objects in another category (usually the category of modules over a commutative ring). Thus the resulting construction of para-(co)cyclic modules is functorial for the choice of coefficients.

Behind the construction of the above category $\mathcal{A}$ there are some 2-categorical considerations. Consider an abstract 2-category $\mathcal{T}$ with a single 0 -cell $o$, a 1-cell $t: o \rightarrow o$ and 2-cells $\eta: o \Rightarrow t$ and $\mu: t t \Rightarrow t$, such that $\mu \circ \eta t=t=\mu \circ$ $t \eta$ and $\mu \circ \mu t=\mu \circ t \mu$ (i.e., such that $(t, \mu, \eta)$ is a monad in $\mathcal{T}$ ). A monad $(T, m, u)$ on a category $\mathcal{M}$ can be described then as a 2 -functor $F$ from $\mathcal{T}$ to an appropriate 2-category Cat of (some) categories, functors and natural transformations, such that $F o=\mathcal{M}, F t=T, F \mu=m$ and $F \eta=u$. A lax natural transformation between 2-functors $F, F^{\prime}: \mathcal{T} \rightarrow$ Cat is precisely the same as a monad morphism $(F t, F \mu, F \eta) \rightarrow\left(F^{\prime} t, F^{\prime} \mu, F^{\prime} \eta\right)$ in the sense of [23] (for a review see Section 1 below). Extending this picture, we may consider an abstract 2-category $S$ of three 0 cells $o, d$ and $c$, with two monads on $o$ related by a distributive law $\phi$, together with a so-called $S(\phi, c)$-algebra in $S(o, c)$ and a $S(d, \phi)$-algebra in $S(d, o)$. (For a review of algebras over distributive laws in [7] see Section 1 below.) The objects in our category $\mathcal{A}$ are 2-functors $S \rightarrow$ Cat and the morphisms are lax natural transformations between them. In Section 3 we collect eight families of examples of objects in $\mathcal{A}$ associated to bialgebroids.

A dual construction in Section 4 provides us with another category $\mathscr{B}$, admitting a functor $Z_{*}$ from $\mathscr{B}$ to the category of para-cyclic objects in the category of functors. In Section 5 we construct eight families of examples of objects in $\mathcal{B}$ in terms of bialgebroids.

In Section 6 we investigate the natural question how Connes' cyclic duality functor (more precisely its extension ${ }^{-}$to the categories of para-(co)cyclic objects with invertible para-(co)cyclic morphisms at each grade) lifts to a functor ${ }^{\Delta}$ between appropriate subcategories of $\mathcal{A}$ and $\mathcal{B}$, such that $Z_{*} \circ^{\triangle} \cong{ }^{\triangle} \circ Z^{*}$. Behind this lifting there are liftings of (co)monads.

In Section 7 we show that the examples associated to (co)module (co)algebras of a Hopf algebroid with a bijective antipode, belong to the subcategories of $\mathcal{A}$ and $\mathscr{B}$ on which the lifted cyclic duality functor ${ }^{\triangle}$ is defined. We also check that these examples are pairwise related by the functor ${ }^{\triangle}$. 
The paper contains an Appendix, summarizing some facts about modules, comodules and contramodules of bialgebroids and Hopf algebroids, used to construct the examples in the paper.

Notation. Composition of functors and the corresponding composition of natural transformations is denoted by juxtaposition. That is, for consecutive functors $F: \mathcal{C} \rightarrow \mathscr{D}$ and $G: \mathscr{D} \rightarrow \mathcal{E}$, the composite is denoted by $G F$, with object map $X \mapsto G F X$. For endofunctors $T: \bigodot \rightarrow \ell$, we also write $T T=T^{2}$. For natural transformations $v: F \rightarrow F^{\prime}$ and $w: G \rightarrow G^{\prime}$, the value of $v$ at an object $X$ is denoted by $v X$. Moreover, $G v$ is a natural transformation $G F \rightarrow G F^{\prime}$ whose value at $X$ is given by $G(v X)$ - written simply as $G v X$. Similarly, $w F$ is a natural transformation $G F \rightarrow G^{\prime} F$, with value at $X$ given by $w(F X)=w F X$. The composition of consecutive natural transformations is denoted by o. The identity morphism of any object $X$ is denoted by the same symbol $X$. In order to simplify some computations, we shall use the string representation of functors and their natural transformations. Throughout this paper, the composition of functors is represented by horizontal juxtaposition of strings, the functor acting first being represented by the rightmost string. A natural transformation $F_{1} \ldots F_{n} \rightarrow G_{1} \ldots G_{m}$ will be represented as a 'microchip' with $n$ inputs $F_{1}, \ldots, F_{n}$ at its top and $m$ outputs $G_{1}, \ldots, G_{m}$ at its bottom. The identity natural transformation of a functor $F$ will be represented just as a string, without any box, as in the first diagram. For a natural transformation $v: F \rightarrow F^{\prime}$, we draw $G v: G F \rightarrow G F^{\prime}$ and $v H: F H \rightarrow F^{\prime} H$ as in the second and the third pictures.
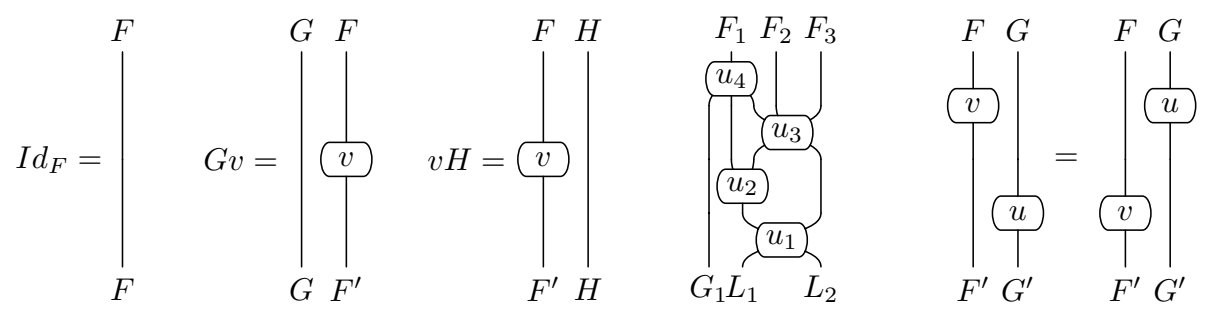

The composition of natural transformations is represented by vertical juxtaposition of the corresponding 'layers'. The natural transformation acting first is represented by the top layer. Hence if translating diagrams to formulae, we have to read our diagrams from the bottom to the top and from left to right: For example, let us consider natural transformations

$$
\begin{array}{ll}
u_{1}: K_{1} H_{2} \rightarrow L_{1} L_{2}, & u_{2}: G_{2} H_{1} \rightarrow K_{1}, \\
u_{3}: G_{3} F_{2} F_{3} \rightarrow H_{1} H_{2}, & u_{4}: F_{1} \rightarrow G_{1} G_{2} G_{3} .
\end{array}
$$

Then the fourth diagram of the above picture is the string representation of the natural transformation

$$
G_{1} u_{1} \circ G_{1} u_{2} H_{2} \circ G_{1} G_{2} u_{3} \circ u_{4} F_{2} F_{3} \text {. }
$$


In this notation, naturality of morphisms is visualized by their behaviour as 'pearls' on the strings. That is, those boxes which do not have common ingoing or outgoing strings, can be freely moved above or below each other, cf. the last equality in the above figure.

The above diagrammatic notation is used more generally in any 2-category: 1cells are represented by vertical strings, their domains corresponding to the surfaces on their right and codomains corresponding to the surfaces on their left. 2-cells are represented by boxes, with domains represented by 'incoming legs' at their top and codomains represented by 'outgoing legs' at their bottom. Horizontal and vertical compositions in a 2-category are represented by horizontal and vertical juxtapositions of such diagrams. Diagrams like the rightmost one above, come from the middle four interchange law.

Note that in the literature the dual diagrammatic notation is used equally frequently. In that case, our strings representing 1-cells are replaced by the orthogonal lines - hence surfaces on the sides of the original lines are replaced by source and target points of the new orthogonal lines; and source and target points of the original lines are replaced by surfaces on the sides of the new orthogonal lines. In this notation 2-cells are represented by labels of the faces surrounded by their domain and codomain 1-cells. For instance the 2-cell in the second figure above is represented as

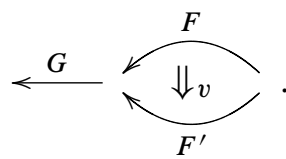

Though both diagrammatic notations contain precisely the same information, in this paper we prefer to work with string diagrams.

\section{1. $\Phi$-module functors and their morphisms}

In this section we recall some notions from category theory, the constructions of the later sections are built on.

The following notions are introduced in [23].

Definition 1.1. A monad on a category $\mathcal{M}$ is a triple $(T, m, u)$, where $T: \mathcal{M} \rightarrow \mathcal{M}$ is a functor and $m: T^{2} \rightarrow T$ and $u: \mathcal{M} \rightarrow T$ are natural transformations, called the multiplication and unit, respectively, which satisfy the last two (associativity and unitality) conditions in the following figure. (Our string representations for the multiplication and the unit of a monad are introduced in the first two equalities in the same figure.) 

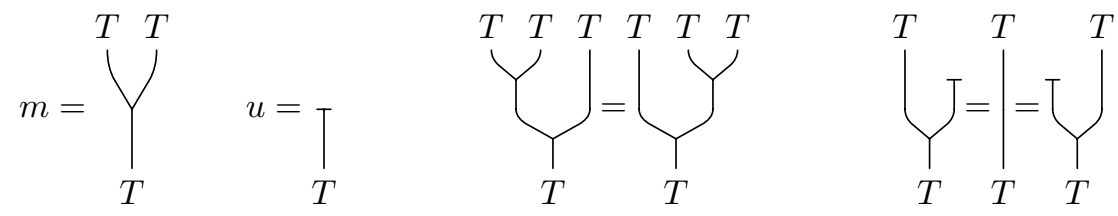

A morphism from a monad $\left(T^{\prime}, m^{\prime}, u^{\prime}\right)$ on $\mathcal{M}^{\prime}$ to a monad $(T, m, u)$ on $\mathcal{M}$ is a pair $(F, f)$, where $F: \mathcal{M}^{\prime} \rightarrow \mathcal{M}$ is a functor and $f: T F \rightarrow F T^{\prime}$ is a natural transformation which satisfies the following two relations.
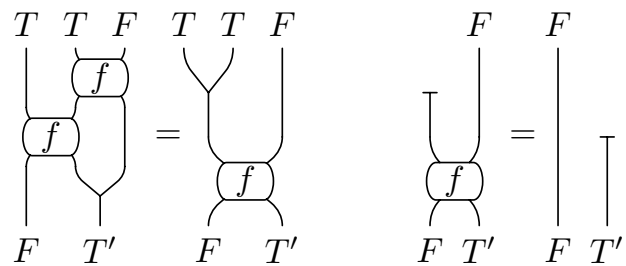

Monads and their morphisms constitute a category, with identity morphism $(\mathcal{M}, T):(T, m, u) \rightarrow(T, m, u)$ and composition law $(G, g) \circ(F, f)=(G F, G f \circ$ $g F)$, for monad morphisms $(F, f):\left(T^{\prime \prime}, m^{\prime \prime}, u^{\prime \prime}\right) \rightarrow\left(T^{\prime}, m^{\prime}, u^{\prime}\right)$ and $(G, g)$ : $\left(T^{\prime}, m^{\prime}, u^{\prime}\right) \rightarrow(T, m, u)$.

The category of monads and their morphisms is in fact isomorphic to a full subcategory in the category of 2-functors and lax natural transformations. Let us denote in any 2-category the horizontal composition by juxtaposition and the vertical composition by $\circ$. Consider the 2-category Cat whose 0 -cells are some categories (whose monads we are aiming to describe), 1-cells are functors and 2-cells are natural transformations. (In order to avoid set theoretical problems arising from the paradox of "the category of all categories", some restrictions on the 0-cells are needed. Since our most important examples in Sections 3 and 5 are (co)module categories, for our purposes allowing only for small categories would be too restrictive. All of our examples are included, for example, if 0-cells are all admissible or all locally presentable categories, cf. [21]. Readers interested in other examples might choose other classes (or in some cases even finite sets) of 0-cells to define an appropriate (large) 2-category Cat.) On the other hand, consider the 2-category freely generated by a monad. That is, the 2-category $\mathcal{T}$ with a single 0 -cell $o$, a non-identity 1-cell $t: o \rightarrow o$ and its iterated horizontal composites, and 2-cells given by composites of the non-identity 2-cells $\eta: o \Rightarrow t$ and $\mu: t t \Rightarrow t$, modulo the relations $\mu \circ \eta t=t=\mu \circ t \eta$ and $\mu \circ \mu t=\mu \circ t \mu$ (for a diagrammatic representation see Definition 1.1); that is, such that $(t, \mu, \eta)$ is a monad in $\mathcal{T}$. A 2 -functor $K: \mathcal{T} \rightarrow$ Cat is precisely the same as a $\operatorname{monad}(K t, K \mu, K \eta)$ on the category $K o$.

A lax natural transformation $K^{\prime} \rightarrow K$, for 2-functors $K, K^{\prime}: \smile \rightarrow \mathcal{D}$ between any 2-categories $\mathcal{C}$ and $\mathcal{D}$, is given by 1 -cells $F_{C}: K^{\prime} C \rightarrow K C$ in $\mathscr{D}$, labelled by the

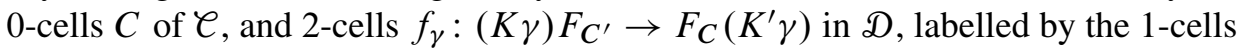
$\gamma: C^{\prime} \rightarrow C$ in $\mathcal{C}$. These data obey the following conditions. 
(i) Naturality of $f$; that is, for any 2-cell $\Gamma: \gamma \rightarrow \delta$ in $\mathcal{\ell}$, the diagram

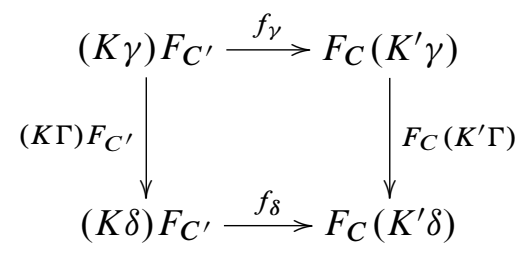

commutes.

(ii) Compatibility of $f$ with the horizontal composition; that is, for any 1-cells $C^{\prime \prime} \stackrel{\gamma^{\prime}}{\rightarrow} C^{\prime} \stackrel{\gamma}{\rightarrow} C$, the identity $f_{\gamma \gamma^{\prime}}=f_{\gamma}\left(K^{\prime} \gamma^{\prime}\right) \circ(K \gamma) f_{\gamma^{\prime}}$ holds.

(iii) Compatibility of $f$ with the identity 1-cells; i.e., for any identity 1-cell $C \stackrel{C}{\longrightarrow} C$, $f_{C}$ is equal to the identity 2-cell $F_{C} \stackrel{F_{C}}{\longrightarrow} F_{C}$.

A lax natural transformation between 2-functors $K, K^{\prime}: \mathcal{T} \rightarrow$ Cat is given then by a functor $F=F_{o}: K^{\prime} o \rightarrow K o$ and a natural transformation $f=f_{t}:(K t) F \rightarrow$ $F\left(K^{\prime} t\right)$. (The value of $f$ on the other 1-cells $t^{n}$ for $n \neq 1$ is determined by the compatibility conditions (ii) and (iii) with the horizontal composition and with the identity 1-cell.) The naturality condition (i), applied to $\Gamma=\mu$ and $\Gamma=\eta$, respectively, yields precisely the same conditions in Definition 1.1 on a monad morphism $(F, f):\left(K^{\prime} t, K^{\prime} \mu, K^{\prime} \eta\right) \rightarrow(K t, K \mu, K \eta)$.

Definition 1.2. An algebra for a monad $(T, m, u)$ on a category $\mathcal{M}$, is a monad morphism from the identity monad on the terminal category (of a single object and its identity morphism) to $(T, m, u)$, that is, a pair $(M, \varrho)$, where $M$ is an object in $\mathcal{M}$ and $\varrho: T M \rightarrow M$ is a morphism in $\mathcal{M}$ such that the first two diagrams in
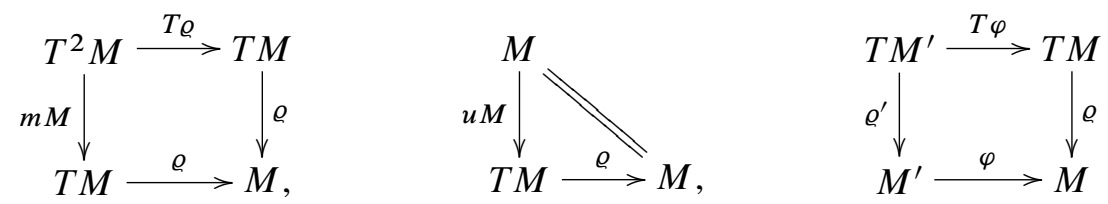

commute. A morphism of $T$-algebras $\left(M^{\prime}, \varrho^{\prime}\right) \rightarrow(M, \varrho)$ is a morphism $\varphi: M^{\prime} \rightarrow M$ in $\mathcal{M}$ such that the third diagram above commutes. Algebras of a monad $T$ and their morphisms constitute the so-called Eilenberg-Moore category $\mathcal{M}^{T}$.

Note that via composition on the right, a monad $T: \mathcal{M} \rightarrow \mathcal{M}$ induces a monad $\operatorname{Cat}(T,-)$ on the category $\operatorname{Cat}(\mathcal{M},-)$, whose objects are functors of domain $\mathcal{M}$ and whose morphisms are natural transformations. Symmetrically, there is a monad Cat $(-, T)$ acting by composition on the left on the category Cat $(-, \mathcal{M})$, whose objects are functors of codomain $\mathcal{M}$ and whose morphisms are natural transformations. In order to distinguish algebras of these induced monads from $T$-algebras, we call an 
algebra of the monad Cat $(T,-)$ a right $T$-module functor and we term an algebra of the monad Cat $(-, T)$ a left $T$-module functor.

Definition 1.3. Consider two monads $\left(T_{l}, m_{l}, u_{l}\right)$ and $\left(T_{r}, m_{r}, u_{r}\right)$ on the same category $\mathcal{M}$. A (monad) distributive law is a natural transformation $\Phi: T_{r} T_{l} \rightarrow T_{l} T_{r}$ such that the following four relations hold true.
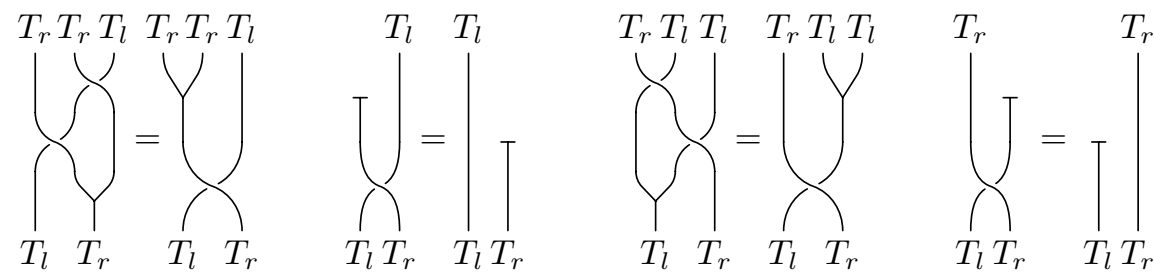

The first two defining relations are equivalent to the fact that $\left(T_{l}, \Phi\right)$ is a monad endomorphism of $\left(T_{r}, m_{r}, u_{r}\right)$.

Note that in the representation of $\Phi$, the string corresponding to $T_{r}$ crosses over the other one. If $\Phi$ is an isomorphism, then the string representation of $\Phi^{-1}$ is obtained from that of $\Phi$ by an up-down reflection.

As it was proven in [1], a distributive law $\Phi: T_{r} T_{l} \rightarrow T_{l} T_{r}$ as in Definition 1.3 induces a monad structure on the composite functor $T_{l} T_{r}$, with the multiplication $m$ and unit $u$, whose string representations are as follows.

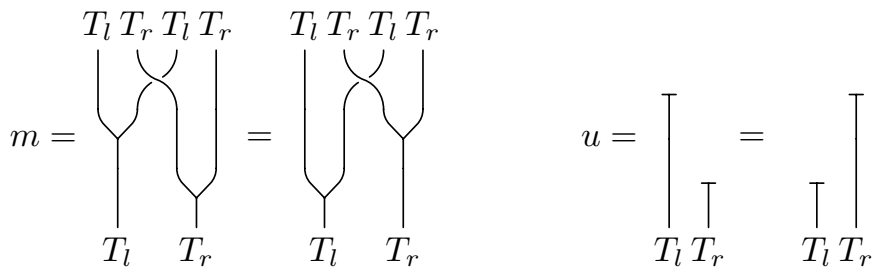

The following well-known lemma describes morphisms between such composite monads.

Lemma 1.4. Let $\left(T_{l}, m_{l}, u_{l}\right),\left(T_{r}, m_{r}, u_{r}\right)$ be two monads on the same category $\mathcal{M}$ and let $\left(T_{l}^{\prime}, m_{l}^{\prime}, u_{l}^{\prime}\right),\left(T_{r}^{\prime}, m_{r}^{\prime}, u_{r}^{\prime}\right)$ be two monads on $\mathcal{M}^{\prime}$. Let $\Phi: T_{r} T_{l} \rightarrow T_{l} T_{r}$ and $\Phi^{\prime}: T_{r}^{\prime} T_{l}^{\prime} \rightarrow T_{l}^{\prime} T_{r}^{\prime}$ be distributive laws. The following assertions are equivalent for monad morphisms $\left(G, q_{l}\right):\left(T_{l}^{\prime}, m_{l}^{\prime}, u_{l}^{\prime}\right) \rightarrow\left(T_{l}, m_{l}, u_{l}\right)$ and $\left(G, q_{r}\right):\left(T_{r}^{\prime}, m_{r}^{\prime}, u_{r}^{\prime}\right) \rightarrow$ $\left(T_{r}, m_{r}, u_{r}\right)$ :

(i) $\left(G, q_{l} T_{r}^{\prime} \circ T_{l} q_{r}\right)$ is a monad morphism $T_{l}^{\prime} T_{r}^{\prime} \rightarrow T_{l} T_{r}, c f$. (1.1). 
(ii) The following identity holds true.

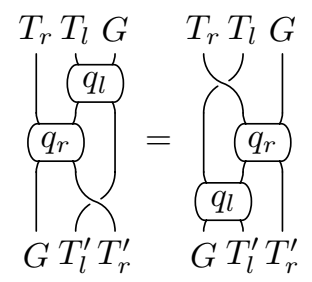

Distributive laws $\left(T_{l}, T_{r}, \Phi\right)$ as objects, with triples $\left(G, q_{l}, q_{r}\right)$ satisfying the equivalent conditions in Lemma 1.4 as morphisms between them, constitute a category which can be regarded again as a full subcategory in the category of 2-functors and lax natural transformations. To this end, consider now a 2-category $\mathscr{L}$ of a single 0 -cell $o$, generated by two monads $\left(t_{l}, \mu_{l}, \eta_{l}\right)$ and $\left(t_{r}, \mu_{r}, \eta_{r}\right)$ on $o$ and a 2-cell $\phi: t_{r} t_{l} \rightarrow t_{l} t_{r}$ such that

$$
\phi \circ \mu_{r} t_{l}=t_{l} \mu_{r} \circ \phi t_{r} \circ t_{r} \phi, \quad \phi \circ \eta_{r} t_{l}=t_{l} \eta_{r}
$$

and

$$
\phi \circ t_{r} \mu_{l}=\mu_{l} t_{r} \circ t_{l} \phi \circ \phi t_{l}, \quad \phi \circ t_{r} \eta_{l}=\eta_{l} t_{r}
$$

(for a diagrammatic representation see Definition 1.3), i.e., such that $\phi$ is a distributive law. A 2-functor $K: \mathscr{L} \rightarrow$ Cat is the same as a pair of monads on the category $K o$ related by a distributive law $K \phi$. A lax natural transformation between such 2-functors is the same as a pair of monad morphisms as in Lemma 1.4.

The following definition is quoted from [7].

Definition 1.5. Consider two monads $\left(T_{l}, m_{l}, u_{l}\right)$ and $\left(T_{r}, m_{r}, u_{r}\right)$ on the same category $\mathcal{M}$ and a distributive law $\Phi: T_{r} T_{l} \rightarrow T_{l} T_{r}$. A $\Phi$-algebra is a pair consisting of an object $X$ of $\mathcal{M}$ and a morphism $\xi: T_{r} X \rightarrow T_{l} X$ such that the following diagrams commute:
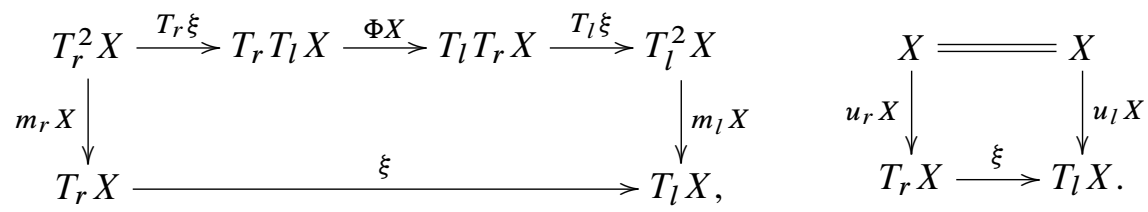

If $T_{l}$ is equal to the identity functor $\mathcal{M}$ then there is a trivial distributive law $\Phi=T_{r}$. In this case $\Phi$-algebras are the same as $T_{r}$-algebras.

A distributive law $\Phi: T_{r} T_{l} \rightarrow T_{l} T_{r}$, between monads on $\mathcal{M}$, induces a distributive law $\operatorname{Cat}(\Phi,-)$ : $\operatorname{Cat}\left(T_{l},-\right) \operatorname{Cat}\left(T_{r},-\right) \rightarrow \operatorname{Cat}\left(T_{r},-\right) \operatorname{Cat}\left(T_{l},-\right)$ between monads on $\operatorname{Cat}(\mathcal{M},-)$ and a distributive law $\operatorname{Cat}(-, \Phi): \operatorname{Cat}\left(-, T_{r}\right) \operatorname{Cat}\left(-, T_{l}\right) \rightarrow$ Cat $\left(-, T_{l}\right)$ Cat $\left(-, T_{r}\right)$ between monads on $\operatorname{Cat}(-, \mathcal{M})$. Algebras for these induced distributive laws are called right and left $\Phi$-module functors, respectively. Explicitly, 
a right $\Phi$-module functor is a pair consisting of a functor $\sqcap: \mathcal{M} \rightarrow \mathcal{C}$ (where $\mathcal{C}$ is any category) and a natural transformation $i: \sqcap T_{l} \rightarrow \sqcap T_{r}$ such that the following relations hold true.
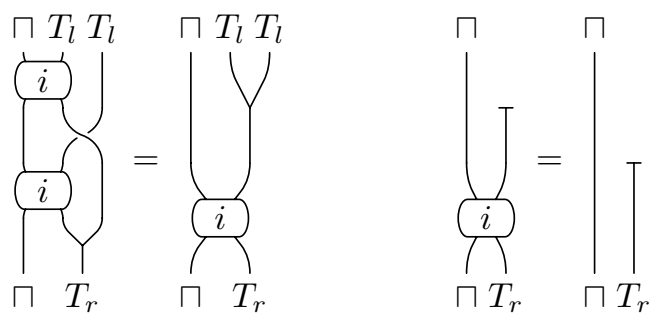

A left $\Phi$-module functor is a pair, consisting of a functor $\sqcup: \mathscr{D} \rightarrow \mathcal{M}$ (where $\mathscr{D}$ is any category) and a natural transformation $w: T_{r} \sqcup \rightarrow T_{l} \sqcup$ such that the following relations hold true.
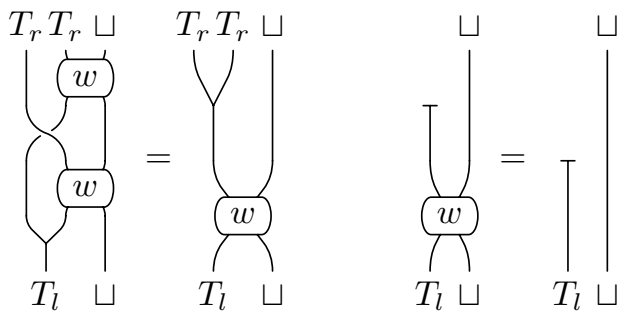

Once again, right or left $\Phi$-module functors can be described as 2-functors from an appropriately chosen 2-category to Cat. Consider a 2-category $\mathcal{R}$, with two 0 cells $o$ and $c$, generated by two monads $\left(t_{l}, \mu_{l}, \eta_{l}\right)$ and $\left(t_{r}, \mu_{r}, \eta_{r}\right)$ on $o$ related by a distributive law $\phi: t_{r} t_{l} \rightarrow t_{l} t_{r}$, and a further 1-cell $p: o \rightarrow c$ together with a 2-cell $\iota: p t_{l} \rightarrow p t_{r}$ such that

$$
\iota \circ p \mu_{l}=p \mu_{r} \circ \iota t_{r} \circ p \phi \circ \iota t_{l} \quad \text { and } \iota \circ p \eta_{l}=p \eta_{r}
$$

(for a diagrammatic representation see (1.3)), i.e., such that $(p, \iota)$ is an $\mathcal{R}(\phi, c)$ algebra in $\mathcal{R}(o, c)$. A 2 -functor $K$ from $\mathcal{R}$ to Cat can be described then as a pair of monads on the category $K o$, related by a distributive law $K \phi$, together with a right $K \phi$-module functor $(K p, K \iota)$. A 2 -functor from the horizontal opposite of $\mathcal{R}$ to Cat corresponds to a pair of monads related by a distributive law and a left module functor for it.

Making use of the above observations, we can define morphisms between right or left $\Phi$-module functors as lax natural transformations between the corresponding 2 -functors. Explicitly, this yields the following.

Definition 1.6. Consider two monads $\left(T_{l}, m_{l}, u_{l}\right)$ and $\left(T_{r}, m_{r}, u_{r}\right)$ on the same category $\mathcal{M}$ and two monads $\left(T_{l}^{\prime}, m_{l}^{\prime}, u_{l}^{\prime}\right)$ and $\left(T_{r}^{\prime}, m_{r}^{\prime}, u_{r}^{\prime}\right)$ on $\mathcal{M}^{\prime}$. Let $\Phi: T_{r} T_{l} \rightarrow T_{l} T_{r}$ and $\Phi^{\prime}: T_{r}^{\prime} T_{l}^{\prime} \rightarrow T_{l}^{\prime} T_{r}^{\prime}$ be distributive laws. 
A morphism from a right $\Phi^{\prime}$-module functor $\left(\Pi^{\prime}: \mathcal{M}^{\prime} \rightarrow \mathcal{C}^{\prime}, i^{\prime}: \Pi^{\prime} T_{l}^{\prime} \rightarrow\right.$ $\left.\Pi^{\prime} T_{r}^{\prime}\right)$ to a right $\Phi$-module functor $\left(\sqcap: \mathcal{M} \rightarrow \mathcal{\mathcal { M }}, i: \sqcap T_{l} \rightarrow \sqcap T_{r}\right)$ is a quintuple $\left(G, q_{l}, q_{r}, \wedge, \pi\right)$, where $\left(G, q_{l}\right):\left(T_{l}^{\prime}, m_{l}^{\prime}, u_{l}^{\prime}\right) \rightarrow\left(T_{l}, m_{l}, u_{l}\right)$ and $\left(G, q_{r}\right)$ : $\left(T_{r}^{\prime}, m_{r}^{\prime}, u_{r}^{\prime}\right) \rightarrow\left(T_{r}, m_{r}, u_{r}\right)$ are monad morphisms, $\wedge: \ell^{\prime} \rightarrow \ell$ is a functor and $\pi: \sqcap G \rightarrow \wedge \sqcap^{\prime}$ is a natural transformation such that (1.2) and the following relation hold.

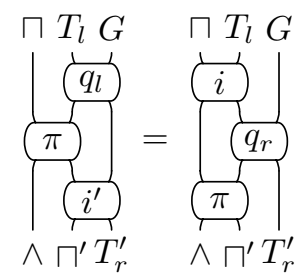

Similarly, a morphism from a left $\Phi^{\prime}$-module functor $\left(\sqcup^{\prime}: \mathscr{D}^{\prime} \rightarrow \mathcal{M}^{\prime}, w^{\prime}: T_{r}^{\prime} \sqcup^{\prime} \rightarrow\right.$ $\left.T_{l}^{\prime} \sqcup^{\prime}\right)$ to a left $\Phi$-module functor $\left(\sqcup: \mathcal{D} \rightarrow \mathcal{M}, w: T_{r} \sqcup \rightarrow T_{l} \sqcup\right)$ is a quintuple $\left(G, q_{l}, q_{r}, \vee, \omega\right)$, where $\left(G, q_{l}\right):\left(T_{l}^{\prime}, m_{l}^{\prime}, u_{l}^{\prime}\right) \rightarrow\left(T_{l}, m_{l}, u_{l}\right)$ and $\left(G, q_{r}\right)$ : $\left(T_{r}^{\prime}, m_{r}^{\prime}, u_{r}^{\prime}\right) \rightarrow\left(T_{r}, m_{r}, u_{r}\right)$ are monad morphisms, $\vee: \mathscr{D}^{\prime} \rightarrow \mathscr{D}$ is a functor and $\omega: \sqcup \vee \rightarrow G \sqcup^{\prime}$ is a natural transformation such that (1.2) and the following relation hold.

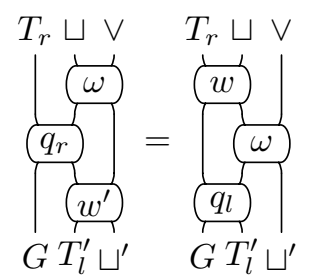

It is immediately clear by their definition as lax natural transformations that morphisms of (left or right) $\Phi$-module functors can be composed in the appropriate sense.

\section{Para-cocyclic objects and $\Phi$-module functors}

In this section we construct a category $\mathcal{A}$, which comes equipped with a functor to a category of para-cocyclic objects in the category of functors. This implies that any object of $\mathcal{A}$ induces a functor from a category $\mathscr{D}$ (of coefficients) to the category of para-cocyclic objects in some category $C$.

Motivated by the constructions in Section 1, consider a 2-category $S$ with three 0 -cells $o, c$ and $d$, generated by two monads on $o$ related by a distributive law $\phi$ and an $S(\phi, c)$-algebra in $S(o, c)$ and an $S(d, \phi)$-algebra in $S(d, o)$. Explicitly, $S$ has 
1-cells depicted in

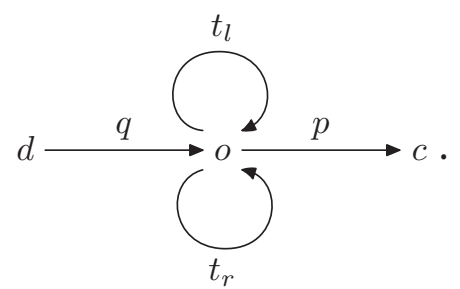

2-cells are horizontal and vertical composites of identity 2-cells and the following 2-cells:

$$
\begin{gathered}
\mu_{l}: t_{l}^{2} \Rightarrow t_{l}, \quad \eta_{l}: o \Rightarrow t_{l}, \quad \mu_{r}: t_{r}^{2} \Rightarrow t_{r}, \quad \eta_{r}: o \Rightarrow t_{r} \\
\phi: t_{r} t_{l} \Rightarrow t_{l} t_{r}, \quad \iota: p t_{l} \Rightarrow p t_{r}, \quad \varpi: t_{r} q \Rightarrow t_{l} q .
\end{gathered}
$$

On these generating 2-cells one imposes three types of relations. The first one
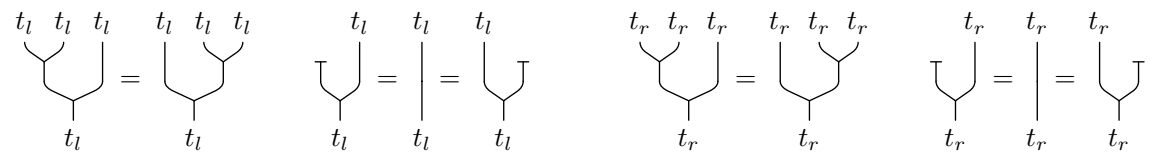

means that $\left(t_{l}, \mu_{l}, \eta_{l}\right)$ and $\left(t_{r}, \mu_{t}, \eta_{t}\right)$ are monads in $S$. One also imposes the relations
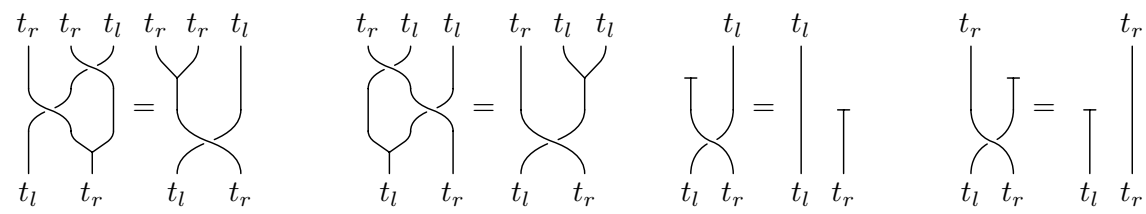

so that $\phi$ is a distributive law. Finally, the relations
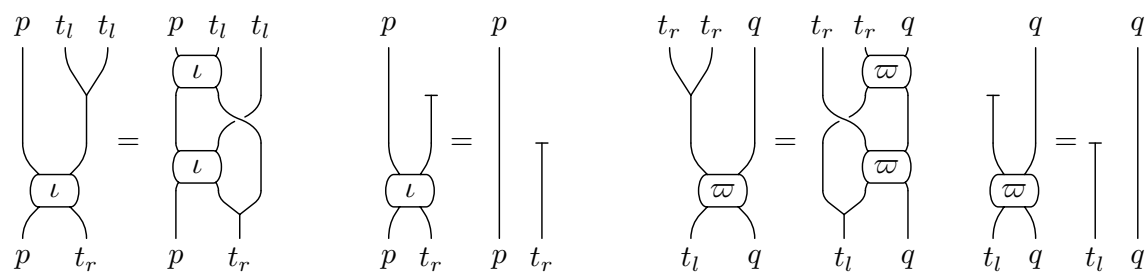

mean that $(p, \iota)$ is $\delta(\phi, c)$-algebra in $\delta(o, c)$ and $(q, \varpi)$ is an $\delta(d, \phi)$-algebra in $S(d, o)$.

We define a category $\mathcal{A}$ as the opposite of the category of 2-functors from $S$ to Cat and lax natural transformations between them. Explicitly, this means the following.

Definition 2.1. The category $\mathcal{A}$ is defined to have objects $\left(T_{l}, T_{r}, \Phi, \sqcap, i, \sqcup, w\right)$, where 
- $T_{l}$ and $T_{r}$ are monads on the same category $\mathcal{M}$;

- $\Phi: T_{r} T_{l} \rightarrow T_{l} T_{r}$ is a distributive law;

- $\left(\sqcap: \mathcal{M} \rightarrow \mathcal{C}, i: \sqcap T_{l} \rightarrow \sqcap T_{r}\right)$ is a right $\Phi$-module functor;

- $\left(\sqcup: \mathscr{D} \rightarrow \mathcal{M}, w: T_{r} \sqcup \rightarrow T_{l} \sqcup\right)$ is a left $\Phi$-module functor.

By a morphism $\left(T_{l}, T_{r}, \Phi, \sqcap, i, \sqcup, w\right) \rightarrow\left(T_{l}^{\prime}, T_{r}^{\prime}, \Phi^{\prime}, \sqcap^{\prime}, i^{\prime}, \sqcup^{\prime}, w^{\prime}\right)$ we mean a datum $\left(G, q_{l}, q_{r}, \wedge, \pi, \vee, \omega\right)$ such that

- $\left(G, q_{l}, q_{r}, \wedge, \pi\right)$ is a morphism from the right $\Phi^{\prime}$-module functor $\left(\Pi^{\prime}, i^{\prime}\right)$ to the right $\Phi$-module functor $(\sqcap, i)$ (cf. Definition 1.6);

- $\left(G, q_{l}, q_{r}, \vee, \omega\right)$ is a morphism from the left $\Phi^{\prime}$-module functor $\left(\sqcup^{\prime}, w^{\prime}\right)$ to the left $\Phi$-module functor $(\sqcup, w)$ (cf. Definition 1.6).

Recall that a para-cocyclic object in a category $\varphi$ consists of a family $\left\{Z^{n}\right\}_{n}$ of objects in $\mathcal{C}$, for all non-negative integers $n$, and morphisms

$$
d^{k}: Z^{n-1} \rightarrow Z^{n}, \quad s^{k}: Z^{n+1} \rightarrow Z^{n} \text { for } k=0, \ldots, n,
$$

called coface and codegeneracy morphisms, respectively, satisfying cosimplicial relations, together with so called para-cocyclic morphisms $t^{n}: Z^{n} \rightarrow Z^{n}$ for all $n \geq 0$, which satisfy

$t^{n} \circ d^{0}=d^{n}, t^{n} \circ d^{k}=d^{k-1} \circ t^{n-1}, t^{n} \circ s^{0}=s^{n} \circ t^{n+1} \circ t^{n+1}, t^{n} \circ s^{k}=s^{k-1} \circ t^{n+1}$

for all $k=1, \ldots, n$. A morphism $\left(Z^{*}, d^{*}, s^{*}, t^{*}\right) \rightarrow\left(Z^{\prime *}, d^{\prime *}, s^{\prime *}, t^{\prime *}\right)$ is a family of morphisms $\left\{f^{n}: Z^{n} \rightarrow Z^{\prime n}\right\}_{n \geq 0}$ in $\mathcal{C}$, compatible with the coface, codegeneracy and para-cocyclic morphisms in the evident sense.

Definition 2.2. The category $\overline{\mathcal{P}}$ is defined to have objects that are para-cocyclic objects in the category of functors. That is, for any non-negative integer $n$, a functor $Z^{n}: \mathcal{D} \rightarrow \mathcal{C}$ together with natural transformations $d^{k}: Z^{n-1} \rightarrow Z^{n}, s^{k}: Z^{n+1} \rightarrow$ $Z^{n}, t^{n}: Z^{n} \rightarrow Z^{n}$ for $0 \leq k \leq n$, satisfying the defining relations of a para-cocyclic object.

Morphisms from $\left(Z^{*}: \mathcal{D} \rightarrow \mathcal{C}, d^{*}, s^{*}, t^{*}\right)$ to $\left(Z^{* *}: \mathscr{D}^{\prime} \rightarrow \mathcal{C}^{\prime}, d^{\prime *}, s^{\prime *}, t^{\prime *}\right)$ are triples $\left(\wedge, \vee, \xi^{*}\right)$, where $\wedge: \mathcal{C}^{\prime} \rightarrow \mathcal{C}$ and $\vee: \mathscr{D}^{\prime} \rightarrow \mathscr{D}$ are functors and $\xi^{*}$ : $\left(Z^{*} \vee, d^{*} \vee, s^{*} \vee, t^{*} \vee\right) \rightarrow\left(\wedge Z^{\prime *}, \wedge d^{\prime *}, \wedge s^{\prime *}, \wedge t^{\prime *}\right)$ is a morphism of para-cocyclic objects.

In terms of $\Phi: T_{r} T_{l} \rightarrow T_{l} T_{r}$ and $q_{l}: T_{l} G \rightarrow G T_{l}^{\prime}$, we define inductively some new natural transformations: Let $q_{l}^{0}$ be the identity natural transformation $G \rightarrow G$ and $\Phi^{0}$ be the identity natural transformation $T_{r} \rightarrow T_{r}$. Put $q_{l}^{1}:=q_{l}$ and $\Phi^{1}:=\Phi$. For every $n>1$ we now define $\Phi^{n}: T_{r} T_{l}^{n} \rightarrow T_{l}^{n} T_{r}$ and $q_{l}^{n}: T_{l}^{n} G \rightarrow G T_{l}^{\prime n}$ by $\Phi^{n}:=T_{l}^{n-1} \Phi \circ \Phi^{n-1} T_{l}$ and $q_{l}^{n}:=q_{l} T_{l}^{\prime n-1} \circ T_{l} q_{l}^{n-1}$, respectively. For these 
natural transformations we will use the string diagrams in the figure below.

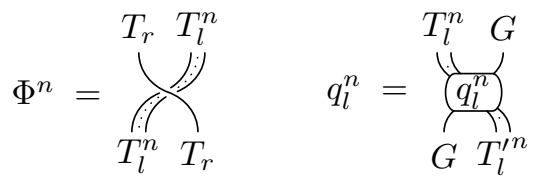

Note that in this figure each stripe represents a bunch of $n$ strings, each one representing the functor $T_{l}$.

Theorem 2.3. There is a functor $\mathbb{Z}^{*}: \mathcal{A} \rightarrow \overline{\mathcal{P}}$ with object map

$$
\left(T_{l}, T_{r}, \Phi, \sqcap: \mathcal{M} \rightarrow \mathcal{C}, i, \sqcup: \mathcal{D} \rightarrow \mathcal{M}, w\right) \mapsto\left(\sqcap T_{l}^{*+1} \sqcup: \mathscr{D} \rightarrow \mathcal{C}, d^{*}, s^{*}, t^{*}\right)
$$

The functor $\mathbb{Z}^{*}$ takes a morphism

$$
\left(G, q_{l}, q_{r}, \wedge, \pi, \vee, \omega\right):\left(T_{l}, T_{r}, \Phi, \sqcap, i, \sqcup, w\right) \rightarrow\left(T_{l}^{\prime}, T_{r}^{\prime}, \Phi^{\prime}, \sqcap^{\prime}, i^{\prime}, \sqcup^{\prime}, w^{\prime}\right)
$$

to the triple $\left(\wedge, \vee, \xi^{*}\right)$. At every degree $n \geq 0$ and for $0 \leq k \leq n$, the coface morphisms $d^{k}$, the codegeneracy morphisms $\bar{s}^{k}$, the para-cocyclic morphism $t^{n}$ and the morphism $\xi^{n}$ are given by the natural transformations below.
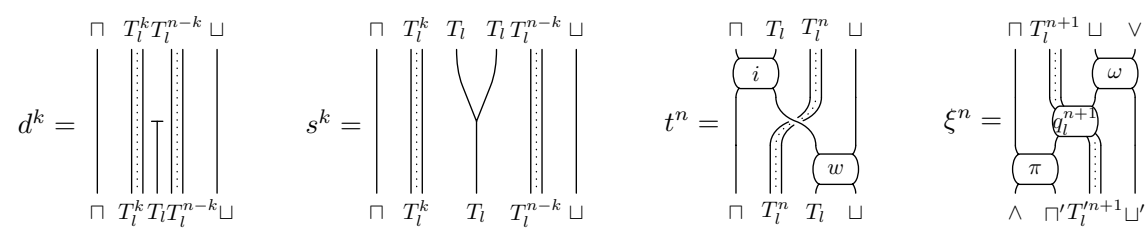

Proof. The datum $\left(\sqcap T_{l}^{*+1} \sqcup, d^{*}, s^{*}\right)$ is obviously a cosimplex in the category of functors, cf. [25]. Its para-cocyclicity is checked with the same steps in [4], Theorem 1.10. It remains to show that $\xi^{*}$ is a morphism of para-cocyclic objects. Its compatibility with the coface and codegeneracy morphisms follows by naturality and monad morphism property of $q_{l}$, see the following diagrammatic computations.
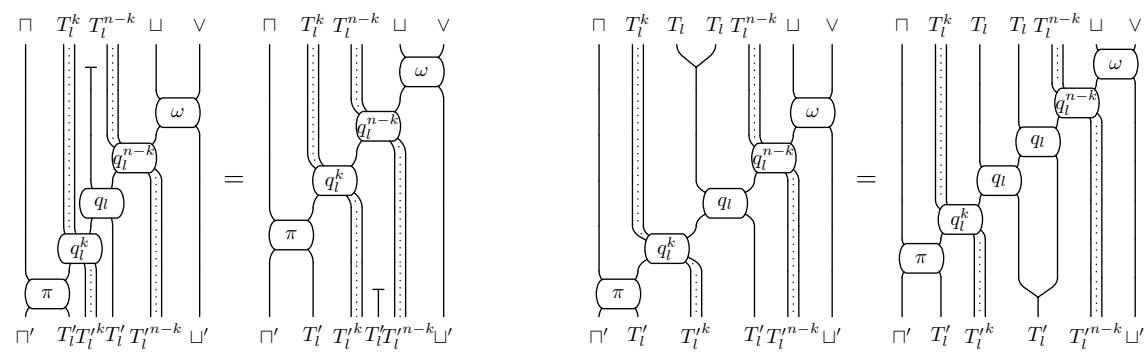
Compatibility with the para-cocyclic morphisms is proved in the figure below.
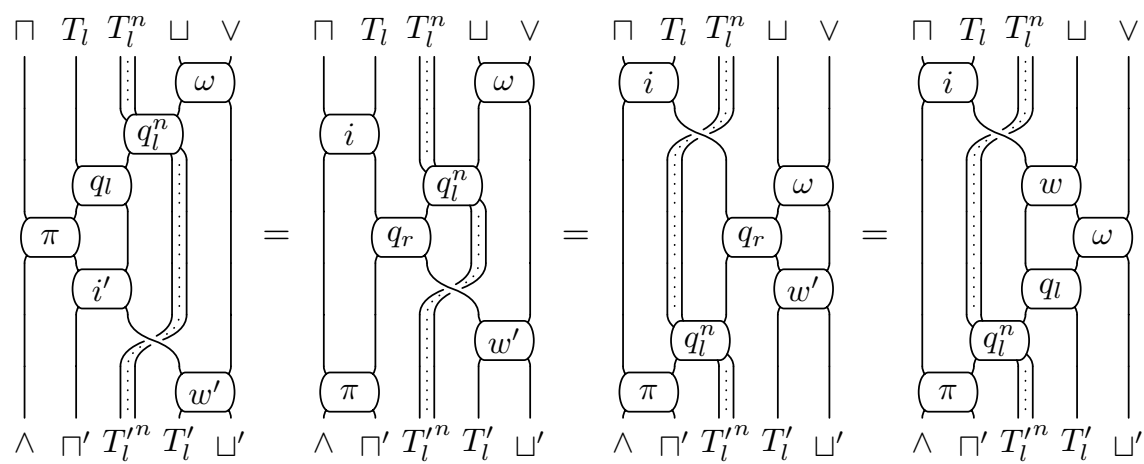

It follows by using naturality and (1.5) in the first equality, then applying (1.2) repeatedly in the second equality, and using in the last equality (1.6) together with naturality.

Corollary 2.4. Any object $\left(T_{l}, T_{r}, \Phi, \sqcap: \mathcal{M} \rightarrow \mathcal{C}, i, \sqcup: \mathcal{D} \rightarrow \mathcal{M}, w\right)$ of the category $\mathcal{A}$ determines a functor from $\mathcal{D}$ to the category of para-cocyclic objects in $C$. The objects of $\mathfrak{D}$ play the role of coefficients for the resulting para-cocyclic object in $\mathcal{C}$.

\section{Examples from Hopf cyclic theory}

In this section we list some examples of objects in the category $\mathcal{A}$ in Definition 2.1, arising from Hopf cyclic theory (of bialgebroids, hence in particular of bialgebras). They give rise to families of para-cocyclic objects in the category Mod- $k$ of modules over a commutative ring $k$. They extend examples in [13], [12], [16] and [5].

Let $R$ be an algebra over a commutative ring $k$. The tensor product of two $k$ modules will be denoted by $\otimes$. Regarding the regular $R$-bimodule $R$ as a right $R^{\mathrm{e}}:=R \otimes R^{\mathrm{op}}$-module, and regarding any $R$-bimodule as a left $R^{\mathrm{e}}$-module, we can define a functor $R \otimes_{R^{\mathrm{e}}}(-): R$-Mod- $R \rightarrow$ Mod- $k$. Applying it to the $R$-module tensor product of two $R$-bimodules $M$ and $N$, it yields the so called cyclic $R$-module tensor product. Throughout the paper, it will be denoted by

$$
M \hat{\otimes}_{R} N:=R \otimes_{R^{\mathrm{e}}}\left(M \otimes_{R} N\right) \cong M \otimes_{R^{\mathrm{e}}} N .
$$

For finitely many $R$-bimodules $\left\{M_{i}\right\}_{i=1, \ldots, n}$, we put

$$
M_{1} \hat{\otimes}_{R} M_{2} \hat{\otimes}_{R} \cdots \hat{\otimes}_{R} M_{n}:=\left(M_{1} \otimes_{R} \cdots \otimes_{R} M_{i-1}\right) \otimes_{R^{\mathrm{e}}}\left(M_{i} \otimes_{R} \cdots \otimes_{R} M_{n}\right),
$$

where the right-hand side yields the same $k$-module for any $i=1, \ldots, n$ (defining the 0 -fold tensor product to be equal to $R$ ).

For a short review of modules, comodules and contramodules of a bialgebroid, we refer to the Appendix. Throughout, actions in modules are denoted by juxtaposition 
and for coactions in comodules we use a Sweedler type index notation, with implicit summation understood.

The first example of an object in the category $\mathcal{A}$ in Definition 2.1 arises from Theorem 2.4 of [4].

Example 3.1. Let $B$ be a left bialgebroid over a $k$-algebra $L$ and $A$ be a left $B$-module algebra. Then $A$ is in particular an $L$-ring, with multiplication $\mu: A \otimes_{L} A \rightarrow A$ and unit $\eta: L \rightarrow A$. An object in $\mathcal{A}$ is given by the following data:

- the monads $T_{l}=A \otimes_{L}(-)$ and $T_{r}=(-) \otimes_{L} A$ on $L$-Mod- $L$, with monad structures

$$
\mu \otimes_{L}(-): T_{l}^{2} \rightarrow T_{l}, \quad \eta \otimes_{L}(-): L-\operatorname{Mod}-L \rightarrow T_{l}
$$

and

$$
(-) \otimes_{L} \mu: T_{r}^{2} \rightarrow T_{r}, \quad(-) \otimes_{L} \eta: L-\operatorname{Mod}-L \rightarrow T_{r},
$$

respectively;

- the trivial distributive law $\Phi=A \otimes_{L}(-) \otimes_{L} A: T_{r} T_{l} \rightarrow T_{l} T_{r}$;

- the right $\Phi$-module functor $(\sqcap, i)$, where $\sqcap=L \otimes_{L^{e}}(-): L$-Mod- $L \rightarrow \operatorname{Mod}-k$ and

$$
i_{P}: A \hat{\otimes}_{L} P \rightarrow P \hat{\otimes}_{L} A, \quad a \hat{\otimes}_{L} p \mapsto p \hat{\otimes}_{L} a,
$$

for any $L$-bimodule $P$;

- the left $\Phi$-module functor $(\sqcup, w)$, where $\sqcup: B$-Comod $\rightarrow L$-Mod- $L$ is the forgetful functor and

$$
w_{M}: M \otimes_{L} A \rightarrow A \otimes_{L} M, \quad m \otimes_{L} a \mapsto m_{[-1]} a \otimes_{L} m_{[0]}
$$

for any left $B$-comodule $M$ with coaction $m \mapsto m_{[-1]} \otimes_{L} m_{[0]}$.

Applying the functor $Z^{*}$ in Theorem 2.3, we obtain a para-cocyclic object in Mod- $k$ for any left $B$-comodule $M$. At degree $n$, it is the $k$-module $A^{\otimes_{L} n+1} \widehat{\otimes}_{L} M$. Coface and codegeneracy maps are

$$
\begin{aligned}
& d^{k}\left(a_{0} \hat{\otimes}_{L} a_{1} \hat{\otimes}_{L} \cdots \hat{\otimes}_{L} a_{n-1} \hat{\otimes}_{L} m\right) \\
& \quad=a_{0} \hat{\otimes}_{L} \cdots \hat{\otimes}_{L} a_{k-1} \hat{\otimes}_{L} 1_{A} \hat{\otimes}_{L} a_{k} \hat{\otimes}_{L} \cdots \hat{\otimes}_{L} a_{n-1} \hat{\otimes}_{L} m, \\
& s^{k}\left(a_{0} \hat{\otimes}_{L} a_{1} \hat{\otimes}_{L} \cdots \hat{\otimes}_{L} a_{n+1} \hat{\otimes}_{L} m\right) \\
& \quad=a_{0} \hat{\otimes}_{L} \cdots \hat{\otimes}_{L} a_{k-1} \hat{\otimes}_{L} a_{k} a_{k+1} \hat{\otimes}_{L} a_{k+2} \hat{\otimes}_{L} \cdots \hat{\otimes}_{L} a_{n+1} \hat{\otimes}_{L} m
\end{aligned}
$$

for $k=0, \ldots, n$. The para-cocyclic operator comes out as

$$
t^{n}\left(a_{0} \hat{\otimes}_{L} a_{1} \hat{\otimes}_{L} \cdots \hat{\otimes}_{L} a_{n} \hat{\otimes}_{L} m\right)=a_{1} \hat{\otimes}_{L} \cdots \hat{\otimes}_{L} a_{n} \hat{\otimes}_{L} m_{[-1]} a_{0} \hat{\otimes}_{L} m_{[0]} .
$$

The next example is obtained from [4], Theorem 2.7. 
Example 3.2. Let $B$ be a right bialgebroid over a $k$-algebra $R$ and $(A, \mu, \eta)$ be a right $B$-comodule algebra (hence in particular an $R$-ring). For the $B$-coaction on $A$, use the notation $a \mapsto a^{[0]} \otimes_{R} a^{[1]}$. An object in $\mathcal{A}$ is given by the following data:

- the same monads $T_{l}=A \otimes_{R}(-)$ and $T_{r}=(-) \otimes_{R} A$ on $R$-Mod- $R$, introduced in Example 3.1 (replacing $L$ by $R$ );

- the trivial distributive law $\Phi=A \otimes_{R}(-) \otimes_{R} A: T_{r} T_{l} \rightarrow T_{l} T_{r}$;

- the same right $\Phi$-module functor $(\sqcap, i)$, introduced in Example 3.1 (replacing $L$ by $R$ );

- the left $\Phi$-module functor $(\sqcup, w)$, where $\sqcup: \operatorname{Mod}-B \rightarrow R$-Mod- $R$ is the forgetful functor and

$$
w_{N}: N \otimes_{R} A \rightarrow A \otimes_{R} N, \quad m \otimes_{R} a \mapsto a^{[0]} \otimes_{R} m a^{[1]},
$$

for any right $B$-module $N$.

For any right $B$-module $N$, the corresponding para-cocyclic object in Mod- $k$ is, at degree $n, A^{\otimes_{R} n+1} \hat{\otimes}_{R} N$. Coface and codegeneracy maps are given by the same formulae in Example 3.1 (replacing $L$ by $R$ and $M$ by $N$ ). The para-cocyclic operator has the form

$$
t^{n}\left(a_{0} \hat{\otimes}_{R} a_{1} \hat{\otimes}_{R} \cdots \hat{\otimes}_{R} a_{n} \hat{\otimes}_{R} m\right)=a_{1} \hat{\otimes}_{R} \cdots \hat{\otimes}_{R} a_{n} \hat{\otimes}_{R} a_{0}^{[0]} \hat{\otimes}_{R} m a_{0}^{[1]} .
$$

In the following example, for a left $R$-module $P$, a right $R$-module $Q$ and $R$ bimodules $C$ and $D$, the canonical isomorphisms

$$
\operatorname{Hom}_{-, R}\left(C, \operatorname{Hom}_{-, R}(D, Q)\right) \cong \operatorname{Hom}_{-, R}\left(C \otimes_{R} D, Q\right)
$$

and

$$
\operatorname{Hom}_{R,-}\left(C, \operatorname{Hom}_{R,-}(D, P)\right) \cong \operatorname{Hom}_{R,-}\left(D \otimes_{R} C, P\right)
$$

are suppressed.

Example 3.3. Let $B$ be a right bialgebroid over a $k$-algebra $R$ and $(C, \Delta, \epsilon)$ be a right $B$-module coring (hence in particular an $R$-coring). An object in $\mathcal{A}$ is given by the following data:

- the monads $T_{l}:=\operatorname{Hom}_{-, R}(C,-)$ and $T_{r}:=\operatorname{Hom}_{R,-}(C,-)$ on $R$-Mod- $R$, with monad structures

$$
\operatorname{Hom}_{-, R}(\Delta,-): T_{l}^{2} \rightarrow T_{l}, \quad \operatorname{Hom}_{-, R}(\epsilon,-): R-\operatorname{Mod}-R \rightarrow T_{l},
$$

and

$$
\operatorname{Hom}_{R,-}(\Delta,-): T_{r}^{2} \rightarrow T_{r}, \quad \operatorname{Hom}_{R,-}(\epsilon,-): R-\operatorname{Mod}-R \rightarrow T_{r},
$$

respectively; 
- the distributive law

$$
\begin{aligned}
\Phi: \operatorname{Hom}_{R,-}\left(C, \operatorname{Hom}_{-, R}(C,-)\right) & \cong \operatorname{Hom}_{R, R}\left(C \otimes_{k} C,-\right) \\
& \cong \operatorname{Hom}_{-, R}\left(C, \operatorname{Hom}_{R,-}(C,-)\right)
\end{aligned}
$$

given by switching the arguments;

- the right $\Phi$-module functor $(\sqcap, i)$, where $\sqcap=\operatorname{Hom}_{R, R}(R,-): R$-Mod- $R \rightarrow$ Mod- $k$ and

$$
\begin{aligned}
i_{P}: \operatorname{Hom}_{R, R}\left(R, \operatorname{Hom}_{-, R}(C, P)\right) & \cong \operatorname{Hom}_{R, R}(C, P) \\
& \cong \operatorname{Hom}_{R, R}\left(R, \operatorname{Hom}_{R,-}(C, P)\right)
\end{aligned}
$$

is the hom-tensor adjunction natural isomorphism for any $R$-bimodule $P$;

- the left $\Phi$-module functor $(\sqcup, w)$, where $\sqcup: B$-Ctrmod $\rightarrow R$-Mod- $R$ is the forgetful functor and

$$
w_{Q}: \operatorname{Hom}_{R,-}(C, Q) \rightarrow \operatorname{Hom}_{-, R}(C, Q), \quad f \mapsto(c \mapsto \alpha(f(c-))),
$$

for a left $B$-contramodule $(Q, \alpha)$.

For any left $B$-contramodule $(Q, \alpha)$, this yields a para-cocyclic object in Mod- $k$. It is given by $\operatorname{Hom}_{R, R}\left(C^{\otimes_{R} n+1}, Q\right)$, at degree $n$. Coface and codegeneracy maps are

$$
\begin{aligned}
& \left(d^{k} \varphi^{(n-1)}\right)\left(c_{0} \otimes_{R} c_{1} \otimes_{R} \cdots \otimes_{R} c_{n}\right) \\
& \quad=\varphi^{(n-1)}\left(c_{0} \otimes_{R} \cdots \otimes_{R} c_{k-1} \epsilon\left(c_{k}\right) \otimes_{R} c_{k+1} \otimes_{R} \cdots \otimes_{R} c_{n}\right) \\
& \left(s^{k} \varphi^{(n+1)}\right)\left(c_{0} \otimes_{R} c_{1} \otimes_{R} \cdots \otimes_{R} c_{n}\right) \\
& \quad=\varphi^{(n+1)}\left(c_{0} \otimes_{R} \cdots \otimes_{R} c_{k-1} \otimes_{R} \Delta\left(c_{k}\right) \otimes_{R} c_{k+1} \otimes_{R} \cdots \otimes_{R} c_{n}\right)
\end{aligned}
$$

for $\varphi^{(j)} \in \operatorname{Hom}_{R, R}\left(C^{\otimes_{R} j+1}, Q\right)$ and $k=0, \ldots, n$. The para-cocyclic map is equal to

$\left(t^{n} \varphi^{(n)}\right)\left(c_{0} \otimes_{R} c_{1} \otimes_{R} \cdots \otimes_{R} c_{n}\right)=\alpha\left(\varphi^{(n)}\left(c_{n}(-) \otimes_{R} c_{0} \otimes_{R} c_{1} \otimes_{R} \cdots \otimes_{R} c_{n-1}\right)\right)$.

Example 3.4. Let $B$ be a left bialgebroid over a $k$-algebra $L$ and $(C, \Delta, \epsilon)$ be a left $B$-comodule coring (hence in particular an $L$-coring), with $B$-coaction $c \mapsto$ $c_{[-1]} \otimes_{L} c_{[0]}$. An object in $\mathcal{A}$ is given by the following data:

- the same monads $T_{l}:=\operatorname{Hom}_{-, L}(C,-)$ and $T_{r}:=\operatorname{Hom}_{L,-}(C,-)$ on $L-M o d-L$, introduced in Example 3.3 (replacing $R$ by $L$ );

- the same distributive law $\Phi$, introduced in Example 3.3 (replacing $R$ by $L$ );

- the same right $\Phi$-module functor $(\sqcap, i)$, introduced in Example 3.3 (replacing $R$ by $L$ ); 
- the left $\Phi$-module functor $(\sqcup, w)$, where $\sqcup:$ Mod- $B \rightarrow L$-Mod- $L$ is the forgetful functor and for any right $B$-module $N$,

$$
w_{N}: \operatorname{Hom}_{L,-}(C, N) \rightarrow \operatorname{Hom}_{-, L}(C, N), \quad f \mapsto\left(c \mapsto f\left(c_{[0]}\right) c_{[-1]}\right) .
$$

The cosimplicial structure of the para-cocyclic object in Mod- $k$, corresponding to a right $B$-module $N$, is the same as in Example 3.3 (replacing $R$ by $L$ and $Q$ by $N$ ). The para-cocyclic map comes out as

$\left(t^{n} \varphi^{(n)}\right)\left(c_{0} \otimes_{L} c_{1} \otimes_{L} \cdots \otimes_{L} c_{n}\right)=\varphi^{(n)}\left(c_{n[0]} \otimes_{L} c_{0} \otimes_{L} c_{1} \otimes_{L} \cdots \otimes_{L} c_{n-1}\right) c_{n[-1]}$.

Specializing the above four examples to bialgebras instead of bialgebroids, in all of them the functors $\Pi$ become identity functors.

For an $R$-coring $C$, a $C$-bicomodule is an $R$-bimodule $M$, together with a right $C$-coaction $\varrho^{M}: M \rightarrow M \otimes_{R} C$ and a left $C$-coaction ${ }^{M} \varrho: M \rightarrow C \otimes_{R} M$ such that $\varrho^{M}$ is a left $R$-module map, ${ }^{M} \varrho$ is a right $R$-module map and $\left({ }^{M} \varrho \otimes_{R} C\right) \circ \varrho^{M}=$ $\left(C \otimes_{R} \varrho^{M}\right) \circ^{M} \varrho$. Morphisms of bicomodules are right $C$-comodule maps as well as left $C$-comodule maps. The category of $C$-bicomodules is denoted by $C$-Comod- $C$.

Example 3.5. Let $B$ be a right bialgebroid over a $k$-algebra $R$ and $(C, \Delta, \epsilon)$ be a left $B$-comodule coring, hence in particular an $L:=R^{\text {op }}$-coring. An object in $\mathcal{A}$ is given by the following data:

- The monads $T_{l}=C \otimes_{L}(-)$ and $T_{r}=(-) \otimes_{L} C$ on $C$-Comod- $C$. For a $C$-bicomodule $\left(M,{ }^{M} \varrho, \varrho^{M}\right), T_{l} M=C \otimes_{L} M$ is a $C$-bicomodule via the left and right coactions

$$
c \otimes_{L} m \mapsto \Delta(c) \otimes_{L} m \quad \text { and } \quad c \otimes_{L} m \mapsto c \otimes_{L} Q^{M}(m) .
$$

The monad structure of $T_{l}$ is given by the multiplication and unit

$$
C \otimes_{L} \epsilon \otimes_{L} M: T_{l}^{2} M \rightarrow T_{l} M \quad \text { and } \quad M_{\varrho}: M \rightarrow T_{l} M .
$$

Symmetrically, $T_{r} M=M \otimes_{L} C$ is a $C$-bicomodule via the left and right coactions

$$
m \otimes_{L} c \mapsto{ }^{M} \varrho(m) \otimes_{L} c \quad \text { and } \quad m \otimes_{L} c \mapsto m \otimes_{L} \Delta(c) .
$$

The monad structure of $T_{r}$ is given by the multiplication and unit

$$
M \otimes_{L} \epsilon \otimes_{L} C: T_{r}^{2} M \rightarrow T_{r} M \quad \text { and } \quad \varrho^{M}: M \rightarrow T_{r} M .
$$

- The trivial distributive law $\Phi=C \otimes_{L}(-) \otimes_{L} C$.

- The right $\Phi$-module functor $\sqcap: C$-Comod- $C \rightarrow$ Mod- $k$ given by the equalizer

$$
\sqcap M \longrightarrow L \hat{\otimes}_{L} M \frac{L \hat{\otimes}_{L} \varrho^{M}}{\stackrel{L \hat{\otimes}_{L} M_{\varrho}}{\underset{ }{\rightleftharpoons}}} C \hat{\otimes}_{L} M \cong M \hat{\otimes}_{L} C
$$


for any $C$-bicomodule $M$. The natural transformation $i$ is given by the isomorphism

$$
i: \sqcap T_{l} \cong L \hat{\otimes}_{L}(-) \cong(-) \hat{\otimes}_{L} L \cong \sqcap T_{r}
$$

- The left $\Phi$-module functor $\sqcup=(-) \otimes_{L} C$ : Mod- $B \rightarrow C$-Comod- $C$. For the left $B$-coaction on $C$, introduce the index notation $c \mapsto c^{[-1]} \otimes_{R} c^{[0]}$ and for the comultiplication in $C$ write $\Delta(c)=c_{\langle 1\rangle} \otimes_{L} c_{\langle 2\rangle}$. For any right $B$-module $N, \sqcup N=N \otimes_{L} C$ is a $C$-bicomodule via the left and right coactions

$m \otimes_{L} c \mapsto c_{\langle 1\rangle}^{[0]} \otimes_{L} m c_{\langle 1\rangle}^{[-1]} \otimes_{L} c_{\langle 2\rangle} \quad$ and $\quad m \otimes_{L} c \mapsto m \otimes_{L} c_{\langle 1\rangle} \otimes_{L} c_{\langle 2\rangle}$.

The natural transformation $w:(-) \otimes_{L} C \otimes_{L} C \rightarrow C \otimes_{L}(-) \otimes_{L} C$ is given by

$$
w_{N}\left(m \otimes_{L} c \otimes_{L} d\right)=c^{[0]} \otimes_{L} m c^{[-1]} \otimes_{L} d .
$$

For any right $B$-module $N$, this yields a para-cocyclic object in Mod- $k$. At degree $n$, it is given by $\sqcap\left(C^{\otimes_{L} n+1} \otimes_{L} N \otimes_{L} C\right) \cong C^{\otimes_{L} n+1} \widehat{\otimes}_{L} N$. For every $0 \leq k \leq n-1$, the corresponding coface map is

$$
\begin{aligned}
& d^{k}\left(c_{0} \hat{\otimes}_{L} \cdots \hat{\otimes}_{L} c_{n-1} \hat{\otimes}_{L} m\right) \\
& \quad=c_{0} \hat{\otimes}_{L} \cdots \hat{\otimes}_{L} c_{k-1} \hat{\otimes}_{L} \Delta\left(c_{k}\right) \hat{\otimes}_{L} c_{k+1} \hat{\otimes}_{L} \cdots \hat{\otimes}_{L} c_{n-1} \hat{\otimes}_{L} m,
\end{aligned}
$$

while

$$
\begin{aligned}
& d^{n}\left(c_{0} \hat{\otimes}_{L} \cdots \hat{\otimes}_{L} c_{n-1} \hat{\otimes}_{L} m\right) \\
& \quad=c_{0\langle 2\rangle} \hat{\otimes}_{L} c_{1} \hat{\otimes}_{L} \cdots \hat{\otimes}_{L} c_{n-1} \hat{\otimes}_{L} c_{0\langle 1\rangle}{ }^{[0]} \hat{\otimes}_{L} m c_{0\langle 1\rangle}{ }^{[-1]}
\end{aligned}
$$

If $0 \leq k \leq n$, then the codegeneracy map $s^{k}$ is given by

$$
\begin{aligned}
& s^{k}\left(c_{0} \hat{\otimes}_{L} \cdots \hat{\otimes}_{L} c_{n+1} \hat{\otimes}_{L} m\right) \\
& \quad=c_{0} \hat{\otimes}_{L} \cdots \hat{\otimes}_{L} c_{k-1} \hat{\otimes}_{L} c_{k} \epsilon\left(c_{k+1}\right) \hat{\otimes}_{L} c_{k+2} \hat{\otimes}_{L} \cdots \hat{\otimes}_{L} c_{n+1} \hat{\otimes}_{L} m .
\end{aligned}
$$

The para-cocyclic map is

$$
t^{n}\left(c_{0} \hat{\otimes}_{L} \cdots \hat{\otimes}_{L} c_{n} \hat{\otimes}_{L} m\right)=c_{1} \hat{\otimes}_{L} \cdots \hat{\otimes}_{L} c_{n} \hat{\otimes}_{L} c_{0}^{[0]} \hat{\otimes}_{L} m c_{0}^{[-1]} .
$$

Example 3.6. Let $B$ be a right bialgebroid over a $k$-algebra $R$ and $(C, \Delta, \epsilon)$ be a right $B$-module coring, hence in particular an $R$-coring. An object in $\mathcal{A}$ is given by the following data:

- The same monads $T_{l}=C \otimes_{R}(-)$ and $T_{r}=(-) \otimes_{R} C$ on $C$-Comod- $C$, introduced in Example 3.5 (replacing $L$ by $R$ ).

- The trivial distributive law $\Phi=C \otimes_{R}(-) \otimes_{R} C$.

- The same right $\Phi$-module functor $(\sqcap, i)$, introduced in Example 3.5 (replacing $L$ by $R$ ). 
- The left $\Phi$-module functor $\sqcup=(-) \otimes_{R} C: B$-Comod $\rightarrow C$-Comod- $C$. For a left $B$-comodule $M$, with coaction $m \mapsto m^{[-1]} \otimes_{R} m^{[0]}$, using the notation $\Delta(c)=c_{\langle 1\rangle} \otimes_{R} c_{\langle 2\rangle}, \sqcup M=M \otimes_{R} C$ is a $C$-bicomodule with left and right coactions

$m \otimes_{R} c \mapsto c_{\langle 1\rangle} m^{[-1]} \otimes_{R} m^{[0]} \otimes_{R} c_{\langle 2\rangle} \quad$ and $\quad m \otimes_{R} c \mapsto m \otimes_{R} c_{\langle 1\rangle} \otimes_{R} c_{\langle 2\rangle}$.

The natural transformation $w:(-) \otimes_{R} C \otimes_{R} C \rightarrow C \otimes_{R}(-) \otimes_{R} C$ is given by

$$
w_{N}\left(m \otimes_{R} c \otimes_{R} d\right)=c m^{[-1]} \otimes_{R} m^{[0]} \otimes_{R} d .
$$

For any left $B$-comodule $M$ this determines a para-cocyclic object in Mod- $k$. At degree $n$, it is given by $\sqcap\left(C^{\otimes_{R} n+1} \otimes_{R} M \otimes_{R} C\right) \cong C^{\otimes_{R} n+1} \widehat{\otimes}_{R} M$. For every $0 \leq k \leq n-1$, the corresponding coface map is

$$
\begin{aligned}
& d^{k}\left(c_{0} \hat{\otimes}_{R} \cdots \hat{\otimes}_{R} c_{n-1} \hat{\otimes}_{R} m\right) \\
& \quad=c_{0} \hat{\otimes}_{R} \cdots \hat{\otimes}_{R} c_{k-1} \hat{\otimes}_{R} \Delta\left(c_{k}\right) \hat{\otimes}_{R} c_{k+1} \hat{\otimes}_{R} \cdots \hat{\otimes}_{R} c_{n-1} \hat{\otimes}_{R} m,
\end{aligned}
$$

while

$$
\begin{aligned}
& d^{n}\left(c_{0} \hat{\otimes}_{R} \cdots \hat{\otimes}_{R} c_{n-1} \hat{\otimes}_{R} m\right) \\
& \quad=c_{0\langle 2\rangle} \hat{\otimes}_{R} c_{1} \hat{\otimes}_{R} \cdots \hat{\otimes}_{R} c_{n-1} \hat{\otimes}_{R} c_{0\langle 1\rangle} m^{[-1]} \hat{\otimes}_{R} m^{[0]} .
\end{aligned}
$$

If $0 \leq k \leq n$, then the codegeneracy map $s^{k}$ is given by

$$
\begin{aligned}
& s^{k}\left(c_{0} \hat{\otimes}_{R} \cdots \hat{\otimes}_{R} c_{n+1} \hat{\otimes}_{R} m\right) \\
& \quad=c_{0} \hat{\otimes}_{R} \cdots \hat{\otimes}_{R} c_{k-1} \hat{\otimes}_{R} c_{k} \epsilon\left(c_{k+1}\right) \hat{\otimes}_{R} c_{k+2} \hat{\otimes}_{R} \cdots \hat{\otimes}_{R} c_{n+1} \hat{\otimes}_{R} m .
\end{aligned}
$$

The para-cocyclic map is

$$
t^{n}\left(c_{0} \hat{\otimes}_{R} \ldots \hat{\otimes}_{R} c_{n} \hat{\otimes}_{R} m\right)=c_{1} \hat{\otimes}_{R} \ldots \hat{\otimes}_{R} c_{n} \hat{\otimes}_{R} c_{0} m^{[-1]} \hat{\otimes}_{R} m^{[0]} .
$$

Restricting to the case when $B$ is a bialgebra over $k$, this para-cocyclic module yields a symmetrical version of [12], (2.1)-(2.4) (note the minor difference of using a left or a right module coalgebra). In [12] additional assumptions are made on the left comodule $M$ under which a truly cocyclic quotient exists.

Example 3.7. Let $B$ be a left bialgebroid over a $k$-algebra $L$ and $(A, \mu, \eta)$ be a left $B$-module algebra, hence in particular an $L$-ring. The following data define an object in $\mathcal{A}$ :

- The monads $T_{l}=\operatorname{Hom}_{-, L}(A,-)$ and $T_{r}=\operatorname{Hom}_{L,-}(A,-)$ on $A$-Mod- $A$. For any $A$-bimodule $X, T_{l} X=\operatorname{Hom}_{-, L}(A, X)$ is an $A$-bimodule via

$$
\left(a_{1} f a_{2}\right)(a)=a_{1} f\left(a_{2} a\right) \quad \text { for } a, a_{1}, a_{2} \in A, f \in \operatorname{Hom}_{-, L}(A, X) .
$$

The monad structure is given by

$$
\operatorname{Hom}_{-, L}\left(A \otimes_{L} \eta, X\right): T_{l}^{2} X \rightarrow T_{l} X \quad \text { and } \quad X \rightarrow T_{l} X, \quad x \mapsto[a \mapsto x a] .
$$


Symmetrically, $T_{r} X=\operatorname{Hom}_{L,-}(A, X)$ is an $A$-bimodule via

$$
\left(a_{1} g a_{2}\right)(a)=g\left(a a_{1}\right) a_{2} \quad \text { for } a, a_{1}, a_{2} \in A, g \in \operatorname{Hom}_{L,-}(A, X) .
$$

The monad structure is given by

$$
\operatorname{Hom}_{L,-}\left(\eta \otimes_{L} A, X\right): T_{r}^{2} X \rightarrow T_{r} X \quad \text { and } \quad X \rightarrow T_{r} X, \quad x \mapsto[a \mapsto a x] .
$$

- The distributive law

$$
\begin{aligned}
\Phi: \operatorname{Hom}_{-, L}\left(A, \operatorname{Hom}_{L,-}(A,-)\right) & \cong \operatorname{Hom}_{L, L}\left(A \otimes_{k} A,-\right) \\
& \cong \operatorname{Hom}_{L,-}\left(A, \operatorname{Hom}_{-, L}(A,-)\right) .
\end{aligned}
$$

- The right $\Phi$-module functor $\sqcap: A$-Mod- $A \rightarrow$ Mod- $k$ given for any $A$-bimodule $X$ by the equalizer

$$
\sqcap X \longrightarrow \operatorname{Hom}_{L, L}(L, X) \underset{f \mapsto[a \mapsto f(1) a]}{\stackrel{f \mapsto[a \mapsto a f(1)]}{\longrightarrow}} \operatorname{Hom}_{L, L}(A, X) .
$$

That is, $\sqcap X$ is the center of the $A$-bimodule $X$. The natural transformation $i$ is given by the isomorphism

$$
\sqcap \operatorname{Hom}_{-, L}(A,-) \cong \operatorname{Hom}_{L, L}(L,-) \cong \sqcap \operatorname{Hom}_{L,-}(A,-) .
$$

- The left $\Phi$-module functor $\sqcup=\operatorname{Hom}_{L,-}(A,-): B$-Ctrmod $\rightarrow A$-Mod- $A$. For any left $B$-contramodule $(Q, \alpha), \sqcup Q=\operatorname{Hom}_{L,-}(A, Q)$ is an $A$-bimodule via

$$
\left(a_{1} g a_{2}\right)(a)=\alpha\left(g\left(\left(-a_{2}\right) a a_{1}\right)\right) \quad \text { for } a, a_{1}, a_{2} \in A, g \in \operatorname{Hom}_{L,-}(A, Q) .
$$

The map $w: \operatorname{Hom}_{L,-}\left(A, \operatorname{Hom}_{L,-}(A,-)\right) \rightarrow \operatorname{Hom}_{-, L}\left(A, \operatorname{Hom}_{L,-}(A,-)\right)$ is given by

$$
\left(w_{Q}(h)\right)(a)(b)=\alpha(h(b)(-a)) \text { for } a, b \in A, h \in \operatorname{Hom}_{L,-}\left(A, \operatorname{Hom}_{L,-}(A, Q)\right) .
$$

For any left $B$-contramodule $(Q, \alpha)$, this determines a para-cocyclic object in Mod- $k$. It is given by $\sqcap \operatorname{Hom}_{-, L}\left(A^{\otimes_{L} n+1}, \operatorname{Hom}_{L,-}(A, Q)\right) \cong \operatorname{Hom}_{L, L}\left(A^{\otimes_{L} n+1}, Q\right)$ at degree $n$. For every $0 \leq k \leq n-1$, the corresponding coface map is

$$
\begin{aligned}
& \left(d^{k} \varphi^{(n-1)}\right)\left(a_{0} \otimes_{L} \cdots \otimes_{L} a_{n}\right) \\
& \quad=\varphi^{(n-1)}\left(a_{0} \otimes_{L} \cdots \otimes_{L} a_{k-1} \otimes_{L} a_{k} a_{k+1} \otimes_{L} a_{k+2} \otimes_{L} \cdots \otimes_{L} a_{n}\right),
\end{aligned}
$$

while

$$
\begin{aligned}
& \left(d^{n} \varphi^{(n-1)}\right)\left(a_{0} \otimes_{L} \cdots \otimes_{L} a_{n}\right) \\
& \quad=\alpha\left(\varphi^{(n-1)}\left(\left(-a_{n}\right) a_{0} \otimes_{L} a_{1} \otimes_{L} \cdots \otimes_{L} a_{n-1}\right)\right) .
\end{aligned}
$$


If $0 \leq k \leq n$, then the codegeneracy map $s^{k}$ is given by

$$
\begin{aligned}
& \left(s^{k} \varphi^{(n+1)}\right)\left(a_{0} \otimes_{L} \cdots \otimes_{L} a_{n}\right) \\
& \quad=\varphi^{(n+1)}\left(a_{0} \otimes_{L} \cdots \otimes_{L} a_{k} \otimes_{L} 1_{A} \otimes_{L} a_{k+1} \otimes_{L} \cdots \otimes_{L} a_{n}\right) .
\end{aligned}
$$

The para-cocyclic map is

$$
\left(t^{n} \varphi^{(n)}\right)\left(a_{0} \otimes_{L} \cdots \otimes_{L} a_{n}\right)=\alpha\left(\varphi^{(n)}\left(-a_{n} \otimes_{L} a_{0} \otimes_{L} \cdots \otimes_{L} a_{n-1}\right)\right),
$$

where $\varphi^{(j)} \in \operatorname{Hom}_{L, L}\left(A^{\otimes_{L} j+1}, Q\right)$.

This yields a non-commutative base version of the para-cocyclic module in [5], p. 6. (In [5] additional assumptions are made on the left contramodule $(Q, \alpha)$ under which a truly cocyclic subobject exists.)

Example 3.8. Let $B$ be a left bialgebroid over a $k$-algebra $L$ and $(A, \mu, \eta)$ be a right $B$-comodule algebra with $B$-coaction $a \mapsto a_{[0]} \otimes_{L} a_{[1]}$. Then $(A, \mu, \eta)$ is in particular an $R:=L^{\mathrm{op}}$-ring. The following data determine an object in $\mathcal{A}$ :

- The same monads $T_{l}=\operatorname{Hom}_{-, R}(A,-)$ and $T_{r}=\operatorname{Hom}_{R,-}(A,-)$ on $A$-Mod- $A$, introduced in Example 3.7 (replacing $L$ by $R$ ).

- The same distributive law $\Phi$, introduced in Example 3.7 (replacing $L$ by $R$ ).

- The same right $\Phi$-module functor $(\sqcap, i)$, introduced in Example 3.7 (replacing $L$ by $R$ );

- The left $\Phi$-module functor $\sqcup=\operatorname{Hom}_{R,-}(A,-): \operatorname{Mod}-B \rightarrow A$-Mod- $A$. For any right $B$-module $N, \sqcup N=\operatorname{Hom}_{R,-}(A, N)$ is an $A$-bimodule via

$$
\left(a_{1} g a_{2}\right)(a)=g\left(a_{2[0]} a a_{1}\right) a_{2[1]} \quad \text { for } a, a_{1}, a_{2} \in A, g \in \operatorname{Hom}_{R,-}(A, N) .
$$

The map $w: \operatorname{Hom}_{R,-}\left(A, \operatorname{Hom}_{R,-}(A,-)\right) \rightarrow \operatorname{Hom}_{-, R}\left(A, \operatorname{Hom}_{R,-}(A,-)\right)$ is given by

$$
\left(w_{N}(h)\right)(a)(b)=h(b)\left(a_{[0]}\right) a_{[1]} .
$$

For any right $B$-module $N$, this determines a para-cocyclic object in Mod- $k$. At degree $n$, it is given by $\sqcap \operatorname{Hom}_{-, R}\left(A^{\otimes_{R} n+1}, \operatorname{Hom}_{R,-}(A, N)\right) \cong \operatorname{Hom}_{R, R}\left(A^{\otimes_{R} n+1}, N\right)$. For every $0 \leq k \leq n-1$, the corresponding coface map is

$$
\begin{aligned}
& \left(d^{k} \varphi^{(n-1)}\right)\left(a_{0} \otimes_{R} \cdots \otimes_{R} a_{n}\right) \\
& \quad=\varphi^{(n-1)}\left(a_{0} \otimes_{R} \cdots \otimes_{R} a_{k-1} \otimes_{R} a_{k} a_{k+1} \otimes_{R} a_{k+2} \otimes_{R} \cdots \otimes_{R} a_{n}\right),
\end{aligned}
$$

while

$$
\left(d^{n} \varphi^{(n-1)}\right)\left(a_{0} \otimes_{R} \cdots \otimes_{R} a_{n}\right)=\varphi^{(n-1)}\left(a_{n[0]} a_{0} \otimes_{R} a_{1} \otimes_{R} \cdots \otimes_{R} a_{n-1}\right) a_{n[1]} .
$$

If $0 \leq k \leq n$, then the codegeneracy map $s^{k}$ is given by

$$
\begin{aligned}
& \left(s^{k} \varphi^{(n+1)}\right)\left(a_{0} \otimes_{R} \cdots \otimes_{R} a_{n}\right) \\
& \quad=\varphi^{(n+1)}\left(a_{0} \otimes_{R} \cdots \otimes_{R} a_{k} \otimes_{R} 1_{A} \otimes_{R} a_{k+1} \otimes_{R} \cdots \otimes_{R} a_{n}\right) .
\end{aligned}
$$


The para-cocyclic map is

$$
\left(t^{n} \varphi^{(n)}\right)\left(a_{0} \otimes_{R} \cdots \otimes_{R} a_{n}\right)=\varphi^{(n)}\left(a_{n[0]} \otimes_{R} a_{0} \otimes_{R} \cdots \otimes_{R} a_{n-1}\right) a_{n[1]},
$$

where $\varphi^{(j)} \in \operatorname{Hom}_{R, R}\left(A^{\otimes_{R} j+1}, N\right)$.

This yields a non-commutative base version of the para-cocyclic module in [12], (3.1)-(3.4). (In [12] additional assumptions are made on the right module $N$ under which a truly cocyclic submodule exists.)

\section{Para-cyclic objects}

Symmetrically to the considerations in Section 1 and Section 2, one can obtain another category $\mathcal{B}$ together with a functor $\mathcal{Z}_{*}$ from $\mathcal{B}$ to a category of para-cyclic objects in the category of functors. Without repeating the details, in this section we summarize the main steps.

Definition 4.1. A comonad on a category $\mathcal{M}$ is a triple $(S, d, e)$, where $S: \mathcal{M} \rightarrow \mathcal{M}$ is a functor and $d: S \rightarrow S^{2}$ and $e: S \rightarrow \mathcal{M}$ are natural transformations called the comultiplication and counit, respectively. Their string representations are shown in the first two pictures of the figure below. They satisfy the coassociativity and counitality constraints expressed by the third and the fourth equalities in the same figure.

A morphism from a comonad $\left(S^{\prime}, d^{\prime}, e^{\prime}\right)$ on $\mathcal{M}^{\prime}$ to a comonad $(S, d, e)$ on $\mathcal{M}$ is a pair $(F, f)$, where $F: \mathcal{M}^{\prime} \rightarrow \mathcal{M}$ is a functor and $f: S F \rightarrow F S^{\prime}$ is a natural transformation which is compatible with the comultiplications and the counits in the sense of the last two relations of the following figure.
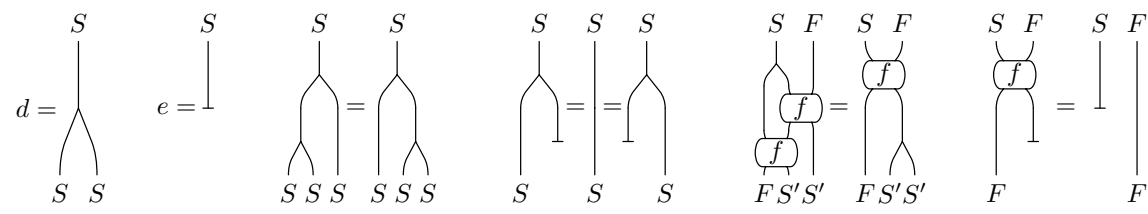

Comonads and their morphisms constitute a category which is isomorphic to the category of 2-functors and lax natural transformations, from the vertical opposite of the 2-category $\mathcal{T}$ in Section 1 to Cat.

Definition 4.2. A coalgebra for a comonad $S$ on a category $\mathcal{M}$ is a pair $(M, \varrho)$, where $M$ is an object in $\mathcal{M}$ and $\varrho: M \rightarrow S M$ is a morphism in $\mathcal{M}$ which is coassociative and counital in the evident sense.

A morphism of $S$-coalgebras $\left(M^{\prime}, \varrho^{\prime}\right) \rightarrow(M, \varrho)$ is a morphism $\varphi: M^{\prime} \rightarrow M$ in $\mathcal{M}$ such that $S \varphi \circ \varrho^{\prime}=\varrho \circ \varphi$. Coalgebras of a comonad $S$ and their morphisms constitute the so-called Eilenberg-Moore category $\mathcal{M}^{S}$. 
Via composition on the right, a comonad $S: \mathcal{M} \rightarrow \mathcal{M}$ induces a comonad Cat $(S,-)$ on the category Cat $(\mathcal{M},-)$. Symmetrically, there is a comonad Cat $(-, S)$ on the category Cat $(-, \mathcal{M})$. We call a coalgebra of the comonad $\operatorname{Cat}(S,-)$ a right $S$-comodule functor and we term a coalgebra of the comonad Cat $(-, S)$ a left $S$ comodule functor.

Definition 4.3. Consider two comonads $\left(S_{l}, d_{l}, e_{l}\right)$ and $\left(S_{r}, d_{r}, e_{r}\right)$ on the same category $\mathcal{M}$. A comonad distributive law is a natural transformation $\Psi: S_{l} S_{r} \rightarrow S_{r} S_{l}$ such that the following equalities hold.
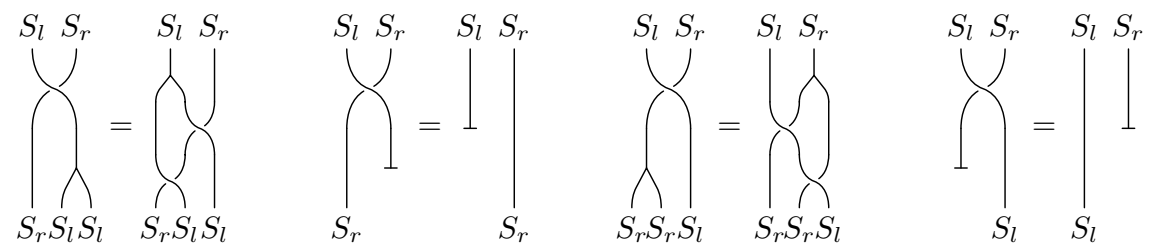

A comonad distributive law $\Psi: S_{l} S_{r} \rightarrow S_{r} S_{l}$ as in Definition 4.3 induces a comonad structure on the composite functor $S_{l} S_{r}$, with comultiplication $d$ and counit $e$ whose string representations are given in the figure below.
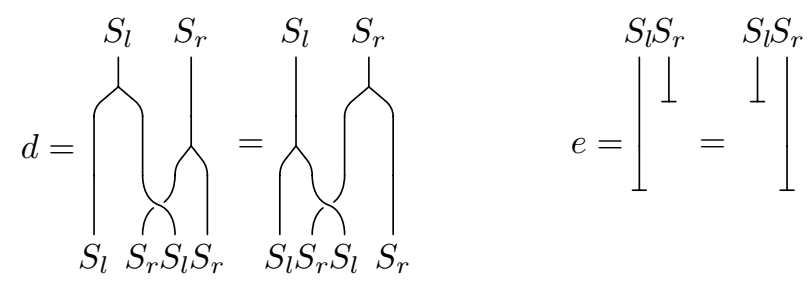

Definition 4.4. Consider two comonads $\left(S_{l}, d_{l}, e_{l}\right)$ and $\left(S_{r}, d_{r}, e_{r}\right)$ on the same category $\mathcal{M}$ and a comonad distributive law $\Psi: S_{l} S_{r} \rightarrow S_{r} S_{l}$. A $\Psi$-coalgebra is a pair consisting of an object $X$ in $\mathcal{M}$ and a morphism $\xi: S_{l} X \rightarrow S_{r} X$ rendering commutative the following diagrams.

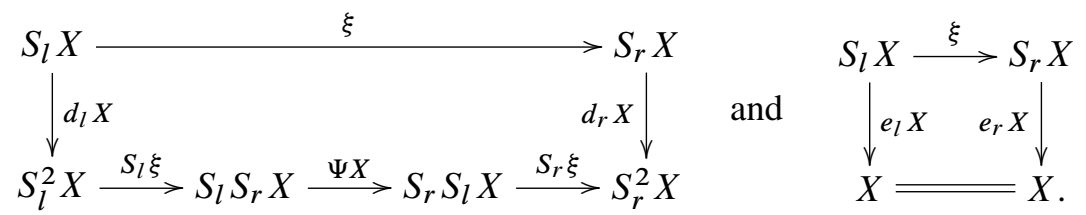

Coalgebras of the comonad distributive law $\operatorname{Cat}(\Psi,-)$ are called right $\Psi$-comodule functors. Consider two comonads $\left(S_{l}, d_{l}, e_{l}\right)$ and $\left(S_{r}, d_{r}, e_{r}\right)$ on the same category $\mathcal{M}$ and two comonads $\left(S_{l}^{\prime}, d_{l}^{\prime}, e_{l}^{\prime}\right)$ and $\left(S_{r}^{\prime}, d_{r}^{\prime}, e_{r}^{\prime}\right)$ on $\mathcal{M}^{\prime}$. Let $\Psi: S_{l} S_{r} \rightarrow S_{r} S_{l}$ and $\Psi^{\prime}: S_{l}^{\prime} S_{r}^{\prime} \rightarrow S_{r}^{\prime} S_{l}^{\prime}$ be comonad distributive laws. A morphism from a right $\Psi^{\prime}$ comodule functor $\left(\Pi^{\prime}: \mathcal{M}^{\prime} \rightarrow \mathcal{\ell}^{\prime}, i^{\prime}: \Pi^{\prime} S_{r}^{\prime} \rightarrow \Pi^{\prime} S_{l}^{\prime}\right)$ to a right $\Psi$-module functor 
$\left(\sqcap: \mathcal{M} \rightarrow \mathcal{C}, i: \sqcap S_{r} \rightarrow \sqcap S_{l}\right)$ is a quintuple $\left(G, q_{l}, q_{r}, \wedge, \pi\right)$, where $\wedge: \mathcal{C}^{\prime} \rightarrow \mathcal{C}$ is a functor, $\pi: \sqcap G \rightarrow \wedge \Pi^{\prime}$ is a natural transformation, $\left(G, q_{l}\right):\left(S_{l}^{\prime}, d_{l}^{\prime}, e_{l}^{\prime}\right) \rightarrow$ $\left(S_{l}, d_{l}, e_{l}\right)$ and $\left(G, q_{r}\right):\left(S_{r}^{\prime}, d_{r}^{\prime}, e_{r}^{\prime}\right) \rightarrow\left(S_{r}, d_{r}, e_{r}\right)$ are comonad morphisms. These data are, in addition, subject to the following two conditions.
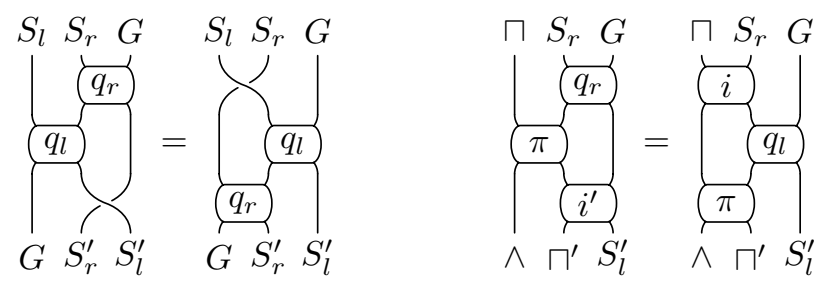

Right $\Psi$-comodule functors and their morphisms constitute a category which is isomorphic to the category of 2-functors and lax natural transformations, from the vertical opposite of the 2-category $\mathcal{R}$ in Section 1 to Cat.

Symmetrically, a left $\Psi$-comodule functor is a coalgebra for the comonad distributive law Cat $(-, \Psi)$. A morphism from a left $\Psi^{\prime}$-module functor $\left(\sqcup^{\prime}: \mathscr{D}^{\prime} \rightarrow\right.$ $\left.\mathcal{M}^{\prime}, w^{\prime}: S_{l}^{\prime} \sqcup^{\prime} \rightarrow S_{r}^{\prime} \sqcup^{\prime}\right)$ to a left $\Psi$-module functor $\left(\sqcup: \mathscr{D} \rightarrow \mathcal{M}, w: S_{l} \sqcup \rightarrow\right.$ $\left.S_{r} \sqcup\right)$ is a quintuple $\left(G, q_{l}, q_{r}, \vee, \omega\right)$, where $\left(G, q_{l}\right):\left(S_{l}^{\prime}, d_{l}^{\prime}, e_{l}^{\prime}\right) \rightarrow\left(S_{l}, d_{l}, e_{l}\right)$ and $\left(G, q_{r}\right):\left(S_{r}^{\prime}, d_{r}^{\prime}, e_{r}^{\prime}\right) \rightarrow\left(S_{r}, d_{r}, e_{r}\right)$ are comonad morphisms such that the first condition in (4.2) holds, $\vee: \mathscr{D}^{\prime} \rightarrow \mathscr{D}$ is a functor and $\omega: \sqcup \vee \rightarrow G \sqcup^{\prime}$ is a natural transformation such that the following relation holds.

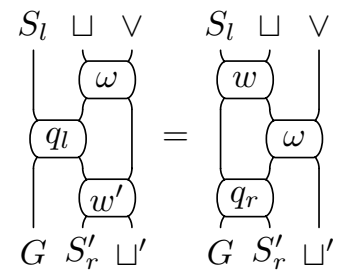

Left $\Psi$-comodule functors and their morphisms constitute a category which is isomorphic to the category of 2-functors and lax natural transformations, from the horizontal and vertical opposite of the 2-category $\mathcal{R}$ in Section 1 to Cat.

We can define a category $\mathcal{B}$ as the opposite of the category of 2-functors and lax natural transformations, from the vertical opposite of the 2-category $S$ in Section 1 to Cat:

Definition 4.5. The category $\mathscr{B}$ is defined to have objects $\left(S_{l}, S_{r}, \Psi, \sqcap, i, \sqcup, w\right)$, where

- $S_{l}$ and $S_{r}$ are comonads on the same category $\mathcal{M}$;

- $\Psi: S_{l} S_{r} \rightarrow S_{r} S_{l}$ is a comonad distributive law;

- $\left(\sqcap: \mathcal{M} \rightarrow \mathcal{C}, i: \sqcap S_{r} \rightarrow \sqcap S_{l}\right)$ is a right $\Psi$-comodule functor;

- $\left(\sqcup: \mathscr{D} \rightarrow \mathcal{M}, w: S_{l} \sqcup \rightarrow S_{r} \sqcup\right)$ is a left $\Psi$-comodule functor. 
A morphism $\left(S_{l}, S_{r}, \Psi, \sqcap, i, \sqcup, w\right) \rightarrow\left(S_{l}^{\prime}, S_{r}^{\prime}, \Psi^{\prime}, \Pi^{\prime}, i^{\prime}, \sqcup^{\prime}, w^{\prime}\right)$ is a datum $\left(G, q_{l}, q_{r}, \wedge, \pi, \vee, \omega\right)$ such that

- $\left(G, q_{l}, q_{r}, \wedge, \pi\right)$ is a morphism from the right $\Psi^{\prime}$-comodule functor $\left(\Pi^{\prime}, i^{\prime}\right)$ to the right $\Psi$-comodule functor $(\sqcap, i)$;

- $\left(G, q_{l}, q_{r}, \vee, \omega\right)$ is a morphism from the left $\Psi^{\prime}$-comodule functor $\left(\sqcup^{\prime}, w^{\prime}\right)$ to the left $\Psi$-comodule functor $(\sqcup, w)$.

Recall that the opposite $\mathcal{C}^{\mathrm{op}}$ of a category $\mathcal{C}$ has the same objects and morphisms as $\mathcal{C}$, but composition of morphisms is opposite to that in $\mathcal{C}$. A para-cyclic object in a category $\mathcal{C}$ is, by definition, a para-cocyclic object in $\ell^{\text {op }}$.

Definition 4.6. Objects of the category $\mathcal{P}$ are para-cyclic objects in the category of functors. Morphisms from $\left(Z_{*}: \mathscr{D} \rightarrow \mathcal{C}, d_{*}, s_{*}, t_{*}\right)$ to $\left(Z_{*}^{\prime}: \mathscr{D}^{\prime} \rightarrow \mathcal{C}^{\prime}, d_{*}^{\prime}, s_{*}^{\prime}, t_{*}^{\prime}\right)$ are triples $\left(\wedge, \vee, \xi_{*}\right)$, where $\wedge: \mathscr{C}^{\prime} \rightarrow \mathcal{C}^{\prime}$ and $\vee: \mathscr{D}^{\prime} \rightarrow \mathscr{D}$ are functors and $\xi_{*}:\left(Z_{*} \vee, d_{*} \vee, s_{*} \vee, t_{*} \vee\right) \rightarrow\left(\wedge Z_{*}^{\prime}, \wedge d_{*}^{\prime}, \wedge s_{*}^{\prime}, \wedge t_{*}^{\prime}\right)$ is a morphism of para-cyclic objects.

Dually to Theorem 2.3 the following holds.

Theorem 4.7. There is a functor $\mathcal{Z}_{*}: \mathscr{B} \rightarrow \underline{\mathcal{P}}$, with object map

$$
\left(S_{l}, S_{r}, \Psi, \sqcap: \mathcal{M} \rightarrow \mathcal{\ell}, i, \sqcup: \mathscr{D} \rightarrow \mathcal{M}, w\right) \mapsto\left(\sqcap S_{l}^{*+1} \sqcup: \mathscr{D} \rightarrow \mathcal{C}, d_{*}, s_{*}, t_{*}\right) .
$$

The functor $\mathcal{Z}_{*}$ takes a morphism

$$
\left(G, q_{l}, q_{r}, \wedge, \pi, \vee, \omega\right):\left(S_{l}, S_{r}, \Psi, \sqcap, i, \sqcup, w\right) \rightarrow\left(S_{l}^{\prime}, S_{r}^{\prime}, \Psi^{\prime}, \sqcap^{\prime}, i^{\prime}, \sqcup^{\prime}, w^{\prime}\right)
$$

to the triple $\left(\wedge, \vee, \xi_{*}\right)$. At every degree $n \geq 0$ and for $0 \leq k \leq n$, the face morphisms $d_{k}$, the degeneracy morphisms $s_{k}$, the para-cyclic morphism $t_{n}$ and the morphism $\xi_{n}$ are given by the natural transformations below.
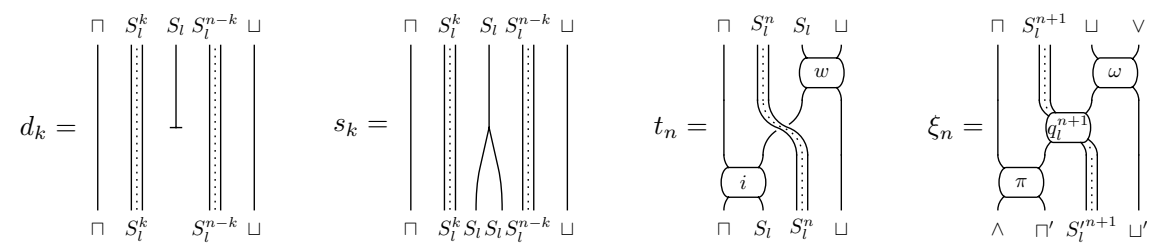

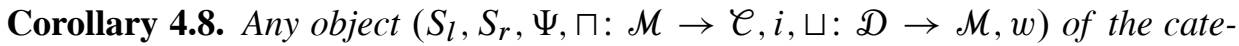
gory $\mathcal{B}$ determines a functor from $\mathcal{D}$ to the category of para-cyclic objects in $\mathcal{C}$. The objects of $\mathfrak{D}$ play the role of coefficients for the resulting para-cyclic object in $\mathcal{C}$. 


\section{Examples from Hopf cyclic theory}

In this section we present several examples of objects in the category $\mathcal{B}$ in Definition 4.5, similar to those we have seen in Section 3. Throughout this section the same notational conventions are used as in Section 3.

The first example is obtained from [4], Theorem 2.9 (for the case of a bialgebra see [17], Section 5.3).

Example 5.1. Let $B$ be a left bialgebroid over a $k$-algebra $L$ and $(C, \Delta, \epsilon)$ be a left $B$-comodule coring (hence in particular an $L$-coring), with $B$-coaction $c \mapsto$ $c_{[-1]} \otimes_{L} c_{[0]}$. An object in the category $\mathscr{B}$ is given by the following data:

- the comonads $S_{l}=C \otimes_{L}(-)$ and $S_{r}=(-) \otimes_{L} C$ on $L$-Mod- $L$, with comonad structures

$$
\Delta \otimes_{L}(-): S_{l} \rightarrow S_{l}^{2}, \quad \epsilon \otimes_{L}(-): S_{l} \rightarrow L-\operatorname{Mod}-L
$$

and

$$
(-) \otimes_{L} \Delta: S_{r} \rightarrow S_{r}^{2}, \quad(-) \otimes_{L} \epsilon: S_{r} \rightarrow L-\operatorname{Mod}-L,
$$

respectively;

- the trivial comonad distributive law $\Psi=C \otimes_{L}(-) \otimes_{L} C$;

- the right $\Psi$-comodule functor $(\sqcap, i)$, where $\Pi=L \otimes_{L^{\mathrm{e}}}(-): L$-Mod- $L \rightarrow$ Mod$k$ and for any $L$-bimodule $P$,

$$
i_{P}: P \hat{\otimes}_{L} C \rightarrow C \hat{\otimes}_{L} P, \quad p \hat{\otimes}_{L} c \mapsto c \hat{\otimes}_{L} p ;
$$

- the left $\Psi$-comodule functor $(\sqcup, w)$, where $\sqcup: B$-Mod $\rightarrow L$-Mod- $L$ is the forgetful functor and for any left $B$-module $N$,

$$
w_{N}: C \otimes_{L} N \rightarrow N \otimes_{L} C, \quad c \otimes_{L} m \mapsto c_{[-1]} m \otimes_{L} c_{[0]} .
$$

For any left $B$-module $N$, the corresponding para-cyclic object in Mod- $k$ is given by $C^{\hat{\otimes}_{L} n+1} \hat{\otimes}_{L} N$ at degree $n$. Face and degeneracy maps are, for $k=0, \ldots, n$,

$$
\begin{aligned}
& d_{k}\left(c_{0} \hat{\otimes}_{L} c_{1} \hat{\otimes}_{L} \cdots \hat{\otimes}_{L} c_{n} \hat{\otimes}_{L} m\right) \\
& \quad=c_{0} \hat{\otimes}_{L} c_{1} \hat{\otimes}_{L} \cdots \hat{\otimes}_{L} c_{k-1} \hat{\otimes}_{L} \epsilon\left(c_{k}\right) \hat{\otimes}_{L} c_{k+1} \hat{\otimes}_{L} \cdots \hat{\otimes}_{L} c_{n} \hat{\otimes}_{L} m \\
& s_{k}\left(c_{0} \hat{\otimes}_{L} c_{1} \hat{\otimes}_{L} \cdots \hat{\otimes}_{L} c_{n} \hat{\otimes}_{L} m\right) \\
& \quad=c_{0} \hat{\otimes}_{L} c_{1} \hat{\otimes}_{L} \cdots \hat{\otimes}_{L} c_{k-1} \hat{\otimes}_{L} \Delta\left(c_{k}\right) \hat{\otimes}_{L} c_{k+1} \hat{\otimes}_{L} \cdots \hat{\otimes}_{L} c_{n} \hat{\otimes}_{L} m .
\end{aligned}
$$

The para-cyclic map comes out as

$$
t_{n}\left(c_{0} \hat{\otimes}_{L} c_{1} \hat{\otimes}_{L} \cdots \hat{\otimes}_{L} c_{n} \hat{\otimes}_{L} m\right)=c_{n[0]} \hat{\otimes}_{L} c_{0} \hat{\otimes}_{L} \cdots \hat{\otimes}_{L} c_{n-1} \hat{\otimes}_{L} c_{n[-1]} m .
$$


A next example arises from Theorem 2.11 of [4]. Restricting it to the case of a bialgebra, it yields a symmetrical version of the para-cocyclic module in [17], Section 5.1.

Example 5.2. Let $B$ be a right bialgebroid over a $k$-algebra $R$ and $(C, \Delta, \epsilon)$ be a right $B$-module coring (so in particular an $R$-coring). An object in $\mathcal{B}$ is given by the following data:

- the same comonads $S_{l}=C \otimes_{R}(-)$ and $S_{r}=(-) \otimes_{R} C$ on $R$-Mod- $R$, introduced in Example 5.1 (replacing $L$ by $R$ );

- the trivial comonad distributive law $\Psi=C \otimes_{R}(-) \otimes_{R} C$;

- the same right $\Psi$-comodule functor $(\sqcap, i)$, introduced in Example 5.1 (replacing $L$ by $R$ );

- the left $\Psi$-comodule functor $(\sqcup, w)$, where $\sqcup:$ Comod- $B \rightarrow R$-Mod- $R$ is the forgetful functor and for any right $B$-comodule $M$, with coaction denoted by $m \mapsto m^{[0]} \otimes_{R} m^{[1]}$,

$$
w_{M}: C \otimes_{R} M \rightarrow M \otimes_{R} C, \quad c \otimes_{R} m \mapsto m^{[0]} \otimes_{R} c m^{[1]} .
$$

For any right $B$-comodule $M$, the corresponding para-cyclic module has the same simplicial structure as in Example 5.1 (replacing $L$ by $R$ and $N$ by $M$ ). The paracyclic map is

$t_{n}\left(c_{0} \hat{\otimes}_{R} c_{1} \hat{\otimes}_{R} \ldots \hat{\otimes}_{R} c_{n} \hat{\otimes}_{R} m\right)=c_{n} m^{[1]} \hat{\otimes}_{R} c_{0} \hat{\otimes}_{R} c_{1} \hat{\otimes}_{R} \ldots \hat{\otimes}_{R} c_{n-1} \hat{\otimes}_{R} m^{[0]}$.

Example 5.3. Let $B$ be a left bialgebroid over a $k$-algebra $L$ and $(A, \mu, \eta)$ be a left $B$-module algebra (so in particular an $L$-ring). An object in $B$ is given by the following data:

- the comonads $S_{l}=\operatorname{Hom}_{-, L}(A,-)$ and $S_{r}=\operatorname{Hom}_{L,-}(A,-)$ on $L-\operatorname{Mod}-L$, with comonad structures

$$
\operatorname{Hom}_{-, L}(\mu,-): S_{l} \rightarrow S_{l}^{2}, \quad \operatorname{Hom}_{-, L}(\eta,-): S_{l} \rightarrow L-\operatorname{Mod}-L
$$

and

$$
\operatorname{Hom}_{L,-}(\mu,-): S_{r} \rightarrow S_{r}^{2}, \quad \operatorname{Hom}_{L,-}(\eta,-): S_{r} \rightarrow L-\operatorname{Mod}-L,
$$

respectively;

- the comonad distributive law

$$
\begin{aligned}
\Psi: \operatorname{Hom}_{-, L}\left(A, \operatorname{Hom}_{L,-}(A,-)\right) & \cong \operatorname{Hom}_{L, L}\left(A \otimes_{k} A,-\right) \\
& \cong \operatorname{Hom}_{L,-}\left(A, \operatorname{Hom}_{-, L}(A,-)\right),
\end{aligned}
$$

given by switching the arguments; 
- the right $\Psi$-comodule functor $(\sqcap, i)$, where $\sqcap=\operatorname{Hom}_{L, L}(L,-): L$-Mod- $L \rightarrow$ Mod- $k$ and the natural transformation

$$
\begin{aligned}
i: \operatorname{Hom}_{L, L}\left(L, \operatorname{Hom}_{-, L}(A,-)\right) & \cong \operatorname{Hom}_{L, L}(A,-) \\
& \cong \operatorname{Hom}_{L, L}\left(L, \operatorname{Hom}_{L,-}(A,-)\right)
\end{aligned}
$$

is given by the hom-tensor adjunction isomorphisms;

- the left $\Psi$-comodule functor $(\sqcup, w)$, where $\sqcup:$ Ctrmod- $B \rightarrow L$-Mod- $L$ is the forgetful functor and for any right $B$-contramodule $(Q, \alpha)$,

$$
w_{Q}: \operatorname{Hom}_{-, L}(A, Q) \rightarrow \operatorname{Hom}_{L,-}(A, Q), \quad f \mapsto(a \mapsto \alpha(f(-a))) .
$$

The para-cyclic module corresponding to a right $B$-contramodule $(Q, \alpha)$ is given at degree $n$ by the $k$-module $\operatorname{Hom}_{L, L}\left(A^{\otimes_{L} n+1}, Q\right)$. Face and degeneracy maps are, for $k=0, \ldots, n$,

$$
\begin{aligned}
& \left(d_{k} \varphi^{(n)}\right)\left(a_{0} \otimes_{L} a_{1} \otimes_{L} \cdots \otimes_{L} a_{n-1}\right) \\
& \quad=\varphi^{(n)}\left(a_{0} \otimes_{L} \cdots \otimes_{L} a_{k-1} \otimes_{L} 1_{A} \otimes_{L} a_{k} \otimes_{L} \cdots \otimes_{L} a_{n-1}\right) \\
& \left(s_{k} \varphi^{(n)}\right)\left(a_{0} \otimes_{L} a_{1} \otimes_{L} \cdots \otimes_{L} a_{n+1}\right) \\
& \quad=\varphi^{(n)}\left(a_{0} \otimes_{L} \cdots \otimes_{L} a_{k-1} \otimes_{L} a_{k} a_{k+1} \otimes_{L} a_{k+2} \otimes_{L} \cdots \otimes_{L} a_{n+1}\right) .
\end{aligned}
$$

The para-cyclic map is

$$
\left(t_{n} \varphi^{(n)}\right)\left(a_{0} \otimes_{L} a_{1} \otimes_{L} \cdots \otimes_{L} a_{n}\right)=\alpha\left(\varphi^{(n)}\left(a_{1} \otimes_{L} \cdots \otimes_{L} a_{n} \otimes_{L}(-) a_{0}\right)\right)
$$

for $\varphi^{(n)} \in \operatorname{Hom}_{L, L}\left(A^{\otimes_{L} n+1}, Q\right)$.

Example 5.4. Let $B$ be a right bialgebroid over a $k$-algebra $R$ and $(A, \mu, \eta)$ be a right $B$-comodule algebra (so in particular an $R$-ring), with $B$-coaction $a \mapsto a^{[0]} \otimes_{R} a^{[1]}$. An object in $\mathcal{B}$ is given by the following data:

- the same comonads $S_{l}=\operatorname{Hom}_{-, R}(A,-)$ and $S_{r}=\operatorname{Hom}_{R,-}(A,-)$ on $R$-Mod$R$ as constructed in Example 5.3 (replacing $L$ by $R$ );

- the same comonad distributive law $\Psi$, constructed in Example 5.3 (replacing $L$ by $R)$;

- the same right $\Psi$-comodule functor $(\sqcap, i)$, constructed in Example 5.3 (replacing $L$ by $R$ );

- the left $\Psi$-comodule functor $(\sqcup, w)$, where $\sqcup: B$-Mod $\rightarrow R$-Mod- $R$ is the forgetful functor and for any left $B$-module $N$,

$$
w_{N}: \operatorname{Hom}_{-, R}(A, N) \rightarrow \operatorname{Hom}_{R,-}(A, N), \quad f \mapsto\left(a \mapsto a^{[1]} f\left(a^{[0]}\right)\right) .
$$

The para-cyclic module corresponding to a left $B$-module $N$ has the same simplicial structure as in Example 5.3 (replacing $L$ by $R$ and $Q$ by $N$ ). The para-cyclic map is

$$
\left(t_{n} \varphi^{(n)}\right)\left(a_{0} \otimes_{R} a_{1} \otimes_{R} \cdots \otimes_{R} a_{n}\right)=a_{0}^{[1]} \varphi^{(n)}\left(a_{1} \otimes_{R} \cdots \otimes_{R} a_{n} \otimes_{R} a_{0}^{[0]}\right) .
$$


Example 5.5. Let $B$ be a left bialgebroid over a $k$-algebra $L$ and $(A, \mu, \eta)$ be a left $B$-module algebra (so in particular an $L$-ring). An object in $\mathscr{B}$ is given by the following data.

- The comonads $S_{l}=A \otimes_{L}(-)$ and $S_{r}=(-) \otimes_{L} A$ on $A$-Mod- $A$. For any $A$-bimodule $X, S_{l} X=A \otimes_{L} X$ is an $A$-bimodule via

$$
a_{1}\left(a \otimes_{L} x\right) a_{2}=a_{1} a \otimes_{L} x a_{2} \text { for } a, a_{1}, a_{2} \in A, x \in X .
$$

The comonad structure is given by

$A \otimes_{L} \eta \otimes_{L} X: S_{l} X \rightarrow S_{l}^{2} X \quad$ and $\quad S_{l} X \rightarrow X, \quad a \otimes_{L} x \mapsto a x$.

Symmetrically, $S_{r} X=X \otimes_{L} A$ is an $A$-bimodule via

$$
a_{1}\left(x \otimes_{L} a\right) a_{2}=a_{1} x \otimes_{L} a a_{2} \quad \text { for } a, a_{1}, a_{2} \in A, x \in X .
$$

The comonad structure is given by

$$
X \otimes_{L} \eta \otimes_{L} A: S_{r} X \rightarrow S_{r}^{2} X \quad \text { and } \quad S_{r} X \rightarrow X, \quad x \otimes_{L} a \mapsto x a .
$$

- The trivial comonad distributive law $\Psi=A \otimes_{L}(-) \otimes_{L} A$.

- The right $\Psi$-comodule functor $\sqcap: A \hat{\otimes}_{A}(-): A$-Mod- $A \rightarrow \operatorname{Mod}-k$. The natural transformation $i$ is given by the isomorphism

$$
i: A \hat{\otimes}_{A}\left((-) \otimes_{L} A\right) \cong L \hat{\otimes}_{L}(-) \cong A \hat{\otimes}_{A}\left(A \otimes_{L}(-)\right) .
$$

- The left $\Psi$-comodule functor $\sqcup=(-) \otimes_{L} A$ : Comod- $B \rightarrow A$-Mod- $A$. For any right $B$-comodule $M$, with coaction $m \mapsto m_{[0]} \otimes_{L} m_{[1]}, \sqcup M=M \otimes_{L} A$ is an $A$-bimodule via

$$
a_{1}\left(m \otimes_{L} a\right) a_{2}=m_{[0]} \otimes_{L}\left(m_{[1]} a_{1}\right) a a_{2} \quad \text { for } a, a_{1}, a_{2} \in A, m \in M .
$$

The natural transformation $w: A \otimes_{L}(-) \otimes_{L} A \rightarrow(-) \otimes_{L} A \otimes_{L} A$ is given by

$$
w_{M}\left(a \otimes_{L} m \otimes_{L} b\right)=m_{[0]} \otimes_{L} m_{[1]} a \otimes_{L} b .
$$

For any right $B$-comodule $M$, this determines a para-cyclic object in Mod- $k$. At degree $n$ it is given by $A \hat{\otimes}_{A}\left(A^{\otimes_{L} n+1} \otimes_{L} M \otimes_{L} A\right) \cong A^{\otimes_{L}}{ }^{n+1} \widehat{\otimes}_{L} M$. For every $0 \leq k \leq n-1$, the corresponding face map is

$$
\begin{aligned}
& d_{k}\left(a_{0} \hat{\otimes}_{L} \ldots \hat{\otimes}_{L} a_{n} \hat{\otimes}_{L} m\right) \\
& \quad=a_{0} \hat{\otimes}_{L} \ldots \hat{\otimes}_{L} a_{k-1} \hat{\otimes}_{L} a_{k} a_{k+1} \hat{\otimes}_{L} a_{k+2} \hat{\otimes}_{L} \ldots \hat{\otimes}_{L} a_{n} \hat{\otimes}_{L} m,
\end{aligned}
$$

while

$$
\begin{aligned}
& d_{n}\left(a_{0} \hat{\otimes}_{L} \ldots \hat{\otimes}_{L} a_{n} \hat{\otimes}_{L} m\right) \\
& \quad=\left(m_{[1]} a_{n}\right) a_{0} \hat{\otimes}_{L} a_{1} \hat{\otimes}_{L} \ldots \hat{\otimes}_{L} a_{n-1} \hat{\otimes}_{L} m_{[0]} .
\end{aligned}
$$


If $0 \leq k \leq n$, then the degeneracy map $s_{k}$ is given by

$$
\begin{aligned}
& s_{k}\left(a_{0} \hat{\otimes}_{L} \cdots \hat{\otimes}_{L} a_{n} \hat{\otimes}_{L} m\right) \\
& \quad=a_{0} \hat{\otimes}_{L} \cdots \hat{\otimes}_{L} a_{k} \hat{\otimes}_{L} 1_{A} \hat{\otimes}_{L} a_{k+1} \hat{\otimes}_{L} \cdots \hat{\otimes}_{L} a_{n} \hat{\otimes}_{L} m .
\end{aligned}
$$

The para-cyclic map is

$$
t_{n}\left(a_{0} \hat{\otimes}_{L} \ldots \hat{\otimes}_{L} a_{n} \hat{\otimes}_{L} m\right)=m_{[1]} a_{n} \hat{\otimes}_{L} a_{0} \hat{\otimes}_{L} \ldots \hat{\otimes}_{L} a_{n-1} \hat{\otimes}_{L} m_{[0]} .
$$

If the left bialgebroid $B$ above is a constituent left bialgebroid in a Hopf algebroid with a bijective antipode $S$, then there is a bijective correspondence between right $B$ comodules $M$, with coaction $m \mapsto m_{[0]} \otimes_{L} m_{[1]}$, and left coactions of the constituent right bialgebroid of the Hopf algebroid on $M, m \mapsto S\left(m_{[1]}\right) \otimes_{L^{\text {op }}} m_{[0]}$, cf. A.10. Expressing in the above formulae the right $B$-coaction on $M$ in terms of this left coaction, we obtain a para-cyclic module. Restricting to the case of a Hopf algebra (instead of a Hopf algebroid) and applying to the obtained para-cyclic module the functor $\operatorname{Hom}_{k}(-, k)$, we obtain the para-cyclic module in [12], (2.6)-(2.9). (Note that in [12] further properties of the comodule $M$ are assumed which ensure that the associated para-cyclic module has a truly cyclic subobject.)

Example 5.6. Let $B$ be a left bialgebroid over a $k$-algebra $L$ and $(A, \mu, \eta)$ be a right $B$-comodule algebra with $B$-coaction $a \mapsto a_{[0]} \otimes_{L} a_{[1]}$. Then $(A, \mu, \eta)$ is in particular an $R:=L^{\mathrm{op}}$-ring. An object in $\mathscr{B}$ is given by the following data.

- The same comonads $S_{l}=A \otimes_{R}(-)$ and $S_{r}=(-) \otimes_{R} A$ on $A$-Mod- $A$ as constructed in Example 5.5 (replacing $L$ by $R$ ).

- The same comonad distributive law $\Psi$ as in Example 5.5 (replacing $L$ by $R$ ).

- The same right $\Psi$-comodule functor $(\sqcap, i)$ as in Example 5.5 (replacing $L$ by $R)$.

- The left $\Psi$-comodule functor $\sqcup=(-) \otimes_{R} A: B$-Mod $\rightarrow A$-Mod- $A$. For any left $B$-module $N, \sqcup N=N \otimes_{R} A$ is an $A$-bimodule via

$$
a_{1}\left(m \otimes_{R} a\right) a_{2}=a_{1[1]} m \otimes_{R} a_{1[0]} a a_{2} \quad \text { for } a, a_{1}, a_{2} \in A, m \in N .
$$

The natural transformation $w: A \otimes_{R}(-) \otimes_{R} A \rightarrow(-) \otimes_{R} A \otimes_{R} A$ is given by

$$
w_{N}\left(a \otimes_{R} m \otimes_{R} b\right)=a_{[1]} m \otimes_{R} a_{[0]} \otimes_{R} b .
$$

For any left $B$-module $N$, this determines a para-cyclic object in Mod- $k$. At degree $n$ it is given by $A \hat{\otimes}_{A}\left(A^{\otimes_{R} n+1} \otimes_{R} N \otimes_{R} A\right) \cong A^{\otimes_{R} n+1} \widehat{\otimes}_{R} N$. For every $0 \leq k \leq n-1$, the corresponding face map is

$$
\begin{aligned}
d_{k}\left(a_{0}\right. & \left.\hat{\otimes}_{R} \ldots \hat{\otimes}_{R} a_{n} \hat{\otimes}_{R} m\right) \\
& =a_{0} \hat{\otimes}_{R} \ldots \hat{\otimes}_{R} a_{k-1} \hat{\otimes}_{R} a_{k} a_{k+1} \hat{\otimes}_{R} a_{k+2} \hat{\otimes}_{R} \ldots \hat{\otimes}_{R} a_{n} \hat{\otimes}_{R} m,
\end{aligned}
$$


while

$$
\begin{aligned}
& d_{n}\left(a_{0} \hat{\otimes}_{R} \ldots \hat{\otimes}_{R} a_{n} \hat{\otimes}_{R} m\right) \\
& \quad=a_{n[0]} a_{0} \hat{\otimes}_{R} a_{1} \hat{\otimes}_{R} \ldots \hat{\otimes}_{R} a_{n-1} \hat{\otimes}_{R} a_{n[1]} m .
\end{aligned}
$$

If $0 \leq k \leq n$, then the degeneracy map $s_{k}$ is given by

$$
\begin{aligned}
& s_{k}\left(a_{0} \hat{\otimes}_{R} \ldots \hat{\otimes}_{R} a_{n} \hat{\otimes}_{R} m\right) \\
& \quad=a_{0} \hat{\otimes}_{R} \ldots \hat{\otimes}_{R} a_{k} \hat{\otimes}_{R} 1_{A} \hat{\otimes}_{R} a_{k+1} \hat{\otimes}_{R} \ldots \hat{\otimes}_{R} a_{n} \hat{\otimes}_{R} m .
\end{aligned}
$$

The para-cyclic map is

$$
t_{n}\left(a_{0} \hat{\otimes}_{R} \ldots \hat{\otimes}_{R} a_{n} \hat{\otimes}_{R} m\right)=a_{n[0]} \hat{\otimes}_{R} a_{0} \hat{\otimes}_{R} \ldots \hat{\otimes}_{R} a_{n-1} \hat{\otimes}_{R} a_{n[1]} m .
$$

This is a non-commutative base version of the para-cyclic module in [12], (3.5)-(3.8). Note that in [12] further properties of the left module $N$ are assumed which ensure that the associated para-cyclic module has a truly cyclic subobject.

A bicontramodule of an $R$-coring $C$ is an $R$-bimodule $Y$, together with a right $C$ contramodule structure $\beta_{r}$ : $\operatorname{Hom}_{-, R}(C, Y) \rightarrow Y$ and a left $C$-contramodule structure $\beta_{l}: \operatorname{Hom}_{R,-}(C, Y) \rightarrow Y$ such that $\beta_{r}$ is a left $R$-module map, $\beta_{l}$ is a right $R$-module map and

$$
\beta_{l} \circ \operatorname{Hom}_{R,-}\left(C, \beta_{r}\right)=\beta_{r} \circ \operatorname{Hom}_{-, R}\left(C, \beta_{l}\right),
$$

up to the (suppressed) canonical isomorphism

$$
\begin{aligned}
\operatorname{Hom}_{R,-}\left(C, \operatorname{Hom}_{-, R}(C, Y)\right) & \cong \operatorname{Hom}_{R, R}\left(C \otimes_{k} C, Y\right) \\
& \cong \operatorname{Hom}_{-, R}\left(C, \operatorname{Hom}_{R,-}(C, Y)\right) .
\end{aligned}
$$

A morphism of bicontramodules is a right contramodule map as well as a left contramodule map. The category of $C$-bicontramodules is denoted by $C$-Ctrmod- $C$.

Example 5.7. Let $B$ be a right bialgebroid over a $k$-algebra $R$ and $(C, \Delta, \epsilon)$ be a right $B$-module coring (hence in particular an $R$-coring). An object in $\mathcal{B}$ is given by the following data.

- The comonads $S_{l}=\operatorname{Hom}_{-, R}(C,-)$ and $S_{r}=\operatorname{Hom}_{R,-}(C,-)$ on $C$-Ctrmod- $C$. For any $C$-bicontramodule $\left(Y, \beta_{l}, \beta_{r}\right), S_{l} Y=\operatorname{Hom}_{-, R}(C, Y)$ is a bicontramodule, via the structure maps

$$
\begin{aligned}
& \operatorname{Hom}_{R,-}\left(C, \operatorname{Hom}_{-, R}(C, Y)\right) \\
& \quad \cong \operatorname{Hom}_{-, R}\left(C, \operatorname{Hom}_{R,-}(C, Y)\right) \stackrel{\operatorname{Hom}_{-, R}\left(C, \beta_{l}\right)}{\longrightarrow} \operatorname{Hom}_{-, R}(C, Y), \\
& \operatorname{Hom}_{-, R}\left(C, \operatorname{Hom}_{-, R}(C, Y)\right) \\
& \left.\quad \cong \operatorname{Hom}_{-, R}\left(C \otimes_{R} C, Y\right)\right) \stackrel{\operatorname{Hom}_{-, R}(\Delta, Y)}{\longrightarrow} \operatorname{Hom}_{-, R}(C, Y) .
\end{aligned}
$$


The comonad structure is given by

$$
\operatorname{Hom}_{-, R}\left(C \otimes_{R} \epsilon, Y\right): S_{l} Y \rightarrow S_{l}^{2} Y \quad \text { and } \quad \beta_{r}: S_{l} Y \rightarrow Y .
$$

Symmetrically, $S_{r} Y=\operatorname{Hom}_{R,-}(C, Y)$ is a bicontramodule via the structure maps

$$
\begin{aligned}
& \operatorname{Hom}_{R,-}\left(C, \operatorname{Hom}_{R,-}(C, Y)\right) \\
& \left.\quad \cong \operatorname{Hom}_{R,-}\left(C \otimes_{R} C, Y\right)\right) \stackrel{\operatorname{Hom}_{R,-}(\Delta, Y)}{\longrightarrow} \operatorname{Hom}_{R,-}(C, Y), \\
& \operatorname{Hom}_{-, R}\left(C, \operatorname{Hom}_{R,-}(C, Y)\right) \\
& \quad \cong \operatorname{Hom}_{R,-}\left(C, \operatorname{Hom}_{-, R}(C, Y)\right) \stackrel{\operatorname{Hom}_{R,-}\left(C, \beta_{r}\right)}{\longrightarrow} \operatorname{Hom}_{R,-}(C, Y) .
\end{aligned}
$$

The comonad structure is given by

$$
\operatorname{Hom}_{R,-}\left(\epsilon \otimes_{R} C, Y\right): S_{r} Y \rightarrow S_{r}^{2} Y \quad \text { and } \quad \beta_{l}: S_{r} Y \rightarrow Y .
$$

- The comonad distributive law

$$
\begin{aligned}
\Psi: \operatorname{Hom}_{-, R}\left(C, \operatorname{Hom}_{R,-}(C,-)\right) & \cong \operatorname{Hom}_{R, R}\left(C \otimes_{k} C,-\right) \\
& \cong \operatorname{Hom}_{R,-}\left(C, \operatorname{Hom}_{-, R}(C,-)\right),
\end{aligned}
$$

given by switching the arguments.

- The right $\Psi$-comodule functor $\sqcap: C$-Ctrmod- $C \rightarrow$ Mod- $k$, given by the coequalizer

$$
\operatorname{Hom}_{R, R}(C, Y) \underset{\operatorname{Hom}_{R, R}\left(R, \alpha_{r}\right)}{\stackrel{\operatorname{Hom}_{R, R}\left(R, \alpha_{l}\right)}{\longrightarrow}} \operatorname{Hom}_{R, R}(R, Y) \longrightarrow \sqcap Y .
$$

The natural transformation $i$ is given by the isomorphism

$$
i: \sqcap \operatorname{Hom}_{R,-}(C,-) \cong \operatorname{Hom}_{R, R}(R,-) \cong \sqcap \operatorname{Hom}_{-, R}(C,-) .
$$

- The left $\Psi$-comodule functor $\sqcup=\operatorname{Hom}_{R,-}(C,-)$ : Ctrmod- $B \rightarrow C$-Ctrmod- $C$. For any right $B$-contramodule $(Q, \alpha), \sqcup Q=\operatorname{Hom}_{R,-}(C, Q)$ is a $C$-bicontramodule via the structure maps

$$
\begin{aligned}
\operatorname{Hom}_{R,-}\left(C, \operatorname{Hom}_{R,-}(C, Q)\right) & \stackrel{\cong}{\longrightarrow} \operatorname{Hom}_{R,-}\left(C \otimes_{R} C, Q\right) \\
& \stackrel{\operatorname{Hom}_{R,-}(\Delta, Q)}{\longrightarrow} \operatorname{Hom}_{R,-}(C, Q), \\
\operatorname{Hom}_{-, R}\left(C, \operatorname{Hom}_{R,-}(C, Q)\right) & \stackrel{w_{Q}}{\longrightarrow} \operatorname{Hom}_{R,-}\left(C, \operatorname{Hom}_{R,-}(C, Q)\right) \\
& \left.\cong \operatorname{Hom}_{R,-}\left(C \otimes_{R} C, Q\right)\right) \\
& \stackrel{\operatorname{Hom}_{R,-}(\Delta, Q)}{\longrightarrow} \operatorname{Hom}_{R,-}(C, Q),
\end{aligned}
$$


where $w: \operatorname{Hom}_{-, R}\left(C, \operatorname{Hom}_{R,-}(C,-)\right) \rightarrow \operatorname{Hom}_{R,-}\left(C, \operatorname{Hom}_{R,-}(C,-)\right)$ is given by

$$
\left(w_{Q}(h)\right)(c)(d)=\alpha(h(d-)(c)) .
$$

For any right $B$-contramodule $(Q, \alpha)$, this determines a para-cyclic object in Mod- $k$. It is given by $\sqcap \operatorname{Hom}_{-, R}\left(C^{\otimes_{R} n+1}, \operatorname{Hom}_{R,-}(C, Q)\right) \cong \operatorname{Hom}_{R, R}\left(C^{\otimes_{R} n+1}, Q\right)$ at degree $n$. Denote $\Delta(c)=c_{\langle 1\rangle} \otimes_{R} c_{\langle 2\rangle}$. For every $0 \leq k \leq n-1$, the corresponding face map is

$$
\begin{aligned}
& \left(d_{k} \varphi^{(n)}\right)\left(c_{0} \otimes_{R} \cdots \otimes_{R} c_{n-1}\right) \\
& \quad=\varphi^{(n)}\left(c_{0} \otimes_{R} \cdots \otimes_{R} c_{k-1} \otimes_{R} \Delta\left(c_{k}\right) \otimes_{R} c_{k+1} \otimes_{R} \cdots \otimes_{R} c_{n-1}\right),
\end{aligned}
$$

while

$$
\begin{aligned}
& \left(d_{n} \varphi^{(n)}\right)\left(c_{0} \otimes_{R} \cdots \otimes_{R} c_{n-1}\right) \\
& \quad=\alpha\left(\varphi^{(n)}\left(c_{0\langle 2\rangle} \otimes_{R} c_{1} \otimes_{R} \cdots \otimes_{R} c_{n-1} \otimes_{R} c_{0\langle 1\rangle}-\right)\right) .
\end{aligned}
$$

If $0 \leq k \leq n$, then the degeneracy map $s_{k}$ is given by

$$
\begin{aligned}
& \left(s_{k} \varphi^{(n)}\right)\left(c_{0} \otimes_{R} \cdots \otimes_{R} c_{n+1}\right) \\
& \quad=\varphi^{(n)}\left(c_{0} \otimes_{R} \cdots \otimes_{R} c_{k-1} \otimes_{R} c_{k} \epsilon\left(c_{k+1}\right) \otimes_{R} c_{k+2} \otimes_{R} \cdots \otimes_{R} c_{n+1}\right) .
\end{aligned}
$$

The para-cyclic map is

$$
\left(t_{n} \varphi^{(n)}\right)\left(c_{0} \otimes_{R} \cdots \otimes_{R} c_{n}\right)=\alpha\left(\varphi^{(n)}\left(c_{1} \otimes_{R} \cdots \otimes_{R} c_{n} \otimes_{R} c_{0}-\right)\right)
$$

for $\varphi^{(n)} \in \operatorname{Hom}_{R, R}\left(C^{\otimes_{R} n+1}, Q\right)$.

This is a non-commutative base version of the para-cyclic module in [5], p. 4 (though note the minor difference of using a left or a right module coalgebra $C$ ). In [5] additional properties of the contramodule $Q$ are assumed so that the associated para-cyclic module has a cyclic subobject.

Example 5.8. Let $B$ be a right bialgebroid over a $k$-algebra $R$ and $(C, \Delta, \epsilon)$ be a left $B$-comodule coring with coaction $c \mapsto c^{[-1]} \otimes_{R} c^{[0]}$. Then $(C, \Delta, \epsilon)$ is in particular an $L:=R^{\text {op }}$-coring. An object in $\mathscr{B}$ is given by the following data:

- The same comonads $S_{l}=\operatorname{Hom}_{-, L}(C,-)$ and $S_{r}=\operatorname{Hom}_{L,-}(C,-)$ on $C$-Ctrmod- $C$ as constructed in Example 5.7 (replacing $R$ by $L$ );

- the same distributive law $\Psi$ as in Example 5.7 (replacing $R$ by $L$ );

- the same right $\Psi$-comodule functor $(\sqcap, i)$ as in Example 5.7 (replacing $R$ by $L$ );

- the left $\Psi$-comodule functor $\sqcup=\operatorname{Hom}_{L,-}(C,-): B$-Mod $\rightarrow C$-Ctrmod- $C$. For any left $B$-module $N, \sqcup N=\operatorname{Hom}_{L,-}(C, N)$ is a bicontramodule via the 
structure maps

$$
\begin{aligned}
& \operatorname{Hom}_{L,-}\left(C, \operatorname{Hom}_{L,-}(C, N)\right) \cong \operatorname{Hom}_{L,-}\left(C \otimes_{L} C, N\right) \\
& \stackrel{\operatorname{Hom}_{L,-}(\Delta, N)}{\longrightarrow} \operatorname{Hom}_{L,-}(C, N), \\
& \operatorname{Hom}_{-, L}\left(C, \operatorname{Hom}_{L,-}(C, N)\right) \stackrel{w_{N}}{\longrightarrow} \operatorname{Hom}_{L,-}\left(C, \operatorname{Hom}_{L,-}(C, N)\right) \\
& \cong \operatorname{Hom}_{L,-}\left(C \otimes_{L} C, N\right) \\
& \stackrel{\operatorname{Hom}_{L,-}(\Delta, N)}{\longrightarrow} \operatorname{Hom}_{L,-}(C, N),
\end{aligned}
$$

where $w: \operatorname{Hom}_{-, L}\left(C, \operatorname{Hom}_{L,-}(C,-)\right) \rightarrow \operatorname{Hom}_{L,-}\left(C, \operatorname{Hom}_{L,-}(C,-)\right)$ is given by

$$
\left(w_{N}(h)\right)(c)(d)=d^{[-1]} h\left(d^{[0]}\right)(c) .
$$

For any left $B$-module $N$, this determines a para-cyclic object in Mod- $k$. At degree $n$, it is given by $\sqcap \operatorname{Hom}_{-, L}\left(C^{\otimes_{L} n+1}, \operatorname{Hom}_{L,-}(C, N)\right) \cong \operatorname{Hom}_{L, L}\left(C^{\otimes_{L} n+1}, N\right)$. Denote $\Delta(c)=c_{\langle 1\rangle} \otimes_{L} c_{\langle 2\rangle}$. For every $0 \leq k \leq n-1$, the corresponding face map is

$$
\begin{aligned}
& \left(d_{k} \varphi^{(n)}\right)\left(c_{0} \otimes_{L} \cdots \otimes_{L} c_{n-1}\right) \\
& \quad=\varphi^{(n)}\left(c_{0} \otimes_{L} \cdots \otimes_{L} c_{k-1} \otimes_{L} \Delta\left(c_{k}\right) \otimes_{L} c_{k+1} \otimes_{L} \cdots \otimes_{L} c_{n-1}\right),
\end{aligned}
$$

while

$$
\begin{aligned}
& \left(d_{n} \varphi^{(n)}\right)\left(c_{0} \otimes_{L} \cdots \otimes_{L} c_{n-1}\right) \\
& \quad=c_{0\langle 1\rangle^{[-1]}} \varphi^{(n)}\left(c_{0\langle 2\rangle} \otimes_{L} c_{1} \otimes_{L} \cdots \otimes_{L} c_{n-1} \otimes_{L} c_{0\langle 1\rangle}{ }^{[0]}\right) .
\end{aligned}
$$

If $0 \leq k \leq n$, then the degeneracy map $s_{k}$ is given by

$$
\begin{aligned}
& \left(s_{k} \varphi^{(n)}\right)\left(c_{0} \otimes_{L} \cdots \otimes_{L} c_{n+1}\right) \\
& \quad=\varphi^{(n)}\left(c_{0} \otimes_{L} \cdots \otimes_{L} c_{k-1} \otimes_{L} c_{k} \epsilon\left(c_{k+1}\right) \otimes_{L} c_{k+2} \otimes_{L} \cdots \otimes_{L} c_{n+1}\right)
\end{aligned}
$$

The para-cyclic map is

$$
\left(t_{n} \varphi^{(n)}\right)\left(c_{0} \otimes_{L} \cdots \otimes_{L} c_{n}\right)=c_{0}^{[-1]} \varphi^{(n)}\left(c_{1} \otimes_{L} \cdots \otimes_{L} c_{n} \otimes_{L} c_{0}^{[0]}\right),
$$

for $\varphi^{(n)} \in \operatorname{Hom}_{L, L}\left(C^{\otimes_{L} n+1}, N\right)$.

\section{The cyclic duality functor}

The functor recently known as the cyclic duality functor, appeared first in Connes' work [8]. In its original form it is an isomorphism between the category of cyclic objects and the category of cocyclic objects in a given category. It was extended in [18] to an isomorphism between certain full subcategories of the categories of 
para-cyclic, and of para-cocyclic objects. The objects of these full subcategories are those para-(co)cyclic objects whose para-(co)cyclic morphisms are isomorphisms at all degrees. The aim of the current section is to extend cyclic duality to a functor between appropriate subcategories of $\mathcal{A}$ and $\mathcal{B}$ in Definitions 2.1 and 4.5 , respectively.

Connes's cyclic duality functor (in the extended form in [18]) and also its dual version (from a subcategory of the category of para-cyclic objects to a subcategory of the category of para-cocyclic objects) both will be denoted by (ح).

Denote by $\mathcal{A}^{\times}$the full subcategory of $\mathcal{A}$ in Definition 2.1, whose objects $\left(T_{l}, T_{r}, \Phi, \sqcap, i, \sqcup, w\right)$ obey the property that $\Phi, i$ and $w$ are natural isomorphisms. In the category $\overline{\mathcal{P}}$ in Definition 2.2, introduce the full subcategory $\overline{\mathcal{P}}^{\times}$whose objects have para-cocyclic morphisms which are natural isomorphisms at all degrees. Clearly, the functor $\mathcal{Z}^{*}$ in Theorem 2.3 induces a functor $\mathcal{Z}^{* \times}: \mathcal{A}^{\times} \rightarrow \overline{\mathcal{P}}^{\times}$. Symmetrically, introduce the full subcategory $\mathscr{B}^{\times}$of the category $\mathscr{B}$ in Definition 4.5, for whose objects $\left(S_{l}, S_{r}, \Psi, \sqcap, i, \sqcup, w\right)$ the natural transformations $\Psi, i$ and $w$ are isomorphisms. By Theorem 4.7, there is an induced functor $\mathcal{Z}_{*}^{\times}: \mathscr{B}^{\times} \rightarrow \underline{\mathcal{P}}^{\times}$, where $\underline{\mathcal{P}}^{\times}$is the full subcategory of $\mathcal{P}$, for whose objects the para-cyclic morphisms are natural isomorphisms at all degrees. Finally, denote by $\mathcal{A}_{c}^{\times}$the full subcategory of $\mathcal{A}^{\times}$, for whose objects $\left(T_{l}, T_{r}, \Phi, \sqcap, i, \sqcup, w\right)$ the codomain category $\mathcal{C}$ of the right $\Phi$-module functor $\Pi$ possesses coequalizers. Symmetrically, denote by $\mathscr{B}_{e}^{\times}$the full subcategory of $\mathscr{B}^{\times}$, for whose objects $\left(S_{l}, S_{r}, \Psi, \sqcap, i, \sqcup, w\right)$ the codomain category $\mathcal{C}$ of the right $\Psi$-comodule functor $\Pi$ possesses equalizers. Restrictions of the functor $\mathcal{Z}^{* \times}$ to $\mathcal{A}_{c}^{\times}$and $\mathcal{Z}_{*}^{\times}$to $\mathscr{B}_{e}^{\times}$are denoted by the same symbols $\mathcal{Z}^{* \times}$ and $\mathcal{Z}_{*}^{\times}$.

Theorem 6.1. Using the notation in the paragraph preceding the theorem, there exists a functor $(\triangle): \mathcal{A}_{c}^{\times} \rightarrow \mathscr{B}^{\times}$such that the diagram

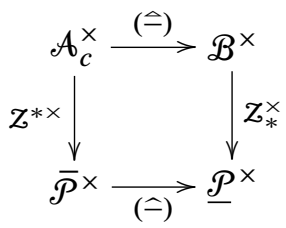

commutes up to a natural isomorphism.

Symmetrically, there exists a functor $(\triangle): \mathscr{B}_{e}^{\times} \rightarrow \mathcal{A}^{\times}$such that the functors $\mathfrak{Z}^{* \times}(\triangleq)$ and $\overline{Z_{*}^{\times}(-)}$are naturally isomorphic.

The proof of Theorem 6.1 goes through a series of lemmata.

Recall that for the Eilenberg-Moore category $\mathcal{M}^{T}$ of a monad $T: \mathcal{M} \rightarrow \mathcal{M}$, there is a forgetful functor $U: \mathcal{M}^{T} \rightarrow \mathcal{M}$, with object map $(M, \varrho) \mapsto M$ and acting on the morphisms as the identity map. The forgetful functor $U$ has a left adjoint $F$, with object map $M \mapsto(T M, m M)$ and morphism map $f \mapsto T f$. 
Lemma 6.2. Let $\left(T_{l}, m_{l}, u_{l}\right)$ and $\left(T_{r}, m_{r}, u_{r}\right)$ be monads on the same category $\mathcal{M}$ and $\Phi: T_{r} T_{l} \rightarrow T_{l} T_{r}$ be a distributive law which is a natural isomorphism. Consider the induced monad (1.1) and the forgetful functor $U: \mathcal{M}^{T_{l} T_{r}} \rightarrow \mathcal{M}$.

(1) There is a comonad $\left(\hat{T}_{l}, d_{l}, e_{l}\right)$ on $\mathcal{M}^{T_{l} T_{r}}$ such that $U \hat{T}_{l}=T_{l} U, U d_{l}=$ $T_{l} u_{l} U$ and $U e_{l}(M, \varrho)=\varrho \circ T_{l} u_{r} M$ for any $T_{l} T_{r}$-algebra $(M, \varrho)$.

(2) There is a comonad $\left(\hat{T}_{r}, d_{r}, e_{r}\right)$ on $\mathcal{M}^{T_{l} T_{r}}$ such that $U \hat{T}_{r}=T_{r} U, U d_{r}=$ $T_{r} u_{r} U$ and $U e_{r}(M, \varrho)=\varrho \circ u_{l} T_{r} M$ for any $T_{l} T_{r}$-algebra $(M, \varrho)$.

(3) There is a comonad distributive law $\widehat{\Phi}: \widehat{T}_{l} \widehat{T}_{r} \rightarrow \widehat{T}_{r} \widehat{T}_{l}$ such that $U \widehat{\Phi}=\Phi^{-1} U$.

Proof. (1) By Beck's classical theorem [1], p. 122, the distributive law $\Phi$ induces a monad $\left(\widetilde{T}_{l}, \tilde{m}_{l}, \tilde{u}_{l}\right)$ on the category of $T_{r}$-algebras such that the forgetful functor $U_{r}: \mathcal{M}^{T_{r}} \rightarrow \mathcal{M}$ satisfies

$$
U_{r} \widetilde{T}_{l}=T_{l} U_{r}, \quad U_{r} \tilde{m}_{l}=m_{l} U_{r}, \quad U_{r} \tilde{u}_{l}=u_{l} U_{r} .
$$

Moreover, the category $\left(\mathcal{M}^{T_{r}}\right)^{\widetilde{T}_{l}}$ of $\widetilde{T}_{l}$-algebras is isomorphic to the category of $T_{l} T_{r^{-}}$ algebras. Consider the forgetful functor $\widetilde{U}_{l}:\left(\mathcal{M}^{T_{r}}\right)^{\tilde{T}_{l}} \rightarrow \mathcal{M}^{T_{r}}$ and its left adjoint $\widetilde{F}_{l}$. The composite functor $\widetilde{U}_{l} \widetilde{F}_{l}$ is equal to $\widetilde{T}_{l}$ while $\widehat{T}_{l}:=\widetilde{F}_{l} \widetilde{U}_{l}$ is a comonad on $\left(\mathcal{M}^{T_{r}}\right)^{\tilde{T}_{l}} \cong \mathcal{M}^{T_{l} T_{r}}$. Its comultiplication is given by $\widetilde{F}_{l} \tilde{u}_{l} \widetilde{U}_{l}: \widetilde{F}_{l} \widetilde{U}_{l} \rightarrow \widetilde{F}_{l} \widetilde{T}_{l} \widetilde{U}_{l}=$ $\widetilde{F}_{l} \widetilde{U}_{l} \widetilde{F}_{l} \widetilde{U}_{l}$ and the counit is $\tilde{\varrho}_{l}: \widetilde{F}_{l} \widetilde{U}_{l}\left(M, \tilde{\varrho}_{l}\right)=\left(\widetilde{T}_{l} M, \tilde{m}_{l} M\right) \rightarrow\left(M, \tilde{\varrho}_{l}\right)$ for any $\widetilde{T}_{l}$-algebra $\left(M, \tilde{\varrho}_{l}\right)$. Since the composite functor $U_{r} \widetilde{U}_{l}$ differs from the forgetful functor $U: \mathcal{M}^{T_{l} T_{r}} \rightarrow \mathcal{M}$ by the isomorphism $\left(\mathcal{M}^{T_{r}}\right)^{\widetilde{T}_{l}} \cong \mathcal{M}^{T_{l} T_{r}}$, the comonad $\hat{T}_{l}$ obeys the required properties.

Part (2) follows by applying the same reasoning as in part (1) to the distributive law $\Phi^{-1}$.

For any $T_{l} T_{r}$-algebra $(M, \varrho)$, the $T_{l} T_{r}$-actions on $\widehat{T}_{l}(M, \varrho)$ and $\widehat{T}_{r}(M, \varrho)$ are given by the respective morphisms $\hat{\rho}_{l}: T_{l} T_{r} T_{l} M \rightarrow T_{l} M$ and $\hat{\rho}_{r}: T_{l} T_{r} T_{r} M \rightarrow T_{r} M$, where

$$
\begin{aligned}
& \hat{\rho}_{l}:=T_{l} \varrho \circ T_{l} u_{l} T_{r} M \circ m_{l} T_{r} M \circ T_{l} \Phi M, \\
& \hat{\rho}_{r}:=T_{r} \varrho \circ T_{r} T_{l} u_{r} M \circ \Phi^{-1} M \circ T_{l} m_{r} M .
\end{aligned}
$$

(3) The composite monad $T_{l} T_{r}$ in (1.1) induces a monad Cat $\left(\mathcal{M}, T_{l} T_{r}\right)$ on the category $\operatorname{Cat}(\mathcal{M}, \mathcal{M})$ of functors $\mathcal{M} \rightarrow \mathcal{M}$. The natural transformation $\Phi^{-1}$ yields a morphism of its algebras $\left(T_{l} T_{r}, T_{l} m_{r} \circ m_{l} T_{r} T_{r} \circ T_{l} \Phi T_{r}\right) \rightarrow\left(T_{r} T_{l}, T_{r} m_{l} \circ \Phi^{-1} T_{l} \circ\right.$ $\left.T_{l} m_{r} T_{l}\right)$. Indeed, using string computation, we have

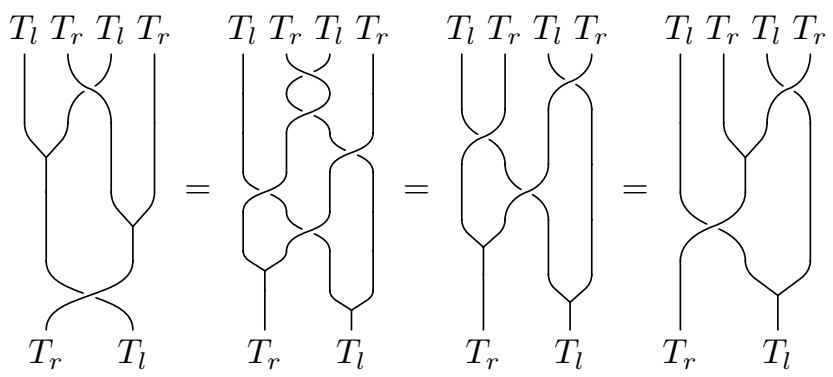


where for the first and the third equations one uses the definition of distributive laws and the second relation follows by $\Phi^{-1} \circ \Phi=T_{r} T_{l}$. Comparing these actions with (6.1) and (6.2), this implies the existence of a natural transformation $\widehat{\Phi}: \widehat{T}_{l} \hat{T}_{r} \rightarrow \widehat{T}_{r} \widehat{T}_{l}$ such that $U \widehat{\Phi}=\Phi^{-1} U$. Since the forgetful functor $U$ reflects isomorphisms, $\widehat{\Phi}$ is a natural isomorphism. Using that $U$ is faithful, it is easy to check that since $\Phi$ is a monad distributive law, $\widehat{\Phi}$ is a comonad distributive law.

For an object $\left(T_{l}, T_{r}, \Phi, \sqcap: \mathcal{M} \rightarrow \mathcal{C}, i, \sqcup: \mathscr{D} \rightarrow \mathcal{M}, w\right)$ of $\mathcal{A}_{c}^{\times}$, consider the forgetful functor $U: \mathcal{M}^{T_{l} T_{r}} \rightarrow \mathcal{M}$ and the natural transformations $\xi_{l}: T_{l} U \rightarrow U$ and $\xi_{r}: T_{r} U \rightarrow U$, given for any $T_{l} T_{r}$-algebra $(M, \varrho)$ by the morphisms

$$
\xi_{l}(M, \varrho):=\varrho \circ T_{l} u_{r} M \quad \text { and } \quad \xi_{r}(M, \varrho):=\varrho \circ u_{l} T_{r} M .
$$

Since coequalizers in $\mathcal{\ell}$ exist by assumption, we can define a functor $\bar{\Pi}: \mathcal{M}^{T_{l} T_{r}} \rightarrow \mathcal{C}$ via the coequalizer

$$
\sqcap T_{l} U \underset{\square \xi_{r} \circ i U}{\stackrel{\Pi \xi_{l}}{\underset{n}{m}}} \sqcap U \stackrel{p}{\rightarrow} \hat{\sqcap}
$$

in the category of functors. For any $T_{l} T_{r}$-algebra $(M, \varrho), p(M, \varrho): \sqcap M \rightarrow \hat{\Pi}(M, \varrho)$ is the coequalizer of $\sqcap \varrho \circ \sqcap T_{l} u_{r} M$ and $\sqcap \varrho \circ \sqcap u_{l} T_{r} M \circ i M$. For any morphism $f:(M, \varrho) \rightarrow\left(M^{\prime}, \varrho^{\prime}\right)$ in $\mathcal{M}^{T_{l} T_{r}}$, the composite $p\left(M^{\prime}, \varrho^{\prime}\right) \circ \sqcap f$ coequalizes the parallel morphisms in (6.4) (evaluated at $(M, \varrho)$ ). Hence we can define $\bar{\Pi} f$ as the unique morphism for which $\hat{\Pi} f \circ p(M, \varrho)=p\left(M^{\prime}, \varrho^{\prime}\right) \circ \sqcap f$.

Lemma 6.3. Consider an object $\left(T_{l}, T_{r}, \Phi, \sqcap, i, \sqcup, w\right)$ of $\mathcal{A}_{c}^{\times}$. For the forgetful functor $U: \mathcal{M}^{T_{l} T_{r}} \rightarrow \mathcal{M}$, the monads $\widehat{T}_{l}$ and $\hat{T}_{r}$ in Lemma 6.2 and the functor $\hat{\Pi}$ in (6.4), there are natural isomorphisms $\theta_{l}: \widehat{\Pi}_{T_{l}} \rightarrow \sqcap U$ and $\theta_{r}: \hat{\Pi} \widehat{T}_{r} \rightarrow \sqcap U$ such that

$$
\theta_{l} \circ p \widehat{T}_{l} \circ i^{-1} U=\sqcap \xi_{r} \text { and } \theta_{r} \circ p \widehat{T}_{r} \circ i U=\sqcap \xi_{l} .
$$

Proof. By definition, $\hat{\Pi}_{T_{r}}$ is the coequalizer of the natural transformations $\sqcap T_{r} \xi_{l}$ 。 $\sqcap \Phi^{-1} U$ and $\sqcap m_{r} U \circ i T_{r} U$. Since $\Phi$ is an isomorphism, this is equivalent to the coequalizer of $\sqcap T_{r} \xi_{l}$ and $\sqcap m_{r} U \circ i T_{r} U \circ \sqcap \Phi U=i U \circ \sqcap m_{l} U \circ i^{-1} T_{l} U$, where we used that $(\sqcap, i)$ is a right $\Phi$-module functor, cf. first condition in (1.3). Using that $i$ is an isomorphism, we conclude that $\bar{\Gamma} \widehat{T}_{r}$ is the coequalizer of $i^{-1} U \circ \Pi T_{r} \xi_{l} \circ i T_{l} U=$ $\sqcap T_{l} \xi_{l}$ and $\sqcap m_{l} U$. The coequalizer

$$
T_{l} T_{l} U \underset{m_{l} U}{\stackrel{T_{l} \xi_{l}}{\longrightarrow}} T_{l} U \stackrel{\xi_{l}}{\longrightarrow} U
$$

is split, as $\left(M, \xi_{l}(M, \varrho)\right)$ is a $T_{l}$-algebra for any $T_{l} T_{r}$-module $(M, \varrho)$. Hence it is preserved by composing with $\square$ on the left. Thus, by the universal property of coequalizers, there is a unique natural isomorphism $\theta_{r}: \vec{\Pi}_{r} \rightarrow \sqcap U$ such that $\theta_{r}$ 。 $p \widehat{T}_{r} \circ i U=\sqcap \xi_{l}$. The existence of the isomorphism $\theta_{l}: \widehat{\Pi}_{T_{l}} \rightarrow \sqcap U$ is proven similarly. 
Lemma 6.4. Consider an object $\left(T_{l}, T_{r}, \Phi, \sqcap, i, \sqcup, w\right)$ of $\mathcal{A}_{c}^{\times}$. Then $T_{r} \sqcup$ is a left module functor for the composite monad $T_{l} T_{r}$ in (1.1). Hence there is a functor பे: $\mathscr{D} \rightarrow \mathcal{M}^{T_{l} T_{r}}$, with object map and morphism map

$$
\text { பิ } Y:=\left(T_{r} \sqcup Y, w^{-1} Y \circ m_{l} \sqcup Y \circ T_{l} w Y \circ T_{l} m_{r} \sqcup Y\right) \quad \text { and } \quad \hat{\sqcup} f:=T_{r} \sqcup f \text {. }
$$

Proof. Unitality of the given $T_{l} T_{r}$-action is immediate by unitality of the multiplications of $T_{l}$ and $T_{r}$. Associativity is checked as follows.

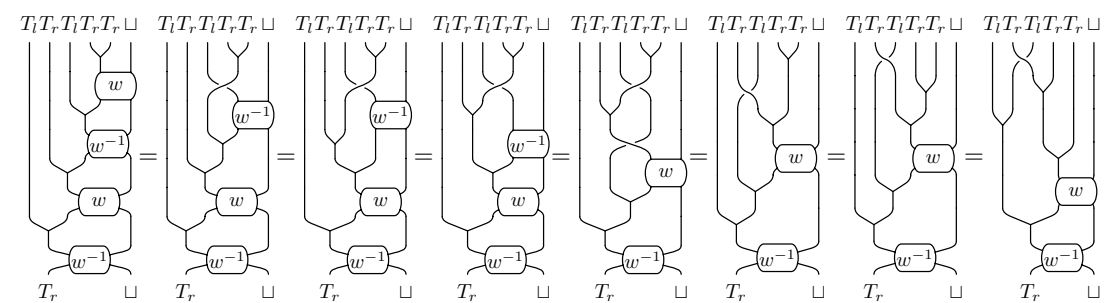

The first and the fourth equalities follow by using that $(\sqcup, w)$ is a left $\Phi$-module functor, cf. first identity in (1.4). The second equality follows by associativity of $m_{r}$. Naturality is used in the third equality, and also in the last two ones, in the case of the last equality together with the associativity of $m_{l}$ and of $m_{r}$. The fifth equality is a consequence of the fact that $\Phi$ is a distributive law.

Proof of Theorem 6.1. Let $\left(T_{l}, T_{r}, \Phi, \sqcap, i, \sqcup, w\right)$ be an object in $\mathcal{A}_{c}^{\times}$, with $\sqcap: \mathcal{M} \rightarrow$ $\mathcal{C}$ and $\sqcup: \mathscr{D} \rightarrow \mathcal{M}$. First we show that the septuple $\left(\widehat{T}_{l}, \widehat{T}_{r}, \widehat{\Phi}, \hat{\Pi}, \hat{i}, \hat{\sqcup}, \hat{w}\right)$ is an object of $\mathcal{B}$, where $U: \mathcal{M}^{T_{l} T_{r}} \rightarrow \mathcal{M}$ is the forgetful functor, the comonads $\widehat{T}_{l}$ and $\widehat{T}_{r}$ and the comonad distributive law $\widehat{\Phi}$ are constructed as in Lemma 6.2 , the functor $\hat{\Pi}$ is defined by (6.4), the functor $\vec{\sqcup}$ is constructed as in Lemma 6.4, while $\hat{i}$ and $\hat{w}$ are given by the relations

$$
\hat{i}:=\theta_{l}^{-1} \circ \theta_{r} \quad \text { and } \quad U \hat{w}:=T_{r} w^{-1} \circ \Phi^{-1} \sqcup .
$$

The following string computation proves that $\hat{w} Y: \widehat{T}_{l} \hat{\mathrm{\Delta} Y} \rightarrow \hat{T}_{r} \hat{\mathrm{U} Y}$ is a morphism in $\mathcal{M}^{T_{l} T_{r}}$ for any object $Y$ in $\mathcal{D}$.

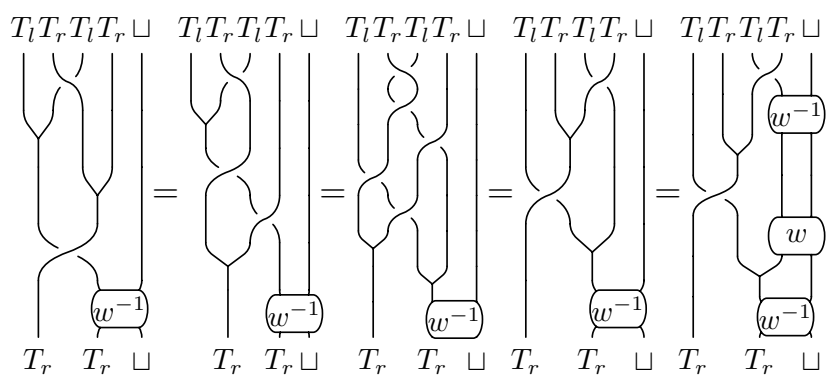

Here we used the form of the $T_{l} T_{r}$-actions on $\hat{T}_{l}(M, \varrho), \hat{T}_{r}(M, \varrho)$ and $\sqcup \hat{Y} Y$, for any $T_{l} T_{r}$-algebra $(M, \varrho)$ and for any object $Y$ of $\mathscr{D}$; see relations (6.1), (6.2) and 
Lemma 6.4. For the first, second and third equalities we also used that $\Phi$ is a distributive law and that the multiplication of a monad is a natural transformation. Hence $\hat{w}$ can be regarded as a natural transformation $\widehat{T}_{l} \overrightarrow{ } \rightarrow \widehat{T}_{r} \Delta$.

By naturality of $p$ in (6.4) and by Lemma 6.2,

$$
\begin{aligned}
& p \hat{T}_{r}^{2} \circ \sqcap T_{r} u_{r} U=\hat{\Pi}_{r} \circ p \hat{T}_{r}, \\
& p \widehat{T}_{l}^{2} \circ \sqcap T_{l} u_{l} U=\hat{\Pi} d_{l} \circ p \hat{T}_{l}, \\
& p \hat{T}_{r} \widehat{T}_{l} \circ \sqcap \Phi^{-1} U=\hat{\Pi} \widehat{\Phi} \circ p \hat{T}_{l} \hat{T}_{r} .
\end{aligned}
$$

Using Lemma 6.3 (in the first and third equalities), the second relation in (1.3) on $i$ and unitality of $\xi_{r}$ (in the second equality) and the first equality in (6.5) defining $\hat{i}$ (in the fourth equality), we see that

$$
\begin{aligned}
p \hat{T}_{l} \circ \sqcap u_{l} U \circ \sqcap \xi_{l} \circ i^{-1} U & =\theta_{l}^{-1} \circ \sqcap \xi_{r} \circ i U \circ \sqcap u_{l} U \circ \sqcap \xi_{l} \circ i^{-1} U \\
& =\theta_{l}^{-1} \circ \sqcap \xi_{l} \circ i^{-1} U \\
& =\theta_{l}^{-1} \circ \theta_{r} \circ p \hat{T}_{r} \\
& =\hat{i} \circ p \hat{T}_{r} .
\end{aligned}
$$

The first condition in (4.1) for $(\hat{\Pi}, \hat{i})$ can be expressed as commutativity of the inner square in the diagram below. Since $p \widehat{T}_{r}$ is a natural epimorphism, the above considerations imply that it holds true if and only if the outer square in

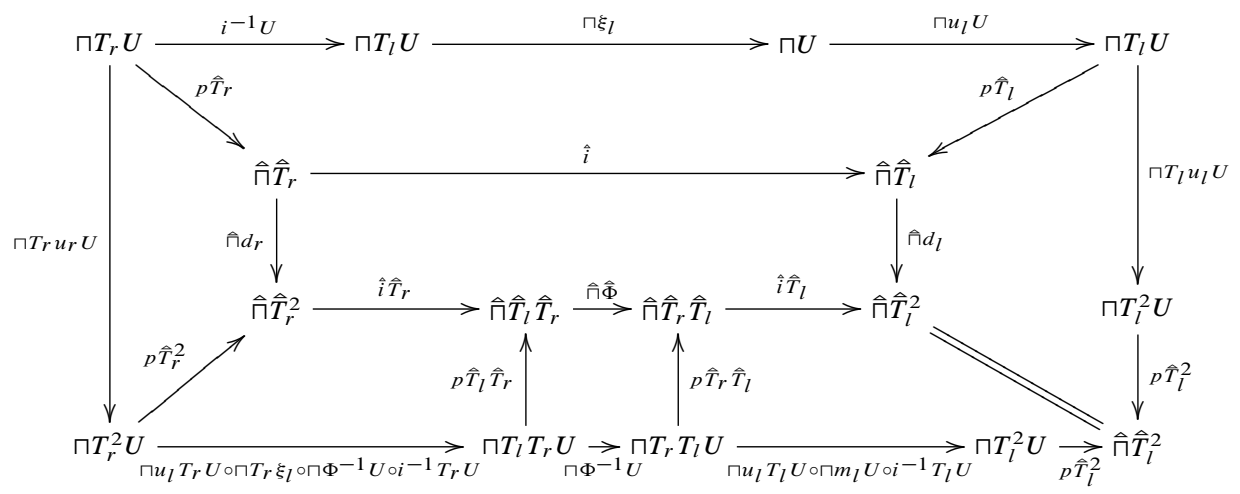

commutes, which follows from the compatibility of $\Phi$ and $i$ with the units $u_{l}$ and $u_{r}$ of both monads and unitality of the multiplication $m_{l}$; cf. the following string computation. 


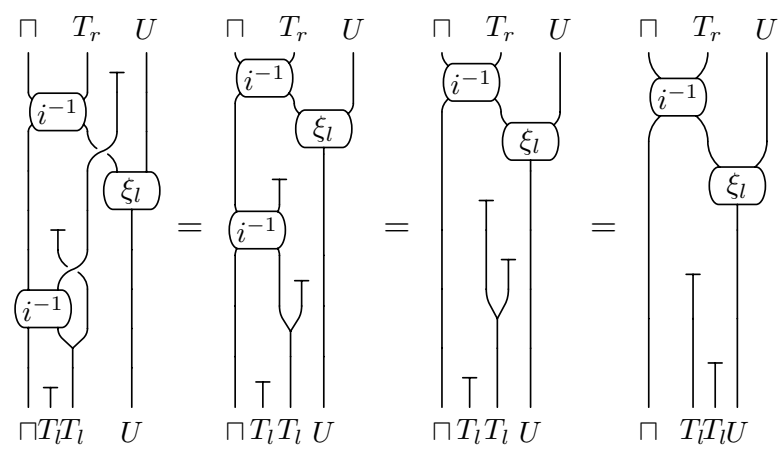

Similarly, by (6.6), by naturality of $p$ and Lemma 6.2, the second condition in (4.1) for $(\hat{\Pi}, \hat{i})$, i.e., the identity $\vec{\Pi} e_{l} \circ \hat{i}=\vec{\Pi} e_{r}$, holds true if and only of the outer square in

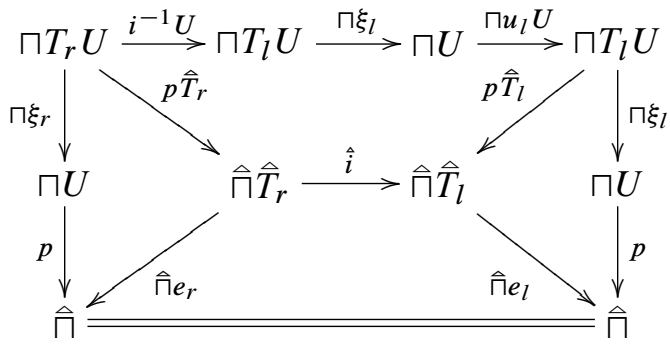

commutes, which follows by the unitality of $\xi_{l}$ and the definition of $p$ via the coequalizer in (6.4).

Next we prove that $(\vec{\sqcup}, \vec{w})$ satisfies the conditions in (4.1). Since $U$ is faithful, applying it to the first relation in (4.1) we obtain an equivalent condition. In view of Lemma 6.2 and the construction of $\hat{w}$ via the second equality in (6.5), it takes the form

$$
\begin{gathered}
T_{r}^{2} w^{-1} \circ T_{r} \Phi^{-1} \sqcup \circ \Phi^{-1} T_{r} \sqcup \circ T_{l} T_{r} w^{-1} \circ T_{l} \Phi^{-1} \sqcup \circ T_{l} u_{l} T_{r} \sqcup \\
=T_{r} u_{r} T_{r} \sqcup \circ T_{r} w^{-1} \circ \Phi^{-1} \sqcup
\end{gathered}
$$

This holds true by the computation below, where we use the compatibility between the unit of $T_{l}$ with $\Phi$ and $w$, and the fact that $u_{l}$ is a natural transformation.

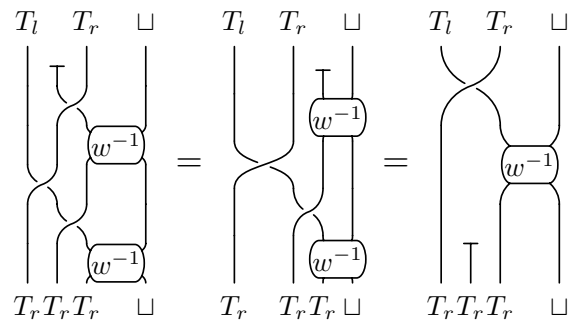


The second relation in (4.1) may be proved analogously, showing that it is equivalent to the fact that $w$ is compatible with the multiplications of $T_{l}$ and $T_{r}$. Consequently $\left(\widehat{T}_{l}, \widehat{T}_{r}, \widehat{\Phi}, \hat{\Pi}, \hat{i}, \hat{w}\right)$ is an object of $\mathcal{B}$.

Next we define the functor $(\triangle)$ on morphisms. Let $\left(G, q_{l}, q_{r}, \wedge, \pi, \vee, \omega\right)$ be a morphism in $\mathcal{A}_{c}^{\times}$. Its image in $\mathcal{B}^{\times}$under $(\triangleq)$ will be denoted by $\left(\widehat{G}, \widehat{q}_{l}, \widehat{q}_{r}, \wedge, \vec{\pi}, \vee, \widehat{\omega}\right)$. The functor $\widehat{G}: \mathcal{M}^{\prime T_{l}^{\prime} T_{r}^{\prime}} \rightarrow \mathcal{M}^{T_{l} T_{r}}$ is defined, for $(M, \rho)$ in $\mathcal{M}^{\prime} T_{l}^{\prime} T_{r}^{\prime}$, by $\widehat{G}(M, \rho)=$ $(G M, \hat{\rho})$, where

$$
\hat{\rho}=G \rho \circ q_{l} T_{r}^{\prime} M \circ T_{l} q_{r} M .
$$

On a morphism $f$ in $\mathcal{M}^{\prime} T_{l}^{\prime} T_{r}^{\prime}$, we put $\widehat{G} f=G f$. In fact, $\widehat{G}$ is the lifting of $G$ (in the sense that $U \widehat{G}=G U^{\prime}$ ), which is induced by the monad morphism $q_{l} T_{r}^{\prime} \circ T_{l} q_{r}$, cf. Lemma 1.4 and [14], Lemma 1. Since

$$
\begin{aligned}
& \widehat{T}_{l} \widehat{G}(M, \rho) \\
& =\left(T_{l} G M, T_{l} G \rho \circ T_{l} q_{l} T_{r}^{\prime} M \circ T_{l}^{2} q_{r} M \circ T_{l} u_{l} T_{r} G M \circ m_{l} T_{r} G M \circ T_{l} \Phi G M\right), \\
& \widehat{G} \widehat{T}_{l}^{\prime}(M, \rho) \\
& =\left(G T_{l}^{\prime} M, G T_{l}^{\prime} \rho \circ G T_{l}^{\prime} u_{l}^{\prime} T_{r}^{\prime} M \circ G m_{l}^{\prime} T_{r}^{\prime} M \circ G T_{l}^{\prime} \Phi^{\prime} M \circ q_{l} T_{r}^{\prime} T_{l}^{\prime} M \circ T_{l} q_{r} T_{l}^{\prime} M\right),
\end{aligned}
$$

we can check easily that $q_{l} M: T_{l} G M \rightarrow G T_{l}^{\prime} M$ is a morphism of $T_{l} T_{r}$-algebras with respect to the above actions. Hence one may define $\hat{q}_{l}: \hat{T}_{l} \widehat{G} \rightarrow \vec{G} \hat{T}_{l}^{\prime}$ by $U \hat{q}_{l}(M, \rho):=q_{l} M$ and, proceeding similarly, one may take $\hat{q}_{r}: \widehat{T}_{r} \widehat{G} \rightarrow \widehat{G} \hat{T}_{r}^{\prime}$ to be defined by $U \hat{q}_{r}(M, \rho):=q_{r} M$.

The left square in the following diagram is commutative by naturality, if choosing the upper ones of the parallel arrows. It is commutative also choosing the lower ones of the parallel arrows, by (1.5) and naturality. Therefore, universality of the coequalizer in the top row implies the existence of a unique natural transformation $\hat{\pi}$, rendering commutative the diagram.

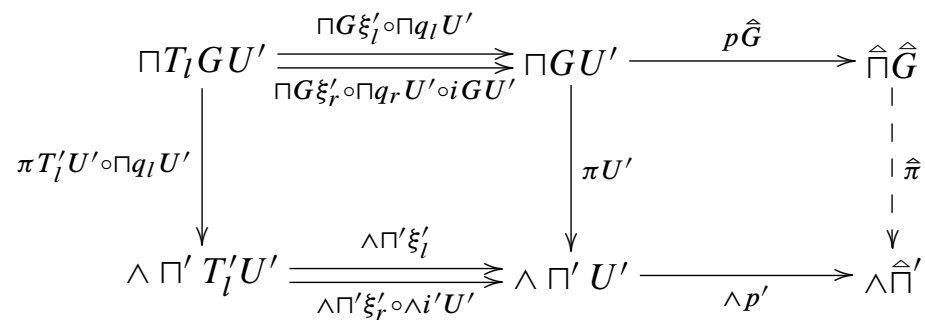

Finally, we put $U \hat{\omega}:=q_{r} \sqcup^{\prime} \circ T_{r} \omega$. We need to show that this defines indeed a natural transformation $\hat{\omega}: \hat{\sqcup} \vee \rightarrow \widehat{G} \sqcup^{\prime}$; that is, that $q_{r} \sqcup^{\prime} \circ T_{r} \omega$ commutes with the $T_{l} T_{r}$-actions on $U \hat{\sqcup} \vee=T_{r} \sqcup \vee$ and $U \hat{G} \vec{\cup}^{\prime}=G T_{r}^{\prime} \sqcup^{\prime}$. For a diagrammatic proof see the following computation, where for the first and third equations one uses that $\left(G, q_{l}\right)$ and $\left(G, q_{r}\right)$ are morphisms of monads. The second and the fourth relations 
follow by (1.6). In the first three equalities we also use that all maps are natural transformations.
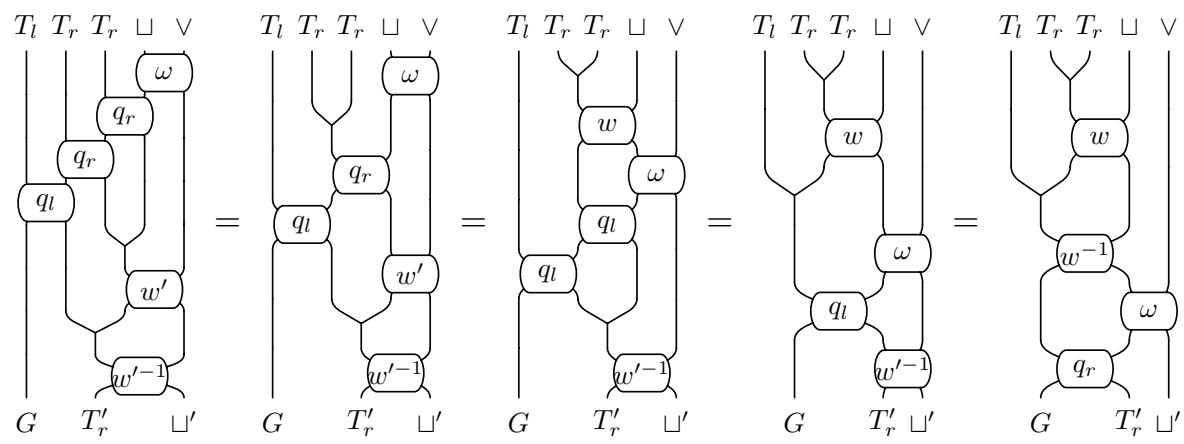

We turn to proving that the so constructed datum $\left(\widehat{G}, \hat{q}_{l}, \hat{q}_{r}, \wedge, \vec{\pi}, \vee, \widehat{\omega}\right)$ yields a morphism in $\mathcal{B}$. In order to check that $\left(G, \hat{q}_{r}\right)$ is a comonad morphism, it suffices to show that

$$
U \hat{q}_{r} \hat{T}_{r}^{\prime} \circ U \hat{T}_{r} \hat{q}_{r} \circ U d_{r} \widehat{G}=U \widehat{G} d_{r}^{\prime} \circ U \hat{q}_{r}
$$

and

$$
U e_{r} \widehat{G}=U \widehat{G} e_{r}^{\prime} \circ U \hat{q}_{r}
$$

since the forgetful functor $U$ is faithful. By Lemma 6.2, the first condition is equivalent to

$$
q_{r} T_{r}^{\prime} U^{\prime} \circ T_{r} q_{r} U^{\prime} \circ T_{r} u_{r} G U^{\prime}=G T_{r}^{\prime} u_{r}^{\prime} U^{\prime} \circ q_{r} U^{\prime},
$$

which holds true since $\left(G, q_{r}\right)$ is a monad morphism and by naturality. The second condition holds true by construction of the functor $\hat{G}$ (cf. (6.7)) and the relations in Lemma 6.2 on $e_{r}$ and $e_{r}^{\prime}$. Symmetrically, $\left(G, \hat{q}_{l}\right)$ is a comonad morphism as well. By faithfulness of $U$, the first condition in (4.2) is equivalent to

$$
q_{r} T_{l}^{\prime} U^{\prime} \circ T_{r} q_{l} U^{\prime} \circ \Phi^{-1} G U^{\prime}=G \Phi^{\prime-1} U^{\prime} \circ q_{l} T_{r}^{\prime} U^{\prime} \circ T_{l} q_{r} U^{\prime},
$$

which holds true by (1.2). The second condition in (4.2) is equivalent to commutativity of the inner square in the following diagram. Since $p \hat{T}_{r} \widehat{G}$ is a natural epimorphism, it follows by the constructions of the morphisms $\hat{q}_{r}, \hat{q}_{l}$ and $\hat{\pi}$ any by the equality 
(6.6) that the second condition in (4.2) holds true if and only if the outer square in

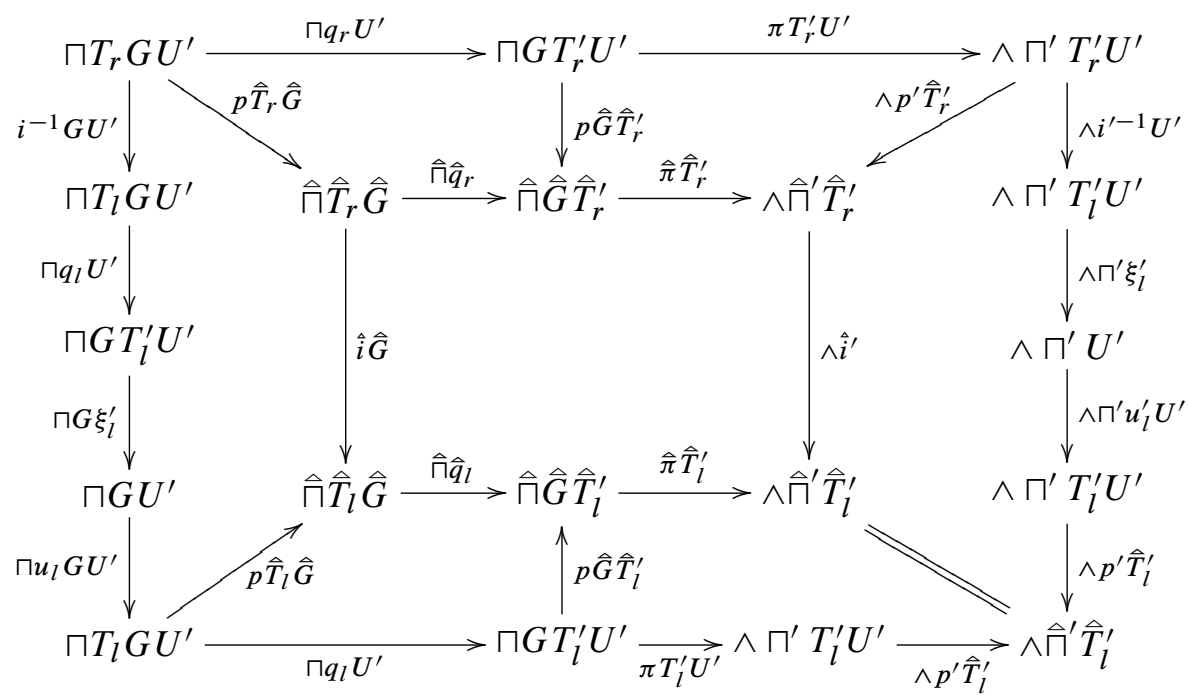

commutes. This follows by the compatibility of $q_{l}$ with $u_{l}$, naturality and (1.5). Finally, by faithfulness of $U,(4.3)$ is equivalent to

$$
\begin{aligned}
& G T_{r}^{\prime} w^{\prime-1} \circ G \Phi^{\prime-1} \sqcup^{\prime} \circ q_{l} T_{r}^{\prime} \sqcup^{\prime} \circ T_{l} q_{r} \sqcup^{\prime} \circ T_{l} T_{r} \omega \\
& \quad=q_{r} T_{r}^{\prime} \sqcup^{\prime} \circ T_{r} q_{r} \sqcup^{\prime} \circ T_{r}^{2} \omega \circ T_{r} w^{-1} \vee \circ \Phi^{-1} \sqcup \vee .
\end{aligned}
$$

This holds true by naturality, (1.2) and (1.6). This finishes the construction of the functor $(\Delta)$. It is straightforward to see that it is a functor indeed, i.e., it preserves identity morphisms and composition.

It remains to construct a natural isomorphism $\tau: Z_{*}^{\times}(\triangleq) \rightarrow \widehat{Z^{* \times}(-)}$. For a given

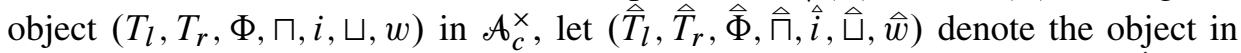
$\mathfrak{B}^{\times}$, constructed as in the first part of the proof. The para-cyclic object $\hat{\mathcal{Z}}_{*}^{\times}:=$ $\mathcal{Z}_{*}^{\times}\left(\hat{T}_{l}, \hat{T}_{r}, \widehat{\Phi}, \hat{\Pi}, \hat{i}, \vec{\sqcup}, \hat{w}\right)$ associated to the latter object as in Theorem 4.7 is given, at any non-negative degree $n$, by the functor $\hat{\Pi}_{T_{l}}^{n+1} \cup$. The para-cocyclic object $\mathfrak{Z}^{* \times}:=Z^{* \times}\left(T_{l}, T_{r}, \Phi, \sqcap, i, \sqcup, w\right)$ in Theorem 2.3 (and thus also its cyclic dual) is given at degree $n$ by $\sqcap T_{l}^{n+1} \sqcup$. The desired natural transformation $\tau_{n}$ is defined as the composition of the two morphisms

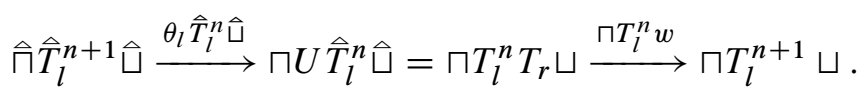

Clearly $\tau_{n}$ is a natural isomorphism. We claim that $\tau_{*}$ is also an isomorphism of para-cyclic objects between $\hat{\mathfrak{Z}}_{*}^{\times}$and the cyclic dual $\widehat{Z^{* x}}$ of $Z^{* x}$. Applying Connes's cyclic duality functor (in the form it can be found in [18]) to $Z^{* \times}$, for every degree $n$ the para-cyclic morphism $\hat{t}_{n}: \sqcap T_{l}^{n+1} \sqcup \rightarrow \sqcap T_{l}^{n+1} \sqcup$ of $\widehat{\mathcal{Z}^{* \times}}$ comes out as

$$
\hat{t}_{n}=i^{-1} T_{l}^{n} \sqcup \circ \sqcap \Phi^{-1} T_{l}^{n-1} \sqcup \circ \cdots \circ \sqcap T_{l}^{n-1} \Phi^{-1} \sqcup \circ \sqcap T_{l}^{n} w^{-1} .
$$


On the other hand, by Theorem 4.7, the para-cyclic morphism $\hat{t}_{n}: \hat{\Pi}_{l}^{n+1} \hat{\mathrm{U}} \rightarrow$ $\hat{\Pi} \hat{T}_{l}^{n+1} \cup \hat{\mathrm{of}} \hat{\mathcal{Z}}_{*}^{\times}$is given by

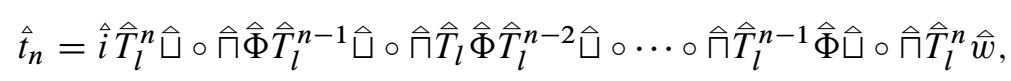

for every $n$. Since $p \widehat{T}^{n+1} \hat{\mathrm{U}}$ is an epimorphism, $\tau_{n}$ commutes with these para-cyclic morphisms if and only if

$$
\hat{t}_{n} \circ \tau_{n} \circ p \widehat{T}^{n+1} \hat{\sqcup}=\tau_{n} \circ \hat{t}_{n} \circ p \widehat{T}^{n+1} \hat{\mathrm{U}} .
$$

For the proof of this relation, using string computations, see the following sequence of equations.
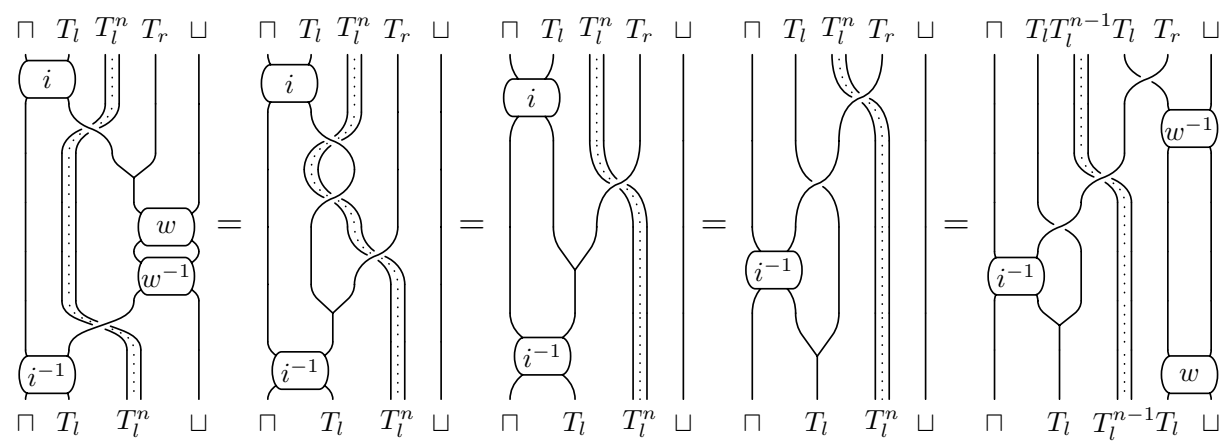

Taking into account the formulae that give $\hat{t}_{n}$ and $\tau_{n}$, on one hand, and the defining relation of $\theta_{l}$ in Lemma 6.3 together with the definition of $\xi_{r}$ in (6.3), on the other hand, one can see that the first diagram represents the left-hand side of (6.9). In view of the definition of the maps $\hat{t}_{n}, \hat{i}_{n}, \hat{w}_{n}, \widehat{\Phi}$ and $\theta_{r}$, the last diagram corresponds to the right-hand side of (6.9). To deduce the first equality we use $n$ times the compatibility relation between the multiplication of $T_{r}$ and $\Phi$. The second relation is a consequence of the fact that the string and the stripe may be unknotted, by using repeatedly the relation $\Phi^{-1} \circ \Phi=T_{r} T_{l}$. For the third equation one uses that $(\sqcap, i)$ is right $\Phi$-module functor, i.e., the first condition in (1.3). The last relation follows by using that $w$ is natural.

For $0 \leq k \leq n$, let $\hat{d}_{k}:=\hat{\Pi} \hat{T}_{l}^{k} e_{l} \widehat{T}_{l}^{n-k} \hat{\sqcup}$ be the face maps of $\hat{\mathcal{Z}}_{*}^{\times}$. For $0<k \leq n$, the face maps of $\widehat{Z^{* x}}$ are $\hat{d}_{k}:=\sqcap T_{l}^{k-1} m_{l} T_{l}^{n-k} \sqcup$ while $\hat{d}_{0}:=\sqcap T_{l}^{n-1} m_{l} \sqcup \circ \hat{t}_{n}^{-1}$. We prove next that the operators $\tau_{*}$ are compatible with these face maps, i.e., for $0 \leq k \leq n$,

$$
\hat{d}_{k} \circ \tau_{n} \circ p \widehat{T}_{l}^{n+1} \hat{ப}=\tau_{n-1} \circ \hat{d}_{k} \circ p \hat{T}_{l}^{n+1} \hat{\mathrm{H}} .
$$

If $1 \leq k \leq n$, then proceeding as in the case of the para-cyclic operator one can see 
that the string representation of (6.10) is the following.
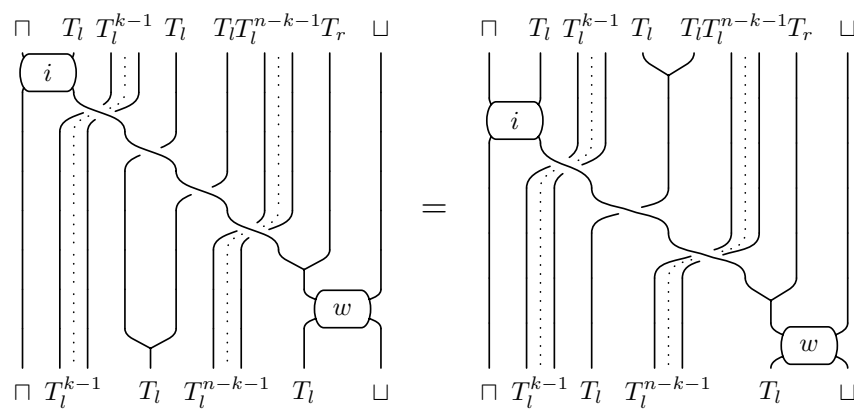

Visibly this equality holds, as the multiplication of a monad is a natural transformation and $\Phi$ is a distributive law. Finally, by the definition of para-cyclic objects, $\hat{d}_{0}=$ $\hat{d}_{n} \circ \hat{t}_{n}^{-1}$ and $\hat{d}_{0}=\hat{d}_{n} \circ \hat{t}_{n}^{-1}$. Thus, in view of relation (6.9) and of the fact that (6.10) holds for $k=n$, we conclude that (6.10) also holds for $k=0$. One proves in a similar way that the operators $\tau_{*}$ are compatible with the codegeneracy maps.

Our final task is to prove naturality of this isomorphism $\tau$. For an arbitrary morphism

$$
\zeta:=\left(G, q_{l}, q_{r}, \wedge, \pi, \vee, \omega\right):\left(T_{l}, T_{r}, \Phi, \sqcap, i, \sqcup, w\right) \rightarrow\left(T_{l}^{\prime}, T_{r}^{\prime}, \Phi^{\prime}, \Pi^{\prime}, i^{\prime}, \sqcup^{\prime}, w^{\prime}\right)
$$

in $\mathcal{A}_{c}^{\times}$, let $\hat{\zeta}:=\left(\widehat{G}, \hat{q}_{l}, \hat{q}_{r}, \wedge, \hat{\pi}, \vee, \widehat{\omega}\right)$ denote the corresponding morphism in $\mathscr{B}$. Since $p$ is a natural epimorphism, it is sufficient to prove that

$$
\wedge \tau_{*}^{\prime} \circ \widehat{\zeta}_{*} \circ p \widehat{T}_{l}^{n+1} \hat{\mathrm{U} \vee}=\hat{\zeta}_{*} \circ \tau_{*} \vee \circ p \widehat{T}_{l}^{n+1} \hat{\mathrm{U} \vee},
$$

where $\hat{\zeta}_{*}:=\mathcal{Z}_{*}^{\times}(\widehat{\zeta})$ (cf. Theorem 4.7), $\hat{\zeta}_{*}:=\widehat{\mathcal{Z}^{* \times}(\zeta)}$ (cf. Theorem 2.3), and $\tau_{*}$ and $\tau_{*}^{\prime}$ are morphisms constructed as in (6.8), corresponding to the domain and the codomain of $\zeta$, respectively. The string representations of both sides of (6.11) are given in the first and last terms of the following sequence of equations.
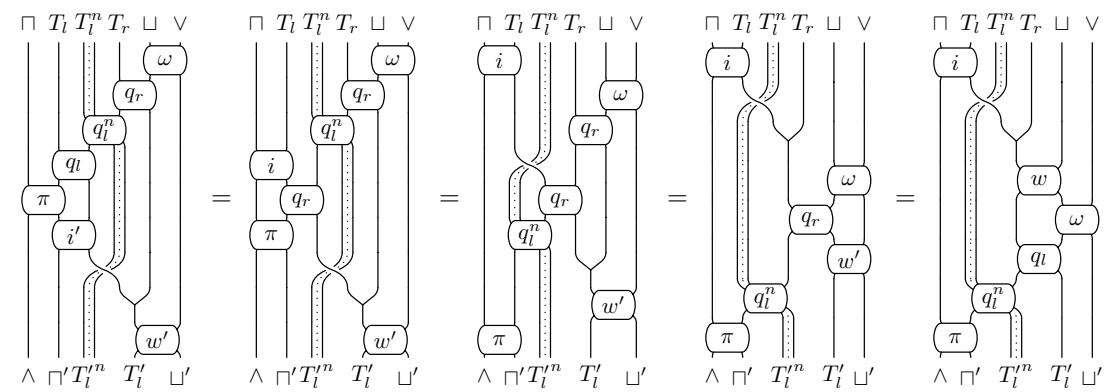

Recall that $q_{l}^{n}: T_{l}^{n} G \rightarrow G T_{l}^{\prime n}$ is defined inductively by $q_{l}^{1}=q_{l}$ and $q_{l}^{n+1}:=$ $q_{l} T_{l}^{\prime n} \circ T_{l} q_{l}^{n}$. The first identity follows by applying (1.5), while the second one is 
obtained by using repeatedly (1.2) and naturality. The third equation follows as $q_{r}$ is a morphism of monads and the last equality is a consequence of (1.6).

The statements about the functor $(\triangleq): \mathscr{B}_{e}^{\times} \rightarrow \mathcal{A}^{\times}$are proven symmetrically.

\section{Back to the examples}

In this section we return to the examples in Sections 3 and 5. We show that they all belong to $\mathcal{A}_{c}^{\times}$and $\mathscr{B}_{e}^{\times}$, respectively, whenever the occurring bialgebroids correspond to a Hopf algebroid with a bijective antipode and the occurring (co(ntra))modules are (co(ntra))modules of the Hopf algebroid (cf. Appendix). In light of Theorem 6.1, in this case we may apply the functor ${ }^{\Delta}$ to any of these examples. The examples in Section 3 and Section 5 turn out to be pairwise related via the functor ${ }^{\triangle}$.

Example 7.1. Let $H$ be a Hopf algebroid over base algebras $L$ and $R$, with a bijective antipode $S$, and $A$ be a left $H$-module algebra. This means that $A$ is a left module algebra of the constituent left bialgebroid $H_{L}$, so there is a corresponding object $\left(T_{l}, T_{r}, \Phi, \sqcap, i, \sqcup, w\right)$ of $\mathcal{A}$ in Example 3.1. Obviously, using the forgetful functor $F: H$-Comod $\rightarrow H_{L}$-Comod, we can construct another object

$$
\left(T_{l}, T_{r}, \Phi, \sqcap, i, \sqcup F, w F\right)
$$

of $\mathcal{A}$. We claim that (7.1) belongs to $\mathcal{A}^{\times}$(hence to $\mathcal{A}_{c}^{\times}$, since Mod- $k$ possesses coequalizers). Indeed, $\Phi$ and $i$ are obviously isomorphisms. The inverse of $w_{F M}$ is given, for a left $H$-comodule $M$ with coaction $m \mapsto m^{[-1]} \otimes_{R} m^{[0]}$ of the constituent right bialgebroid, by

$$
A \otimes_{L} M \rightarrow M \otimes_{L} A, \quad a \otimes_{L} m \mapsto m^{[0]} \otimes_{L} S^{-1}\left(m^{[-1]}\right) a .
$$

Thus we can apply to (7.1) the functor ${ }^{\wedge}$. The resulting object of $\mathcal{B}^{\times}$can be obtained from the object in Example 5.5, by composing on the right the left comodule functor $\sqcup:$ Comod- $H_{L} \rightarrow A$-Mod- $A$ in Example 5.5 with the forgetful functor Comod- $H \rightarrow$ Comod- $H_{L}$ and with the isomorphism $I_{S}: H$-Comod $\rightarrow$ Comod- $H$ induced by the bijective antipode $S$, cf. A.10.

Example 7.2. Let $H$ be a Hopf algebroid over base algebras $L$ and $R$, with a bijective antipode $S$, and $A$ be a right $H$-comodule algebra. Then $A$ is in particular a right comodule algebra of the constituent right bialgebroid. Hence there is a corresponding object of $\mathcal{A}$ as in Example 3.2. We claim that it belongs to $\mathcal{A}^{\times}$(hence to $\mathcal{A}_{c}^{\times}$). Indeed, $\Phi$ and $i$ are obviously isomorphisms. The inverse of $w_{N}$ is given, for any right $H$ module $N$, by

$$
A \otimes_{R} N \rightarrow N \otimes_{R} A, \quad a \otimes_{R} m \mapsto m S^{-1}\left(a_{[1]}\right) \otimes_{R} a_{[0]},
$$

where $a \mapsto a_{[0]} \otimes_{L} a_{[1]}$ denotes the coaction of the constituent left bialgebroid. Thus we can apply the functor ${ }^{\triangleleft}$. The resulting object of $\mathcal{B}^{\times}$can be obtained from the object 
in Example 5.6, by composing on the right the left comodule functor $\sqcup: H$-Mod $\equiv$ $H_{L}$-Mod $\rightarrow A$-Mod- $A$ in Example 5.6 with the isomorphism $I_{S^{-1}}^{-1}: \operatorname{Mod}-H \rightarrow$ $H$-Mod induced by the bijective antipode $S$, cf. A.9.

Example 7.3. Let $H$ be a Hopf algebroid over base algebras $L$ and $R$, with a bijective antipode $S$, and $C$ be a right $H$-module coring. This means that $C$ is a right module coring of the constituent right bialgebroid $H_{R}$, so there is a corresponding object $\left(T_{l}, T_{r}, \Phi, \sqcap, i, \sqcup, w\right)$ of $\mathcal{A}$ in Example 3.3. Using the forgetful functor $F: H$-Ctrmod $\rightarrow H_{R}$-Ctrmod, we can construct another object $\left(T_{l}, T_{r}, \Phi, \sqcap, i, \sqcup F, w F\right)$ of $\mathcal{A}$. We claim that the modified object belongs to $\mathcal{A}^{\times}$ (hence to $\mathcal{A}_{c}^{\times}$). Indeed, $\Phi$ and $i$ are obviously isomorphisms. The inverse of $w_{F Q}$ is given, for a left $H$-contramodule $Q$ with structure maps $\alpha_{L}: \operatorname{Hom}_{L,-}(H, Q) \rightarrow Q$ and $\alpha_{R}: \operatorname{Hom}_{R,-}(H, Q) \rightarrow Q$, by

$$
\operatorname{Hom}_{-, R}(C, Q) \rightarrow \operatorname{Hom}_{R,-}(C, Q), \quad f \mapsto\left(c \mapsto \alpha_{L}\left(f\left(c S^{-1}(-)\right)\right)\right) .
$$

Therefore we can apply the functor ${ }^{\triangle}$. The resulting object of $\mathscr{B}^{\times}$can be obtained from the object in Example 5.7, by composing on the right the left comodule functor $\sqcup$ : Ctrmod- $H_{R} \rightarrow C$-Ctrmod- $C$ in Example 5.7 with the forgetful functor Ctrmod- $H \rightarrow$ Ctrmod- $H_{R}$ and with the isomorphism $I_{S}: H$-Ctrmod $\rightarrow$ Ctrmod- $H$ induced by the bijective antipode $S$, cf. A.11.

Example 7.4. Let $H$ be a Hopf algebroid over base algebras $L$ and $R$, with a bijective antipode $S$, and $C$ be a left $H$-comodule coring. Then $C$ is in particular a left comodule coring of the constituent left bialgebroid. Hence there is a corresponding object of $\mathcal{A}$ as in Example 3.4. We claim that it belongs to $\mathcal{A}^{\times}$(hence to $\mathcal{A}_{c}^{\times}$). Indeed, $\Phi$ and $i$ are obviously isomorphisms. The inverse of $w_{N}$ is given, for any right $H$-module $N$, by

$$
\operatorname{Hom}_{-, L}(C, N) \rightarrow \operatorname{Hom}_{L,-}(C, N), \quad f \mapsto\left(c \mapsto f\left(c^{[0]}\right) S^{-1}\left(c^{[-1]}\right)\right),
$$

where $c \mapsto c^{[-1]} \otimes_{R} c^{[0]}$ denotes the coaction of the constituent right bialgebroid. Thus we can apply the functor ${ }^{\triangle}$. The resulting object of $\mathscr{B}^{\times}$can be obtained from the object in Example 5.8, by composing on the right the left comodule functor $\sqcup: H$-Mod $\equiv H_{R}$-Mod $\rightarrow C$-Comod- $C$ in Example 5.8 with the isomorphism $I_{S^{-1}}^{-1}:$ Mod- $H \rightarrow H$-Mod induced by the bijective antipode $S$, cf. A.9.

Example 7.5. Let $H$ be a Hopf algebroid over base algebras $L$ and $R$, with a bijective antipode $S$, and $C$ be a left $H$-comodule coring. Then $C$ is in particular a left comodule coring of the constituent left bialgebroid. Hence there is a corresponding object of $\mathscr{B}$ as in Example 5.1. We claim that it belongs to $\mathscr{B}^{\times}$(hence to $\mathscr{B}_{e}^{\times}$). Indeed, $\Psi$ and $i$ are obviously isomorphisms. The inverse of $w_{N}$ is given, for any left $H$-module $N$, by

$$
N \otimes_{L} C \rightarrow C \otimes_{L} N, \quad m \otimes_{L} c \mapsto c^{[0]} \otimes_{L} S^{-1}\left(c^{[-1]}\right) m,
$$


where $c \mapsto c^{[-1]} \otimes_{R} c^{[0]}$ denotes the coaction of the constituent right bialgebroid. Thus we can apply the functor ${ }^{\triangle}$. The resulting object of $\mathcal{A}^{\times}$can be obtained from the object in Example 3.5, by composing on the right the left comodule functor $\sqcup:$ Mod- $H \equiv \operatorname{Mod}-H_{R} \rightarrow C$-Comod- $C$ in Example 3.5 with the isomorphism $I_{S}: H$-Mod $\rightarrow$ Mod- $H$ induced by the bijective antipode $S$, cf. A.9.

Example 7.6. Let $H$ be a Hopf algebroid over base algebras $L$ and $R$, with a bijective antipode $S$, and $C$ be a right $H$-module coring. This means that $C$ is a right module coring of the constituent right bialgebroid $H_{R}$, so there is a corresponding object $\left(T_{l}, T_{r}, \Psi, \sqcap, i, \sqcup, w\right)$ of $\mathscr{B}$ in Example 5.2. Making use of the forgetful functor $F$ : Comod- $H \rightarrow$ Comod- $H_{R}$, we can construct another object $\left(T_{l}, T_{r}, \Psi, \sqcap, i, \sqcup F, w F\right)$ of $\mathscr{B}$. We claim that the modified object belongs to $\mathscr{B}^{\times}$ (hence to $\mathscr{B}_{e}^{\times}$). Indeed, $\Psi$ and $i$ are obviously isomorphisms. The inverse of $w_{F M}$ is given, for a right $H$-comodule $M$ with coaction $m \mapsto m_{[0]} \otimes_{L} m_{[1]}$ of the constituent left bialgebroid, by

$$
M \otimes_{R} C \rightarrow C \otimes_{R} M, \quad m \otimes_{R} c \mapsto c S^{-1}\left(m_{[1]}\right) \otimes_{R} m_{[0]} .
$$

Thus we can apply the functor ${ }^{\triangleleft}$. The resulting object of $\mathscr{A}^{\times}$can be obtained from the object in Example 3.6, by composing on the right the left comodule functor $\sqcup: H_{R}$-Comod $\rightarrow C$-Comod- $C$ in Example 3.6 with the forgetful functor

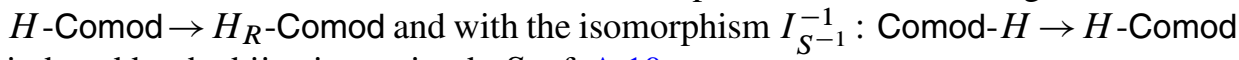
induced by the bijective antipode $S$, cf. A. 10 .

Example 7.7. Let $H$ be a Hopf algebroid over base algebras $L$ and $R$, with a bijective antipode $S$, and $A$ be a left $H$-module algebra. This means that $A$ is a left module algebra of the constituent left bialgebroid $H_{L}$, so there is a corresponding object $\left(T_{l}, T_{r}, \Psi, \sqcap, i, \sqcup, w\right)$ of $\mathscr{B}$ in Example 5.3. Making use of the forgetful functor $F$ : Ctrmod- $H \rightarrow$ Ctrmod- $H_{L}$, we can construct another object $\left(T_{l}, T_{r}, \Psi, \sqcap, i, \sqcup F, w F\right)$ of $\mathscr{B}$. We claim that the modified object belongs to $\mathscr{B}^{\times}$ (hence to $\mathscr{B}_{e}^{\times}$). Indeed, $\Psi$ and $i$ are obviously isomorphisms. The inverse of $w_{F Q}$ is given, for a right $H$-contramodule $\left(Q, \alpha_{L}, \alpha_{R}\right)$ by

$$
\operatorname{Hom}_{L,-}(A, Q) \rightarrow \operatorname{Hom}_{-, L}(A, Q), \quad g \mapsto\left(a \mapsto \alpha_{R}\left(g\left(S^{-1}(-) a\right)\right)\right) .
$$

Thus we can apply the functor ${ }^{\triangle}$. The resulting object of $\mathcal{A}^{\times}$can be obtained from the object in Example 3.7, by composing on the right the left comodule functor $\sqcup: H_{L}$-Ctrmod $\rightarrow A$-Mod- $A$ in Example 3.7 with the forgetful functor $H$-Ctrmod $\rightarrow$ $H_{L}$-Ctrmod and with the isomorphism $I_{S^{-1}}^{-1}:$ Ctrmod- $H \rightarrow H$-Ctrmod induced by the bijective antipode $S$, cf. A.11.

Example 7.8. Let $H$ be a Hopf algebroid over base algebras $L$ and $R$, with a bijective antipode $S$, and $A$ be a right $H$-comodule algebra. Then $A$ is in particular a right comodule algebra of the constituent right bialgebroid. Hence there is a corresponding 
object of $\mathscr{B}$ as in Example 5.4. We claim that it belongs to $\mathscr{B}^{\times}$(hence to $\mathscr{B}_{e}^{\times}$). Indeed, $\Psi$ and $i$ are obviously isomorphisms. The inverse of $w_{N}$ is given, for any left $H$-module $N$, by

$$
\operatorname{Hom}_{R,-}(A, N) \rightarrow \operatorname{Hom}_{-, R}(A, N), \quad g \mapsto\left(a \mapsto S^{-1}\left(a_{[1]}\right) g\left(a_{[0]}\right)\right),
$$

where $a \mapsto a_{[0]} \otimes_{L} a_{[1]}$ denotes the coaction of the constituent left bialgebroid. Thus we can apply the functor ${ }^{\triangle}$. The resulting object of $\mathcal{A}^{\times}$can be obtained from the object in Example 3.8, by composing on the right the left comodule functor $\sqcup$ : Mod$H \equiv \operatorname{Mod}-H_{L} \rightarrow A$-Mod- $A$ in Example 3.8 with the isomorphism $I_{S}: H$-Mod $\rightarrow$ Mod- $H$ induced by the bijective antipode $S$, cf. A.9.

\section{A. Appendix: Modules, comodules and contramodules of Hopf algebroids}

In this appendix we shortly review algebraic structures over non-commutative base algebras, which are used to construct the examples in the paper. For more information on them we refer to [2]. Structures as $R$-rings, $R$-corings, bialgebroids and Hopf algebroids below, generalize the notions of an algebra, a coalgebra, a bialgebra and a Hopf algebra over a commutative ring, respectively.

Throughout, let $k$ be a commutative, associative and unital ring. By an algebra $R$ we mean an associative and unital algebra over $k$. The enveloping algebra $R \otimes_{k} R^{\text {op }}$ is denoted by $R^{\mathrm{e}}$. We tacitly identify $R^{\mathrm{e}}$-modules with $R$-bimodules.

A.1. An $R$-ring is a monoid in the monoidal category of $R$-bimodules. In fact, an $R$-ring $A$ is equivalent to a $k$-algebra $A$, together with an algebra map $\iota: R \rightarrow A$. Denoting the multiplication in an $R$-ring $A$ by $\mu: A \otimes_{R} A \rightarrow A$, there is an induced monad

$$
\left((-) \otimes_{R} A,(-) \otimes_{R} \mu,(-) \otimes_{R} \iota\right)
$$

on the category Mod- $R$ of right $R$-modules. Algebras of this monad are equivalent to right modules of the $k$-algebra $A$. Symmetrically, algebras for the monad

$$
\left(A \otimes_{R}(-), \mu \otimes_{R}(-), \iota \otimes_{R}(-)\right)
$$

on the category $R$-Mod of left $R$-modules are equivalent to left modules of the $k$ algebra $A$. Note that the same formulae (A.1) and (A.2) define monads also on the category $R$-Mod- $R$ of $R$-bimodules, with respect to the $R$-actions

$$
r\left(p \otimes_{R} a\right) r^{\prime}=r p \otimes_{R} a r^{\prime} \quad \text { and } \quad r\left(a \otimes_{R} p\right) r^{\prime}=r a \otimes_{R} p r^{\prime}
$$

for $r, r^{\prime} \in R, a \in A, p \in P$ and any $R$-bimodule $P$.

A.2. For our considerations, $R^{\mathrm{e}}$-rings are of special interest. Note that an $R^{\mathrm{e}}$-ring is equivalent to an algebra $B$, together with algebra maps $s: R \rightarrow B$ and $t: R^{\text {op }} \rightarrow B$ 
such that $s(r) t\left(r^{\prime}\right)=t\left(r^{\prime}\right) s(r)$ for all $r, r^{\prime} \in R$. The maps $s$ and $t$ are known as the source and target maps, respectively. An immediate example of an $R^{\mathrm{e}}$-ring is the algebra $\operatorname{End}_{k}(R)$ of $k$-linear endomorphisms of $R$. It is an algebra via composition of endomorphisms and source and target maps are

$$
R \rightarrow \operatorname{End}_{k}(R), r \mapsto r(-), \quad R^{\mathrm{op}} \rightarrow \operatorname{End}_{k}(R), r \mapsto(-) r .
$$

Any $R^{\mathrm{e}}$-ring $B$ carries four commuting $R$-actions:

$$
r \triangleright b=s(r) b, \quad b \triangleleft r=t(r) b, \quad r \triangleright b=b t(r), \quad b \triangleleft r=b s(r) .
$$

In terms of these actions, the following construction can be performed. Take first the $R$-module tensor product

$$
B \otimes_{R} B:=B \otimes_{k} B /\left\{b \triangleleft r \otimes b^{\prime}-b \otimes r>b^{\prime} \text { for all } b, b^{\prime} \in B, r \in R\right\}
$$

and then the $k$-submodule

$B \times_{R} B:=\left\{\sum b_{i} \otimes_{R} b_{i}^{\prime} \in B \otimes_{R} B \mid \sum r \triangleright b_{i} \otimes_{R} b_{i}^{\prime}=\sum b_{i} \otimes_{R} b_{i}^{\prime} \triangleleft r\right.$ for all $\left.r \in R\right\}$.

$B \times{ }_{R} B$ is known as the Takeuchi product, and it is easily checked to be an $R^{\mathrm{e}}$-ring with factorwise multiplication and source and target maps

$$
R \rightarrow B \times_{R} B, r \mapsto s(r) \otimes_{R} 1_{B}, \quad R^{\text {op }} \rightarrow B \times_{R} B, r \mapsto 1_{B} \otimes_{R} t(r) .
$$

A.3. An $R$-coring is a comonoid in the monoidal category of $R$-bimodules. That is, an $R$-coring is an $R$-bimodule $C$ equipped with an $R$-bilinear coassociative comultiplication $\Delta: C \rightarrow C \otimes_{R} C$ possessing an $R$-bilinear counit $\epsilon: C \rightarrow R$. For the comultiplication we use the index notation $c \mapsto c_{\langle 1\rangle} \otimes_{R} c_{\langle 2\rangle}$, where implicit summation is understood.

Any $R$-coring $C$ induces a comonad

$$
\left((-) \otimes_{R} C,(-) \otimes_{R} \Delta,(-) \otimes_{R} \epsilon\right)
$$

on Mod- $R$. Coalgebras of this comonad are called right $C$-comodules. Explicitly, this means right $R$-modules $M$, equipped with a right $R$-linear coaction $M \rightarrow M \otimes_{R} C$, subject to coassociativity and counitality constraints. For a right coaction, the index notation $m \mapsto m_{[0]} \otimes_{R} m_{[1]}$ is used (with lower, or with upper indices), where implicit summation is understood. Symmetrically, coalgebras for the comonad

$$
\left(C \otimes_{R}(-), \Delta \otimes_{R}(-), \epsilon \otimes_{R}(-)\right)
$$

on $R$-Mod are called left $C$-comodules. For the coaction on a left $C$-comodule, we use the index notation $m \mapsto m_{[-1]} \otimes_{R} m_{[0]}$ (with lower or with upper indices), where implicit summation is understood. Morphisms of (right or left) $C$-comodules are morphisms of coalgebras for the appropriate comonad ((A.4) or (A.5)). That is, (right or left) $R$-module maps which are compatible with the $C$-coaction. 
Note that the same formulae (A.4) and (A.5) define comonads also on $R$-Mod- $R$, with respect to the $R$-bimodule structures as in (A.3).

To an $R$-coring $C$, one can associate also monads. The triple

$$
\left(\operatorname{Hom}_{-, R}(C,-), \operatorname{Hom}_{-, R}(\Delta,-), \operatorname{Hom}_{-, R}(\epsilon,-)\right)
$$

is a monad on Mod- $R$ (where we used standard hom-tensor identities to identify $\operatorname{Hom}_{-, R}\left(C \otimes_{R} C,-\right) \cong \operatorname{Hom}_{-, R}\left(C, \operatorname{Hom}_{-, R}(C,-)\right)$ and $\operatorname{Hom}_{-, R}(R,-) \cong$ Mod$R$ ). Algebras of this monad are called right $C$-contramodules, cf. [11], [3]. Symmetrically, left $C$-contramodules are algebras of the monad

$$
\left(\operatorname{Hom}_{R,-}(C,-), \operatorname{Hom}_{R,-}(\Delta,-), \operatorname{Hom}_{R,-}(\epsilon,-)\right)
$$

on $R$-Mod. Morphisms of (right or left) $C$-contramodules are morphisms of algebras for the appropriate monad ((A.6) or (A.7)). That is, (right or left) $R$-module maps which are compatible with the contramodule structure.

Note that the same formulae (A.6) and (A.7) define monads also on $R$-Mod- $R$, with respect to the $R$-bimodule structures

$$
\left(r f r^{\prime}\right)(c)=r f\left(r^{\prime} c\right) \text { and }\left(r g r^{\prime}\right)(c)=g(c r) r^{\prime}
$$

for $r, r^{\prime} \in R, c \in C, f \in \operatorname{Hom}_{-, R}(C, P), g \in \operatorname{Hom}_{R,-}(C, P)$ and any $R$-bimodule $P$.

A.4. A left $R$-bialgebroid [24], [20] is an $R^{\mathrm{e}}$-ring $(B, s, t)$ that also possesses an $R$-coring structure $(B, \boldsymbol{\downarrow}, \Delta, \epsilon)$ subject to the following compatibility axioms:

- The comultiplication $\Delta: B \rightarrow B \otimes_{R} B$ factorizes through $B \times_{R} B$;

- corestriction of $\Delta$ is a homomorphism of $R^{\mathrm{e}}$-rings $B \rightarrow B \times{ }_{R} B$;

- the map $B \rightarrow \operatorname{End}_{k}(R), b \mapsto \epsilon(b s(-))$ is a homomorphism of $R^{\mathrm{e}}$-rings.

Some equivalent forms of the definition can be found e.g. in [6]. The notion of a right $R$-bialgebroid is obtained symmetrically, by interchanging the roles of the $R$-actions $(\triangleright, \triangleleft)$ and $(\triangleright, \triangleleft)$ in an $R^{\mathrm{e}}$-ring, given by multiplication on the right, and on the left, respectively. For more details we refer to [15] or [2].

A.5. Modules of an $R$-bialgebroid $B$ are modules of the underlying $k$-algebra $B$. Since $B$ is an $R^{\mathrm{e}}$-ring, there is a forgetful functor from the category of (left or right) $B$-modules to the category of (left or right) $R^{\mathrm{e}}$-modules, equivalently, to the category of $R$-bimodules. By [22], Theorem 5.1, the category of left (resp. right) modules of a left (resp. right) bialgebroid is a monoidal category, with monoidal product given by the $R$-module tensor product. Left (resp. right) module algebras of a left (resp. right) bialgebroid $B$ are defined as monoids in the monoidal category of left (resp. right) $B$-modules. $B$-module algebras are thus in particular $R$-rings. By the same principle, left (resp. right) $B$-module corings are comonoids in the monoidal category of left (resp. right) $B$-modules. They are in particular $R$-corings. 
A.6. Comodules of a (left or right) $R$-bialgebroid are comodules of the constituent $R$ coring. As a consequence of the bialgebroid axioms, any right comodule $M$ of a right $R$-bialgebroid $B$ can be equipped also with a unique left $R$-action such that the range of the coaction $m \mapsto m^{[0]} \otimes_{R} m^{[1]}$ lies within the center of the $R$-bimodule $M \otimes_{R} B$. That is, for any $m \in M$ and $r \in R, r m^{[0]} \otimes_{R} m^{[1]}=m^{[0]} \otimes_{R} t(r) m^{[1]}$ (where $t: R^{\text {op }} \rightarrow B$ is the target map). This equips any $B$-comodule with an $R$-bimodule structure and the category of right $B$-comodules becomes monoidal with respect to the $R$-module tensor product (cf. [2], Theorem 3.18). In other words, there is a strict monoidal 'forgetful' functor from the category Comod- $B$ of right $B$-comodules to $R$ Mod- $R$. Symmetrically, also the category of left comodules of a right $R$-bialgebroid is monoidal, via $\otimes_{R^{\text {op }}}$. In the same way, categories of left and right comodules of a left $R$-bialgebroid are monoidal, with respect to the $R$-module tensor product and the $R^{\mathrm{op}}$-module tensor product, respectively. Left (resp. right) comodule algebras of a left or right bialgebroid $B$ are defined as monoids in the monoidal category of left (resp. right) $B$-comodules. $B$-comodule algebras are in particular $R$-rings or $R^{\text {op }}$ rings (depending on the monoidal product of the appropriate comodule category). By the same principle, left (resp. right) $B$-comodule corings are comonoids in the monoidal category of left (resp. right) $B$-comodules (hence they are $R$ or $R^{\text {op }}$-corings).

A.7. Contramodules of a (left or right) $R$-bialgebroid are contramodules of the constituent $R$-coring. As a consequence of the bialgebroid axioms, any left contramodule $\left(Q, \alpha: \operatorname{Hom}_{R,-}(B, Q) \rightarrow Q\right)$ of a right $R$-bialgebroid $B$ can be equipped also with a right $R$-action

$$
q r:=\alpha(\epsilon(s(r)-) q),
$$

for $q \in Q, r \in R$, such that $Q$ becomes an $R$-bimodule. This construction yields a 'forgetful' functor from the category $B$-Ctrmod of left $B$-contramodules to $R$-Mod$R$. Symmetrically, also right contramodules of a right $R$-bialgebroid and left and right contramodules of a left $R$-bialgebroid possess canonical $R$-bimodule structures. Note, however, that the category of contramodules of an arbitrary bialgebroid is not known to be monoidal.

A.8. A Hopf algebroid $H$ consists of a left bialgebroid structure $\left(H, s_{L}, t_{L}, \Delta_{L}, \epsilon_{L}\right)$ over a base algebra $L$, and a right bialgebroid structure $\left(H, s_{R}, t_{R}, \Delta_{R}, \epsilon_{R}\right)$ over a base algebra $R$, on the same $k$-algebra $H$, together with a $k$-module map $S: H \rightarrow H$, called the antipode. These structures are subject to the axioms

- $s_{R} \circ \epsilon_{R} \circ t_{L}=t_{L}, t_{R} \circ \epsilon_{R} \circ s_{L}=s_{L}, s_{L} \circ \epsilon_{L} \circ t_{R}=t_{R}, t_{L} \circ \epsilon_{L} \circ s_{R}=s_{R}$,

- $\left(\Delta_{R} \otimes_{L} H\right) \circ \Delta_{L}=\left(H \otimes_{R} \Delta_{L}\right) \circ \Delta_{R}$ and $\left(\Delta_{L} \otimes_{R} H\right) \circ \Delta_{R}=\left(H \otimes_{L} \Delta_{R}\right) \circ \Delta_{L}$,

- $S\left(t_{L}(l) h t_{R}(r)\right)=s_{R}(r) S(h) s_{L}(l)$ for all $h \in H, l \in L$ and $r \in R$,

- $\mu_{R} \circ\left(H \otimes_{R} S\right) \circ \Delta_{R}=s_{L} \circ \epsilon_{L}$ and $\mu_{L} \circ\left(S \otimes_{L} H\right) \circ \Delta_{L}=s_{R} \circ \epsilon_{R}$,

where $\mu_{R}: H \otimes_{R} H \rightarrow H$ denotes multiplication in the $R$-ring $s_{R}: R \rightarrow H$ and $\mu_{L}: H \otimes_{L} H \rightarrow H$ denotes multiplication in the $L$-ring $s_{L}: L \rightarrow H$. Note that 
the second axiom is meaningful because of the first axiom and the fourth axiom is meaningful because of the third one.

These axioms imply that the algebras $L$ and $R$ are anti-isomorphic, and the antipode is a bialgebroid morphism from the constituent left bialgebroid to the oppositecoopposite of the right bialgebroid, and also from the constituent right bialgebroid to the opposite-coopposite of the left bialgebroid.

A.9. Modules of a Hopf algebroid $H$ are by definition modules of the underlying $k$-algebra. In this way the category of (left or right) $H$-modules coincides with the (left or right) module category of any of the constituent bialgebroids. Hence both categories of left and right $H$-modules are monoidal. A (left or right) module algebra of a Hopf algebroid $H$ is defined as a monoid in the monoidal category of (left or right) $H$-modules. Similarly, a (left or right) module coring of a Hopf algebroid $H$ is defined as a comonoid in the monoidal category of (left or right) $H$-modules.

If the antipode $S$ of a Hopf algebroid $H$ is bijective, then it induces an isomorphism $I_{S}: H$-Mod $\rightarrow$ Mod- $H$ between the categories of left and right $H$-modules. This isomorphism takes a left $H$-module $N$ to $N$ as a right $H$-module with action $n \triangleleft h=$ $S^{-1}(h) n$. On the morphisms $I_{S}$ acts as the identity map. A similar isomorphism $I_{S^{-1}}: H$-Mod $\rightarrow$ Mod- $H$ is obtained by replacing $S$ by $S^{-1}$.

A.10. Right comodules of a Hopf algebroid $H$ over base algebras $L$ and $R$ are triples $\left(M, \varrho_{L}, \varrho_{R}\right)$, where $M$ is a right $L$-module and a right $R$-module, $\left(M, \varrho_{L}\right)$ is a right comodule of the constituent left bialgebroid, $\left(M, \varrho_{R}\right)$ is a right comodule of the constituent right bialgebroid such that both coactions are comodule maps for the other bialgebroid as well. That is, $\varrho_{L}$ is a right $R$-module map, $\varrho_{R}$ is a right $L$-module map and the compatibility conditions

$\left(M \otimes_{L} \Delta_{R}\right) \circ \varrho_{L}=\left(\varrho_{L} \otimes_{R} H\right) \circ \varrho_{R} \quad$ and $\quad\left(M \otimes_{R} \Delta_{L}\right) \circ \varrho_{R}=\left(\varrho_{R} \otimes_{L} H\right) \circ \varrho_{L}$

hold. It follows that the right $R$-, and $L$-actions on $M$ commute, i.e., $M$ is a right $R \otimes L$-module. Morphisms of $H$-comodules are defined as comodule maps for both constituent bialgebroids. Right comodules of a Hopf algebroid $H$ and their morphisms constitute the category Comod- $H$. The category $H$-Comod of left $H$ comodules is defined symmetrically.

The category of (left or right) comodules of a Hopf algebroid is monoidal and the forgetful functors to the comodule categories of the constituent bialgebroids are strict monoidal [2], Theorem 4.9. A (left or right) comodule algebra of a Hopf algebroid $H$ is defined as a monoid in the monoidal category of (left or right) $H$-comodules. Similarly, a (left or right) comodule coring of a Hopf algebroid $H$ is defined as a comonoid in the monoidal category of (left or right) $H$-comodules.

If the antipode $S$ of a Hopf algebroid $H$ is bijective, then it induces an isomorphism $I_{S}: H$-Comod $\rightarrow$ Comod- $H$. Take a left $H$-comodule $M$, with coaction $m \mapsto$ $m_{[-1]} \otimes_{L} m_{[0]}$ of the constituent left $L$-bialgebroid and coaction $m \mapsto m^{[-1]} \otimes_{R} m^{[0]}$ of the constituent right $R$-bialgebroid. The isomorphism $I_{S}$ takes it to $M$ as a 
right $H$-comodule, with right $R$, and $L$-actions induced by the algebra isomorphism $R \cong L^{\text {op }}$ and with coaction $m \mapsto m^{[0]} \otimes_{L} S^{-1}\left(m^{[-1]}\right)$ of the constituent left $L$ bialgebroid and coaction $m \mapsto m_{[0]} \otimes_{R} S^{-1}\left(m_{[-1]}\right)$ of the constituent right $R$ bialgebroid. On the morphisms $I_{S}$ acts as the identity map. A similar isomorphism $I_{S^{-1}}: H$-Comod $\rightarrow$ Comod- $H$ is obtained by replacing $S$ by $S^{-1}$.

A.11. Right contramodules of a Hopf algebroid $H$ over base algebras $L$ and $R$ are triples $\left(Q, \alpha_{L}, \alpha_{R}\right)$, where $Q$ is a right $L$-module and a right $R$-module, $\left(M, \alpha_{L}\right)$ is a right contramodule of the constituent left bialgebroid, $\left(M, \alpha_{R}\right)$ is a right contramodule of the constituent right bialgebroid such that both structure maps $\alpha_{L}$ and $\alpha_{R}$ are contramodule maps for the other bialgebroid as well. That is, $\alpha_{L}$ is a right $R$-module map, $\alpha_{R}$ is a right $L$-module map and the compatibility conditions

$$
\alpha_{L} \circ \operatorname{Hom}_{-, L}\left(H, \alpha_{R}\right)=\alpha_{R} \circ \operatorname{Hom}_{-, R}\left(\Delta_{L}, H\right)
$$

and

$$
\alpha_{R} \circ \operatorname{Hom}_{-, R}\left(H, \alpha_{L}\right)=\alpha_{L} \circ \operatorname{Hom}_{-, L}\left(\Delta_{R}, H\right)
$$

hold. It follows that the right $R$-, and $L$-actions on $Q$ commute, i.e., $Q$ is a right $R \otimes L$-module. Morphisms of $H$-contramodules are defined as contramodule maps for both constituent bialgebroids. Right contramodules of a Hopf algebroid $H$ and their morphisms constitute the category Ctrmod- $H$. The category $H$-Ctrmod of left $H$-contramodules is defined symmetrically.

If the antipode $S$ of a Hopf algebroid $H$ is bijective, then it induces an isomorphism $I_{S}: H$-Ctrmod $\rightarrow$ Ctrmod- $H$. Take a left $H$-contramodule $Q$, with structure map $\alpha_{L}: \operatorname{Hom}_{L,-}(H, Q) \rightarrow Q$ as a contramodule of the constituent left $L$-bialgebroid and structure map $\alpha_{R}: \operatorname{Hom}_{R,-}(H, Q) \rightarrow Q$ as a contramodule of the constituent right $R$-bialgebroid. The isomorphism $I_{S}$ takes it to $Q$ as a right $H$-contramodule, with right $R$, and $L$-actions induced by the algebra isomorphism $R \cong L^{\mathrm{op}}$ and with structure maps

$$
\operatorname{Hom}_{-, L}(H, Q) \rightarrow Q, \quad f \mapsto \alpha_{R}\left(f \circ S^{-1}\right),
$$

and

$$
\operatorname{Hom}_{-, R}(H, Q) \rightarrow Q, \quad g \mapsto \alpha_{L}\left(g \circ S^{-1}\right) .
$$

On the morphisms it acts as the identity map. A similar isomorphism

$$
I_{S^{-1}}: H \text {-Ctrmod } \rightarrow \text { Ctrmod- } H
$$

is obtained by replacing $S$ with $S^{-1}$.

Acknowledgments. The first named author was financially supported by the Hungarian Scientific Research Fund OTKA K 68195. The second named author was financially supported by CNCSIS, Contract 560/2009 (CNCSIS code ID_69). 


\section{References}

[1] J. Beck, Distributive laws. In Seminar on triples and Categorical homology theory (ETH, Zürich, 1966/67), Lecture Notes in Math. 80, Springer, Berlin 1969, 119-140. Zbl 0186.02902 MR 0241502

[2] G. Böhm, Hopf algebroids. In Handbook of algebra. Vol. 6, Elsevier/North-Holland, Amsterdam 2009, 173-235. Zbl 1220.16022 MR 2553659

[3] G. Böhm, T. Brzeziński, and R. Wisbauer, Monads and comonads on module categories. J. Algebra 322 (2009), 1719-1747. Zbl 1208.18003 MR 2543632

[4] G. Böhm and D. Ştefan, (Co)cyclic (co)homology of bialgebroids: an approach via (co)monads. Comm. Math. Phys. 282 (2008), 239-286. Zbl 1153.18004 MR 2415479

[5] T. Brzeziński, Hopf-cyclic homology with contramodule coefficients. In Quantum groups and noncommutative spaces, Aspects Math. E41, Vieweg, Wiesbaden 2011, 1-8. MR 2798432

[6] T. Brzeziński and G. Militaru, Bialgebroids, $\times_{A}$-bialgebras and duality. J. Algebra 251 (2002), 279-294. Zbl 1003.16033 MR 1900284

[7] E. Burroni, Algèbres non déterministiques et D-catégories. Cahiers Topologie Géom. Différentielle 14 (1973), 417-475. Zbl 0346.18007 MR 0357538

[8] A. Connes, Cohomologie cyclique et foncteurs $\mathrm{Ext}^{n}$. C. R. Acad. Sci. Paris Sér. I Math. 296 (1983), 953-958. Zbl 0534.18009 MR 777584

[9] A. Connes and H. Moscovici, Hopf algebras, cyclic cohomology and the transverse index theorem. Comm. Math. Phys. 198 (1998), 199-246. Zbl 0940.58005 MR 1657389

[10] A. Connes and H. Moscovici, Differentiable cyclic cohomology and Hopf algebraic structures in transverse geometry. In Essays on geometry and related topics, Vol. 1, Monogr. Enseign. Math. 38, Enseignement Math., Geneva 2001, 217-255. Zbl 1018.57013 MR 1929328

[11] S. Eilenberg and J. C. Moore, Foundations of relative homological algebra. Mem. Amer. Math. Soc. No. 55 (1965), 39. Zbl 0129.01101 MR 0178036

[12] P. M. Hajac, M. Khalkhali, B. Rangipour, and Y. Sommerhäuser, Hopf-cyclic homology and cohomology with coefficients. C. R. Math. Acad. Sci. Paris 338 (2004), 667-672. Zbl 1064.16006 MR 2065371

[13] P. Jara and D. Ştefan, Hopf-cyclic homology and relative cyclic homology of HopfGalois extensions. Proc. London Math. Soc. (3) 93 (2006), 138-174. Zbl 1158.16007 MR 2235945

[14] P. T. Johnstone, Adjoint lifting theorems for categories of algebras. Bull. London Math. Soc. 7 (1975), 294-297. Zbl 0315.18004 MR 0390018

[15] L. Kadison and K. Szlachányi, Bialgebroid actions on depth two extensions and duality. Adv. Math. 179 (2003), 75-121. Zbl 1049.16022 MR 2004729

[16] A. Kaygun, Bialgebra cyclic homology with coefficients. K-Theory 34 (2005), 151-194. Zbl 1114.16011 MR 2180111

[17] A. Kaygun, The universal Hopf-cyclic theory. J. Noncommut. Geom. 2 (2008), 333-351. Zbl 1189.16011 MR 2411421 
[18] M. Khalkhali and B. Rangipour, A note on cyclic duality and Hopf algebras. Comm. Algebra 33 (2005), 763-773. Zbl 1089.16011 MR 2128410

[19] J.-L. Loday, Cyclic homology. 2nd ed., Grundlehren Math. Wiss. 301, Springer-Verlag, Berlin 1998. Zbl 0885.18007 MR 1600246

[20] J.-H. Lu, Hopf algebroids and quantum groupoids. Internat. J. Math. 7 (1996), 47-70. Zbl 0884.17010 MR 1369905

[21] H.-E. Porst, On corings and comodules. Arch. Math. (Brno) 42 (2006), 419-425. Zbl 1152.16030 MR 2283022

[22] P. Schauenburg, Bialgebras over noncommutative rings and a structure theorem for Hopf bimodules. Appl. Categ. Structures 6 (1998), 193-222. Zbl 0908.16033 MR 1629385

[23] R. Street, The formal theory of monads. J. Pure Appl. Algebra 2 (1972), 149-168. Zbl 0241.18003 MR 0299653

[24] M. Takeuchi, Groups of algebras over $A \otimes \bar{A}$. J. Math. Soc. Japan 29 (1977), 459-492. Zbl 0349.16012 MR 0506407

[25] C. A. Weibel, An introduction to homological algebra. Cambridge Stud. Adv. Math. 38, Cambridge University Press, Cambridge 1994. Zbl 0797.18001 MR 1269324

Received October 24, 2009; revised July 21, 2010

G. Böhm, Institute for Particle and Nuclear Physics, Wigner Research Centre for Physics,

Budapest, 1525 Budapest 114, P.O. Box 49, Hungary

E-mail: bohm.gabriella@wigner.mta.hu

D. Ştefan, University of Bucharest, Faculty of Mathematics and Informatics, Bucharest, 14 Academiei Street, 010014, Romania

E-mail: drgstf@gmail.com 